\title{
METAL ABUNDANCES, RADIAL VELOCITIES, AND OTHER PHYSICAL CHARACTERISTICS FOR THE RR LYRAE STARS IN THE KEPLER FIELD*
}

\author{
James M. Nemec $^{1,2}$, Judith G. Cohen ${ }^{3}$, Vincenzo Ripepi ${ }^{4}$, Aliz Derekas $^{5,6}$, Pawel Moskalik ${ }^{7}$, \\ Branimir Sesar ${ }^{3}$, Merieme Chadid ${ }^{8}$, and Hans BruntT ${ }^{9}$ \\ ${ }^{1}$ Department of Physics \& Astronomy, Camosun College, Victoria, British Columbia, V8P 5J2, Canada; nemec@camosun.ca \\ 2 International Statistics \& Research Corp., Brentwood Bay, British Columbia, V8M 1R3, Canada; jmn@isr.bc.ca \\ ${ }^{3}$ Department of Physics and Astronomy, Caltech, Pasadena, CA, USA; jlc@astro.caltech.edu, bsesar@astro.caltech.edu \\ ${ }^{4}$ INAF-Osservatorio Astronomico di Capodimonte, Salita Moiariello 16, I-80131 Napoli, Italy; ripepi@na.astro.it \\ ${ }^{5}$ Konkoly Observatory, Research Centre for Astronomy and Earth Sciences, Hungarian Academy of Sciences, \\ H-1121 Budapest, Hungary; derekas@konkoly.hu \\ ${ }^{6}$ Sydney Institute for Astronomy, School of Physics, University of Sydney, Sydney, NSW 2006, Australia \\ ${ }^{7}$ Copernicus Astronomical Centre, ul.Bartycka 18, 00-716, Warsaw, Poland; pam@camk.edu.pl \\ ${ }^{8}$ Observatoire de la Côte d'Azur, Université de Nice, Sophia-Antipolis, UMR 6525, \\ Parc Valrose, F-06108 Nice Cedex 02, France; chadid@marseille.fr \\ ${ }^{9}$ Department of Physics \& Astronomy, Aarhus University, DK-8000 Aarhus C, Denmark; bruntt@phys.au.dk \\ Received 2013 March 25; accepted 2013 June 28; published 2013 August 6
}

\begin{abstract}
Spectroscopic iron-to-hydrogen ratios, radial velocities, atmospheric parameters, and new photometric analyses are presented for 41 RR Lyrae stars (and one probable high-amplitude $\delta$ Sct star) located in the field-of-view of the Kepler space telescope. Thirty-seven of the RR Lyrae stars are fundamental-mode pulsators (i.e., RRab stars) of which sixteen exhibit the Blazhko effect. Four of the stars are multiperiodic RRc pulsators oscillating primarily in the first-overtone mode. Spectroscopic $[\mathrm{Fe} / \mathrm{H}]$ values for the 34 stars for which we were able to derive estimates range from $-2.54 \pm 0.13$ (NR Lyr) to $-0.05 \pm 0.13 \mathrm{dex}$ (V784 Cyg), and for the 19 Kepler-field non-Blazhko stars studied by Nemec et al. the abundances agree will with their photometric $[\mathrm{Fe} / \mathrm{H}]$ values. Four non-Blazhko RR Lyrae stars that they identified as metal-rich (KIC 6100702, V2470 Cyg, V782 Cyg and V784 Cyg) are confirmed as such, and four additional stars (V839 Cyg, KIC 5520878, KIC 8832417, KIC 3868420) are also shown here to be metal-rich. Five of the non-Blazhko RRab stars are found to be more metal-rich than $[\mathrm{Fe} / \mathrm{H}] \sim-0.9$ dex while all of the 16 Blazhko stars are more metal-poor than this value. New $P-\phi_{31}^{s}-[\mathrm{Fe} / \mathrm{H}]$ relationships are derived based on $\sim 970$ days of quasi-continuous high-precision Q0-Q11 long- and short-cadence Kepler photometry. With the exception of some Blazhko stars, the spectroscopic and photometric $[\mathrm{Fe} / \mathrm{H}]$ values are in good agreement. Several stars with unique photometric characteristics are identified, including a Blazhko variable with the smallest known amplitude and frequency modulations (V838 Cyg).
\end{abstract}

Key words: stars: abundances - stars: variables: RR Lyrae

Online-only material: color figures

\section{INTRODUCTION}

A knowledge of the chemical compositions and kinematics of individual RR Lyrae stars is important for studying problems concerning stellar and galactic evolution and for understanding the evolution of stellar populations in our Galaxy and other nearby galaxies. With iron-to-hydrogen abundances, $[\mathrm{Fe} / \mathrm{H}]$, ranging from smaller than -2.5 dex to larger than 0.0 dex, RR Lyrae stars help to define the halo and old-disk stellar populations. They are ubiquitous, having been found in most globular clusters (see Clement et al. 2001), throughout the halo of our Galaxy (Le Borgne et al. 2012), in the Galactic bulge (Udalski et al. 1997; Alcock et al. 1998; Moskalik \& Poretti 2003; Collinge et al. 2006; Kunder \& Chaboyer 2008, 2009; Soszynski et al. 2011; Pietrukowicz et al. 2012), and in a growing number of nearby galaxies. Of particular interest are the large numbers found in the Large Magellanic Cloud (LMC;

\footnotetext{
* Based in part on observations made at the W.M. Keck Observatory, which is operated as a scientific partnership among the California Institute of Technology, the University of California and the National Aeronautics and Space Administration. The Keck Observatory was made possible by the generous financial support of the W.M. Keck Foundation. Also, based in part on observations obtained at the Canada-France-Hawaii Telescope (CFHT) which is operated by the National Research Council of Canada, the Institut National Des Sciences de l'Univers of the Centre National de la Recherche Scientifique of France, and the University of Hawaii.
}

Alcock et al. 1998, 2000; Gratton et al. 2004; Nemec et al. 2009; Soszynski et al. 2009), in the Small Magellanic Cloud (SMC; Soszynski et al. 2010), and in nearby dwarf spheroidal galaxies (see Garofalo et al. 2013 and references therein).

RR Lyrae stars are particularly valuable for stellar evolution studies because they are in an advanced (post-red giant branch, post-RGB) evolutionary stage and therefore serve to improve our understanding of mass loss, convection, rotation, and magnetic fields. One of their primary uses has been for distance estimation using an absolute magnitude, metallicity relation (see Sandage 1981). The linear form of this relation for field and cluster RR Lyr stars is usually written as $M_{V}(\mathrm{RR})=\alpha[\mathrm{Fe} / \mathrm{H}]+\beta$, where $\beta \sim 0.6$ and $\alpha$ ranges from $\sim 0.18-0.30$; however, there is strong evidence that the $M_{V}-[\mathrm{Fe} / \mathrm{H}]$ relationship is nonlinear over the range $[\mathrm{Fe} / \mathrm{H}] \sim-2.5$ to $\sim 0$ dex (Cassisi et al. 1999; Caputo et al. 2000; Rey et al. 2000; Bono et al. 2007). In either case it now seems clear that the more metal-rich RR Lyr stars have lower luminosities than their more metalpoor counterparts, at least for those stars near the zero-age horizontal branch (ZAHB) that are not evolved away from the ZAHB.

Another astrophysical problem of great interest is the unsolved Blazhko effect (see Kovács 2009; Buchler \& Kolláth 2011). Whatever the eventual explanation, the correct model will undoubtedly be subject to observational constraints such as are 
currently being provided by the Konkoly and Vienna Blazhko star surveys, and the high-precision, long-timeline photometry from the MOST, CoRoT, and Kepler space telescopes, as well as from ground-based follow-up surveys. These surveys suggest that a significant fraction of all RR Lyr stars, maybe as many as half, exhibit Blazhko characteristics; according to the Konkoly survey the fraction is at least $40 \%$ (Jurcsik et al. 2009c; Szeidl et al. 2012). For RR Lyr stars in the LMC the Blazhko effect is seen to be more frequent for stars with $[\mathrm{Fe} / \mathrm{H}]<-1.4$ dex than for more metal rich RR Lyrae stars (Smolec 2005). To test for a similar trend in our Galaxy, and for the derivation of distances and evolutionary states, requires accurate photometry and metal abundances.

In the $115 \mathrm{deg}^{2}$ field of view of NASA's Kepler space telescope $\sim 45$ RR Lyrae stars have been identified among stars down to $17.5 \mathrm{mag}$. Although this number is small compared with the sample sizes for the MACHO (Alcock et al. 2003, 2004), Optical Gravitational Lensing Experiment (OGLE; Soszynski et al. 2009, 2010), Sloan Digital Sky Survey (Sesar et al. 2010), All Sky Automated Survey (ASAS; Pojmanski 2002) and other modern large surveys, Kepler photometry provides the most precise and extensive set of photometry ever assembled for RR Lyr stars.

An early discovery made possible with Kepler data was the phenomenon of "period doubling," which manifests itself as alternating high and low amplitude pulsations (Szabó et al. 2010; Kolenberg et al. 2010; Buchler \& Kolláth 2011). The effect most probably is associated with the 9:2 resonance of the fundamental and overtone modes that destabilizes the fundamental-mode full amplitude pulsation (see Kolláth et al. 2011). Such period doubling usually is not detectable from the ground because day-night bias prevents observations of successive pulsation cycles. Period doubling is well-known in RV Tau and W Vir stars, and from models has been predicted in classical Cepheids (Moskalik \& Buchler 1991) and in BL Her stars (Buchler \& Moskalik 1992), the latter having been seen recently by Smolec et al. (2012).

The RR Lyrae stars in the Kepler field have been identified and studied previously by Kolenberg et al. (2010, 2011, hereafter K10, K11), Benkő et al. (2010, hereafter B10), Nemec et al. (2011, hereafter N11) and Guggenberger et al. (2012, hereafter G12). K10 published the first results based on early release (Q0,Q1) Kepler data, and carried out a detailed analysis of the Blazhko stars RR Lyrae and V783 Cyg. B10 presented a detailed analysis of 29 Kepler-field RR Lyrae stars based on the long cadence (LC) photometry from the first 138 days (Q0-Q2) of the Kepler observations, and found that almost half of the stars (14/29) show amplitude modulations. K11 studied RR Lyrae itself in great detail using Q1-Q2 LC data. The N11 study consisted of an extensive Fourier decomposition analysis (using Q0-Q5 Kepler photometry) of 19 non-Blazhko Kepler-field RRab stars. And most recently, G12 studied in detail the most extreme Blazhko star, V445 Lyr. The RRc stars, all of which are multiperiodic, are being studied in detail by Moskalik et al. (2012).

The primary goal of the present investigation was to derive spectroscopic $[\mathrm{Fe} / \mathrm{H}]_{\text {spec }}$ abundances (and the corresponding atmospheric parameters) for the Kepler-field RR Lyrae stars. Prior to the current investigation only photometric $[\mathrm{Fe} / \mathrm{H}]_{\text {phot }}$ values were known for the 19 non-Blazhko stars studied by N11; and neither spectroscopic nor photometric abundances had been measured for the RRc and Blazhko stars (a notable exception being RR Lyrae itself).
The reliability of the N11 photometric metallicities, especially for the four non-Blazhko stars identified as probable metal-rich old-disk stars, was of particular interest. Thus, a secondary goal was to confirm the Kepler-based $[\mathrm{Fe} / \mathrm{H}]_{\text {phot }}$ values for the non-Blazhko stars, and to use the longer time frame of the Q0-Q11 Kepler photometry (approaching 1000 days of quasicontinuous measurements) and the improved Kepler pipeline to derive $[\mathrm{Fe} / \mathrm{H}]_{\text {phot }}$ abundances for the Blazhko and RRc stars.

With $[\mathrm{Fe} / \mathrm{H}]_{\text {spec }}$ and $[\mathrm{Fe} / \mathrm{H}]_{\text {phot }}$ measurements in hand, a comparison of the metal abundances was possible. The good agreement found between the spectroscopic and photometric abundances suggests that high-precision photometry alone can be used to derive iron-to-hydrogen ratios, thereby providing a reliable means for the derivation of metallicities for stars too faint for high-dispersion spectroscopy (HDS).

In Section 2 the program stars are identified, and in Section 3 the Kepler photometry is described and used to derive photometric characteristics for the stars. In Section 4 the Canada-France-Hawaii Telescope (CFHT) and Keck high-dispersion spectra are described, from which radial velocities (RVs), spectroscopic metallicities, and correlations of the resulting atmospheric parameters are derived and discussed. The Kepler photometry and the new spectroscopic results are used in Section 5 to better establish the relationship between metal abundance and light curve morphology, in particular the RRab and RRc $P-\phi_{31}-[\mathrm{Fe} / \mathrm{H}]$ relations. Our results are summarized in Section 6.

\section{PROGRAM STARS}

The Kepler-field RR Lyrae stars studied here can be sorted into four distinct categories: (1) non-Blazhko RRab stars, (2) Blazhko RRab stars, (3) RRc stars, and (4) RRc/HADS? star. Table 1 contains some basic photometric characteristics for the stars. The first three columns contain the common star name (GCVS or other), the star number in the Kepler Input Catalog (KIC; see Brown et al. 2011), and the mean $K p$ magnitude given in the KIC. Columns 4 and 5 contain our best estimate of the pulsation period, $P_{\text {puls }}$, and a recent time of maximum light, $t_{0}$ (Barycentric Julian Date). Columns 6-8 contain the total amplitude, $A_{\text {tot }}$, the Fourier $A_{1}$ amplitude coefficient, and the Fourier $\phi_{31}^{s}$ phase parameter (defined as $\phi_{3}^{s}-3 \phi_{1}^{s}$, where the superscript " $s$ " indicates Fourier decomposition with a sine series), all derived from the $K p$ photometry (see Section 3). Column 9 contains the photometric metallicity, $[\mathrm{Fe} / \mathrm{H}]_{\text {phot }}$, derived in Section 5 using new bivariate linear regression analyses computed with the Kepler photometry (Section 3) and the new spectroscopic metallicities (Section 4).

In Table 1 the number of non-Blazhko stars is two greater than the 19 stars studied by N11: KIC 9658012, KIC 9717032, and V839 Cyg (KIC 10136603) are new additions, while V838 Cyg (KIC 10789273) has been moved to the Blazhko list. KIC 9658012 and KIC 9717032 were discovered during the course of the BOKS survey (Feldmeier et al. 2011); both stars were observed with Kepler for the first time in Q10, and neither star shows any evidence for amplitude modulations. A $g$-band light curve for KIC 9717032 is shown in Figure 17 of the Feldmeier paper. V839 Cyg is new and was discovered as a by-product of the ASAS-North survey (A. Pigulski et al. 2013, in preparation).

The Blazhko variables listed in Table 1 include three stars in addition to those in the B10 list: KIC 7257008 and KIC 9973633 (also discovered by A. Pigulski et al. 2013, in preparation) which show amplitude modulations in the Q10 and Q11 Kepler data; 
Table 1

Some Basic Photometric Characteristics for the Kepler-field RR Lyrae Stars

\begin{tabular}{|c|c|c|c|c|c|c|c|c|}
\hline Star & $\begin{array}{l}\mathrm{KIC} \\
(2)\end{array}$ & $\begin{array}{c}\langle K p\rangle \\
(\mathrm{mag}) \\
(3)\end{array}$ & $\begin{array}{c}P_{\text {puls }} \\
\text { (days) } \\
(4)\end{array}$ & $\begin{array}{c}t_{0}(\mathrm{BJD}) \\
2,400,000+ \\
(5)\end{array}$ & $\begin{array}{c}A_{\mathrm{tot}} \\
(\mathrm{mag}) \\
(6)\end{array}$ & $\begin{array}{c}A_{1} \\
(\mathrm{mag}) \\
(7)\end{array}$ & $\begin{array}{c}\phi_{31}^{s} \\
(\mathrm{rad}) \\
(8)\end{array}$ & $\begin{array}{c}{[\mathrm{Fe} / \mathrm{H}]_{\text {phot }}} \\
\text { (9) }\end{array}$ \\
\hline \multicolumn{9}{|c|}{ (a) 21 Non-Blazhko RRab-type stars } \\
\hline NR Lyr & 3733346 & 12.684 & 0.6820264 & 54964.7403 & 0.767 & 0.266 & 5.120 & $-2.51 \pm 0.06$ \\
\hline V715 Cyg & 3866709 & 16.265 & 0.47070609 & 54964.6037 & 0.988 & 0.338 & 4.901 & $-1.18 \pm 0.04$ \\
\hline V782 Cyg & 5299596 & 15.392 & 0.5236377 & 54964.5059 & 0.523 & 0.190 & 5.808 & $-0.30 \pm 0.04$ \\
\hline V784 Cyg & 6070714 & 15.37 & 0.5340941 & 54964.8067 & 0.634 & 0.234 & 6.084 & $-0.14 \pm 0.07$ \\
\hline KIC 6100702 & 6100702 & 13.458 & 0.4881457 & 54953.8399 & 0.575 & 0.209 & 5.747 & $-0.21 \pm 0.04$ \\
\hline NQ Lyr & 6763132 & 13.075 & 0.5877887 & 54954.0702 & 0.811 & 0.280 & 5.096 & $-1.81 \pm 0.03$ \\
\hline FN Lyr & 6936115 & 12.876 & 0.52739847 & 54953.2656 & 1.081 & 0.380 & 4.818 & $-1.90 \pm 0.04$ \\
\hline KIC 7021124 & 7021124 & 13.550 & 0.6224925 & 54965.6471 & 0.831 & 0.283 & 5.060 & $-2.18 \pm 0.04$ \\
\hline KIC 7030715 & 7030715 & 13.452 & 0.68361247 & 54953.8427 & 0.647 & 0.231 & 5.616 & $-1.28 \pm 0.05$ \\
\hline V349 Lyr & 7176080 & 17.433 & 0.5070740 & 54964.9588 & 0.988 & 0.346 & 4.850 & $-1.63 \pm 0.04$ \\
\hline V368 Lyr & 7742534 & 16.002 & 0.4564851 & 54964.7860 & 1.133 & 0.405 & 4.772 & $-1.29 \pm 0.05$ \\
\hline V1510 Cyg & 7988343 & 14.494 & 0.5811436 & 54964.6700 & 0.980 & 0.345 & 5.075 & $-1.80 \pm 0.03$ \\
\hline V346 Lyr & 8344381 & 16.421 & 0.5768288 & 54964.9231 & 0.964 & 0.330 & 5.052 & $-1.82 \pm 0.03$ \\
\hline V350 Lyr & 9508655 & 15.696 & 0.5942369 & 54964.7820 & 0.973 & 0.340 & 5.119 & $-1.81 \pm 0.03$ \\
\hline V894 Cyg & 9591503 & 13.293 & 0.5713866 & 54953.5624 & 1.105 & 0.377 & 5.067 & $-1.74 \pm 0.03$ \\
\hline KIC 9658012 & 9658012 & 16.001 & 0.533206 & 55779.9450 & 0.924 & 0.312 & 5.144 & $-1.28 \pm 0.02$ \\
\hline KIC 9717032 & 9717032 & 17.194 & 0.5569092 & 55779.8956 & 0.844 & 0.293 & 5.183 & $-1.38 \pm 0.02$ \\
\hline V2470 Cyg & 9947026 & 13.300 & 0.5485905 & 54953.7832 & 0.599 & 0.220 & 5.737 & $-0.47 \pm 0.03$ \\
\hline V1107 Cyg & 10136240 & 15.648 & 0.5657781 & 54964.7551 & 0.818 & 0.280 & 5.196 & $-1.42 \pm 0.02$ \\
\hline V839 Cyg & 10136603 & 14.066 & 0.4337747 & 55778.7060 & 0.793 & 0.273 & 5.600 & $-0.06 \pm 0.05$ \\
\hline AW Dra & 11802860 & 13.053 & 0.6872160 & 54954.2160 & 0.892 & 0.307 & 5.558 & $-1.42 \pm 0.05$ \\
\hline \multicolumn{9}{|c|}{ (b) 16 Blazhko RRab-type stars } \\
\hline V2178 Cyg & 3864443 & 15.593 & 0.4869538 & 54976.3672 & 0.749 & 0.305 & 4.895 & $-1.35 \pm 0.03$ \\
\hline V808 Cyg & 4484128 & 15.363 & 0.5478642 & 54970.2834 & 0.879 & 0.298 & 5.254 & $-1.18 \pm 0.03$ \\
\hline V783 Cyg & 5559631 & 14.643 & 0.62070001 & 54975.5439 & 0.799 & 0.271 & 5.517 & $-1.15 \pm 0.03$ \\
\hline V354 Lyr & 6183128 & 16.260 & 0.561691 & 55245.1590 & 0.815 & 0.303 & 5.191 & $-1.40 \pm 0.02$ \\
\hline V445 Lyr & 6186029 & 17.401 & 0.5131158 & 55160.5957 & 0.591 & 0.255 & 5.198 & $-1.02 \pm 0.03$ \\
\hline RR Lyrae & 7198959 & 7.862 & 0.566788 & 55278.2263 & 0.717 & 0.255 & 5.307 & $-1.21 \pm 0.03$ \\
\hline KIC 7257008 & 7257008 & 16.542 & 0.51177516 & 55758.5859 & 0.818 & 0.290 & 5.191 & $-1.02 \pm 0.03$ \\
\hline V355 Lyr & 7505345 & 14.080 & 0.4737027 & 55124.7072 & 0.955 & 0.373 & 4.928 & $-1.16 \pm 0.04$ \\
\hline V450 Lyr & 7671081 & 16.653 & 0.5046123 & 54996.3226 & 0.850 & 0.335 & 4.976 & $-1.35 \pm 0.03$ \\
\hline V353 Lyr & 9001926 & 16.914 & 0.5568016 & 55082.6820 & 0.834 & 0.293 & 5.123 & $-1.50 \pm 0.02$ \\
\hline V366 Lyr & 9578833 & 16.537 & 0.5270283 & 55326.1915 & 0.856 & 0.308 & 5.167 & $-1.18 \pm 0.02$ \\
\hline V360 Lyr & 9697825 & 16.001 & 0.5575765 & 54988.9332 & 0.647 & 0.257 & 5.065 & $-1.63 \pm 0.02$ \\
\hline KIC 9973633 & 9973633 & 16.999 & 0.51075 & 55780.3655 & 0.848 & 0.293 & 4.122 & $-1.17 \pm 0.03$ \\
\hline V838 Cyg & 10789273 & 13.770 & 0.48027971 & 55807.9302 & 1.100 & 0.390 & 4.857 & $-1.36 \pm 0.04$ \\
\hline KIC 11125706 & 11125706 & 11.367 & 0.6132200 & 54981.0658 & 0.471 & 0.180 & 5.824 & $-0.61 \pm 0.05$ \\
\hline V1104 Cyg & 12155928 & 15.033 & 0.43638507 & 55120.8363 & 1.103 & 0.394 & 4.861 & $-0.93 \pm 0.05$ \\
\hline \multicolumn{9}{|c|}{ (c) 4 RRc-type stars } \\
\hline KIC 4064484 & 4064484 & 14.641 & 0.33700953 & 55552.1567 & 0.377 & 0.193 & 0.494 & $-1.59 \pm 0.02$ \\
\hline KIC 5520878 & 5520878 & 14.214 & 0.26917082 & 55800.0883 & 0.325 & 0.164 & 0.908 & $-0.36 \pm 0.06$ \\
\hline KIC 8832417 & 8832417 & 13.051 & 0.2485492 & 54964.6391 & 0.281 & 0.141 & 0.727 & $-0.20 \pm 0.07$ \\
\hline KIC 9453114 & 9453114 & 13.419 & 0.3660236 & 55740.6420 & 0.414 & 0.209 & 0.865 & $-1.70 \pm 0.02$ \\
\hline
\end{tabular}

(d) High-amplitude Delta Scuti (HADS) star?

\begin{tabular}{lllllllllll}
\hline KIC 3868420 & 3868420 & 10.110 & 0.2082275 & 54960.5192 & 0.171 & 0.083 & 4.109 & $\ldots$
\end{tabular}

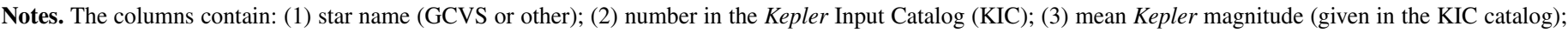

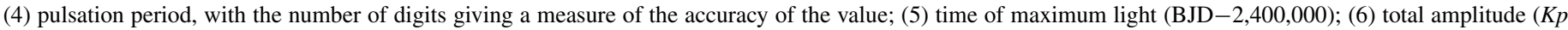

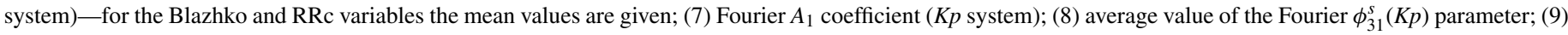

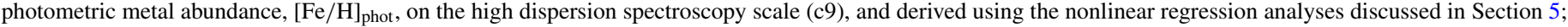
the uncertainties are standard errors of the regression fit.

and V838 Cyg which was previously thought to be a nonBlazhko variable but now appears to be the lowest-amplitude Blazhko star yet discovered (see below). Wils et al. (2006) listed NR Lyr (KIC 3733346) as a Blazhko star with an uncertain modulation period of 27 days; however, our analysis of the Q1-Q13 LC data (51,883 data points) and the Q11.1 short cadence (SC) data (45,512 data points) showed no evidence for amplitude modulations, thus supporting the B10 and N11 conclusion that NR Lyr is a non-Blazhko RRab variable. The faint star V349 Lyr (KIC 7176080) is in the B10 Blazhko list but was classified by N11 as a probable non-Blazhko star; our analysis of the Q1-Q13 LC data (51,877 data points) and the 
Q9 SC data (51,878 data points) again shows no evidence for amplitude modulations.

To date only four bona fide c-type RR Lyrae (RRc) stars have been identified in the Kepler field. Two were observed at CFHT and two at Keck: the two shorter period stars are metal rich, and the two longer period stars are metal poor (see Sections 4 and 5 below). All four stars have been found to be multiperiodic and are being studied in detail by Moskalik et al. (2012) and P. Moskalik et al. (2013, in preparation). Owing to the challenges of distinguishing RRc stars from high-amplitude $\delta$ Sct (HADS) stars and some close eclipsing binaries, all of which have similar light curves, the RRc section of Table 1 probably is more incomplete than the RRab sections. KIC 10063343 $(K p=13.16)$ has a pulsation period appropriate for an RRc star, $P_{\text {puls }}=0.332764$ days, but the variable light curve and Fourier parameters raise serious doubts that it is an RR Lyrae star. Other candidate RRc stars are being sought by K. Kinemuchi et al. (2013, in preparation).

The relatively bright star KIC $3868420(K p=10.11)$ is of particular interest. The early Kepler photometry suggested that it might be an RRc star, and as such a single spectrum was taken at CFHT. Analysis of this spectrum revealed it to be a hot, high surface gravity star with spectroscopic $[\mathrm{Fe} / \mathrm{H}]=$ $-0.32 \pm 0.13 \mathrm{dex}$; however, with a dominant period of only 0.208 days, a light curve that is more symmetric and more sharply peaked than the light curves of the RRc stars, and a relatively small RV, it more probably is a HADS star.

Three additional RRab stars recently have been identified in the Kepler field, in the directions of KIC 3448777, KIC 4917786, and KIC 7295372. All have $P_{\text {puls }} \sim 0.5$ days, low amplitudes resulting from "crowding" by brighter nearby stars, ${ }^{10}$ and unknown metal abundances. These newly discovered RR Lyr stars continue to be observed by Kepler.

\section{KEPLER PHOTOMETRY}

An unprecedented amount of high-precision Kepler photometry is currently available for deriving the photometric characteristics (including photometric metal abundances) for the majority of the stars in Table 1. Most of the program RR Lyr stars have been observed every quarter since the first Kepler flux measurements were made in 2009 May. Up to and including Q11 a typical RRab star with a pulsation period of 0.5 day had over $3.5 \mathrm{yr}$ of quasi-continuous high-precision photometric data. LC observations (i.e., 29.4 minute flux integrations) over this $3.5 \mathrm{yr}$ interval amount to $\sim 350,000$ data points spread over 2500 pulsation cycles, with $\sim 24$ brightness measurements per cycle. For most of the stars the CFHT and Keck spectra had simultaneously acquired Kepler photometry. The CFHT spectroscopic observations were made during the 6th and 7th quarters (Q6,Q7) of the Kepler observations, and the Keck spectra were acquired during the first month of the 10th quarter (Q10.1).

Five of the RR Lyr stars in Table 1 were not observed with Kepler prior to the start of Q10: KIC 7257008, KIC 9658012, KIC 9717032, KIC 9973633, and V839 Cyg. Fortunately, the Keck observations for the last four stars were made in 2011 August, which occurs in Q10.1 of the Kepler observations. KIC $7257008(K p=16.54)$, KIC $7021124(K p=$ $13.55)$, and V349 Lyr $(K p=17.43)$ have yet to be observed spectroscopically.

${ }^{10}$ The "crowding" here is determined by the $3.98 \mathrm{arcsec}^{\mathrm{ax}} \mathrm{el}^{-1}$ image scale of the CCD chips on the Kepler telescope.

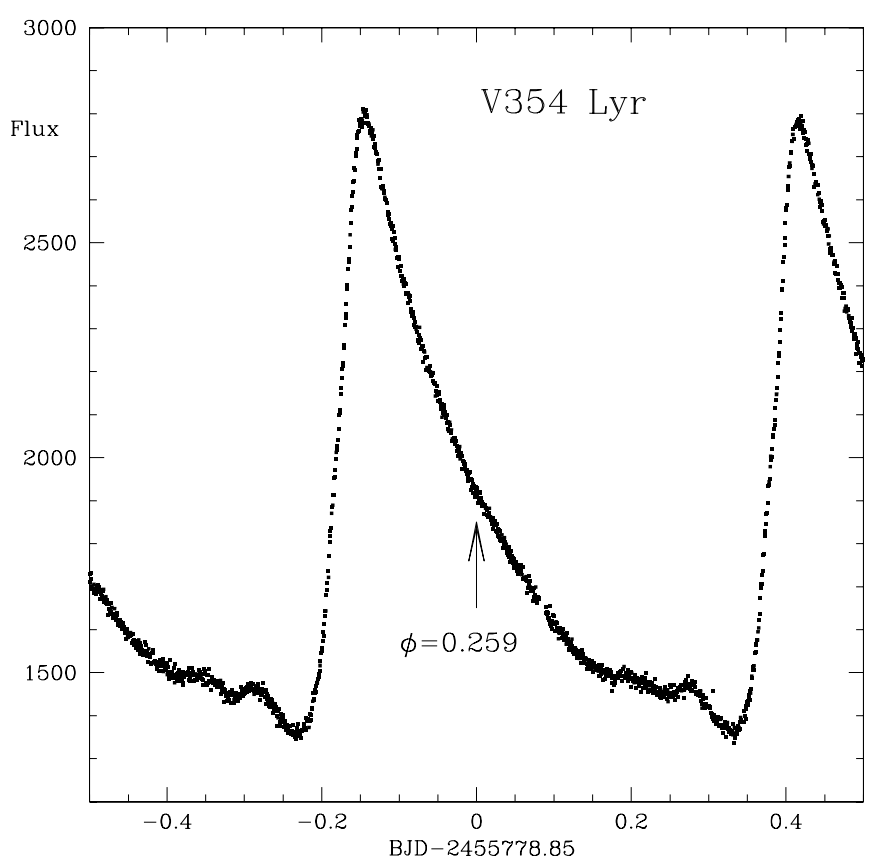

Figure 1. A typical Kepler light curve, in this case for V354 Lyr (KIC 6183128), a Blazhko RRab star with $P_{\text {puls }}=0.561691 \pm 0.000001$ days and $P_{\mathrm{BL}}=$ $723 \pm 12$ days. The data plotted are a one-day segment of short cadence flux measurements made simultaneously with three Keck-I $10 \mathrm{~m}$ spectra. The abscissa is time (Barycentric Julian Date), starting 0.5 day before and ending 0.5 day after the mid-time of the spectra; and the ordinate is the Kepler raw flux (counts $\mathrm{s}^{-1}$ ). The pulsation phase at the mid-time of the spectroscopic observations is indicated by a labeled arrow.

In addition to the LC data most of the program stars also have been observed at SC (i.e., a flux measurement every 1 minute) for at least one or two quarters. The available SC data for these stars usually amounts to almost half a million observation points per star. RR Lyrae itself has been observed at SC every quarter since Q5, the total number of data points being just under $9 \times 10^{5}$.

A typical RRab star with SC data has $\sim 720$ brightness measurements per pulsation cycle, which is sufficient for defining extremely well the shape of the light curve (as illustrated in Figure 1), and for detecting and defining from precise Fourier parameters the smallest amplitude and frequency modulations.

The task of processing the Kepler raw flux data has been discussed by Koch et al. (2010), Jenkins et al. (2010), and Gilliland et al. (2010). Of particular concern was the detrending of data within quarters, the "stitching together" of the data from the different quarters, and the merging of LC and SC observations. The reduction methods (transforming the raw flux counts to $K p$ magnitudes) that were used here were similar to those described in N11. To achieve the highest levels of photometric precision multiple detrending passes were found to be necessary to reduce the residuals about the non-Blazhko light curves to $\sim 0.2 \mathrm{mmag}$.

\subsection{Pulsation Periods, Amplitudes, and Phases}

Pulsation periods, $P_{\text {puls }}$, times of maximum light, $t_{0}$, and total amplitudes on the Kepler photometric system, $A_{\text {tot }}$, are given for the program RR Lyrae stars in Columns 4-6 of Table 1. All values are newly derived based on analyses of the available Kepler Q0-Q11 LC and SC data, which in most cases comprises almost 1000 days of quasi-continuous high-precision photometry. 

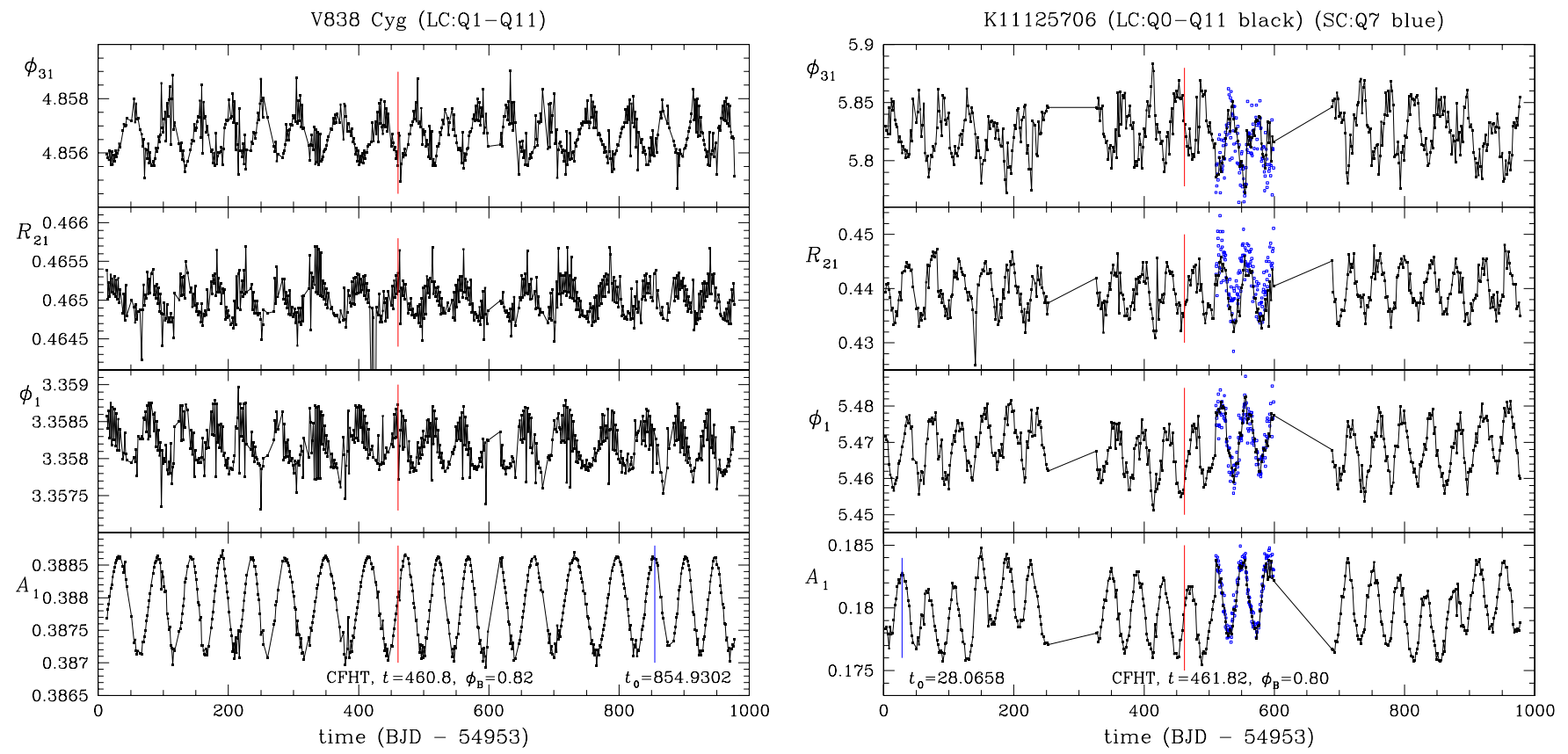

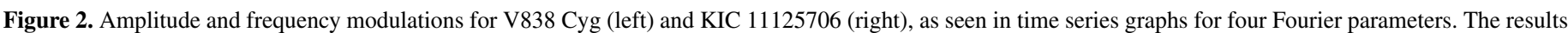

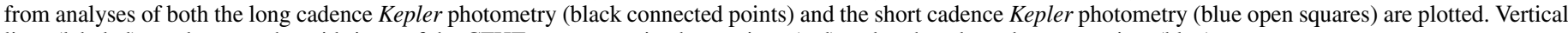
lines (labeled) are drawn at the mid-times of the CFHT spectroscopic observations (red) and at the adopted $t_{0}$ zero points (blue).

(A color version of this figure is available in the online journal.)

The $P_{\text {puls }}$ are mean values of the dominant oscillation frequencies, ignoring the secondary periods that are present in some non-Blazhko stars (V350 Lyr, KIC 7021124) and in all the Blazhko stars, ignoring changing periods, and averaging over many modulation cycles for the Blazhko stars. The periods were computed with the "Period04" package of Lenz \& Breger (2005) using the Monte Carlo mode for error estimation, with Stellingwerf's (1978) "PDM2" program ${ }^{11}$ updated to deal with rich data sets, and with the period-finding program of Kolaczkowski (see Moskalik \& Kołaczkowski 2009).

The accuracy of the pulsation periods reported in Table 1 is $\sim$ few units in the last decimal place given, with the periods for the Blazhko and RRc stars tending to be less accurate than those for the non-Blazhko stars. The uncertainties depend on several factors: the particular data set (or subset) that was analyzed, the search method, the constancy of the primary pulsation period, and whether or not amplitude or frequency modulations are present. In general the periods agree well with those given by B10 for 15 non-Blazhko and 14 Blazhko stars, and with the more precise values derived by N11 for 19 non-Blazhko stars. Since the new periods were computed with longer time baselines than these earlier studies, and are based on both LC and SC data, they are the best available values yet reported for these stars. In general, the accuracy of the $P_{\text {puls }}$ values is more than sufficient for estimating the phases at which the spectroscopic observations were made.

The $A_{\text {tot }}$ in Table 1 are the trough-to-peak $K p$ amplitudes. For the non-Blazhko stars they are time invariant values obtained by inspection of the complete light curve and by averaging $A_{\text {tot }}$ calculated from individual light curves for successive time segments (see Figure 2). For the Blazhko variables and the four RRc stars, all of which are multiperiodic and show amplitude variations, $A_{\text {tot }}$ is the time-averaged value. According to the transformation given in Equation (2) of N11 the $A_{\mathrm{tot}}(K p)$ are

\footnotetext{
11 PDM2 is freely available at http://www.stellingwerf.com.
}

$\sim 0.14$ mag smaller than the $A_{\text {tot }}(V)$, where $V$ is for the Johnson $U B V$ system.

The zero points $t_{0}$ were estimated from the mean light curves and correspond to times of zero-slope at maximum light. For the non-Blazhko stars they were usually chosen to approximately coincide with the time of the first Kepler observations (i.e., just after BJD-2,454,953), and for the Blazhko variables the $t_{0}$ generally coincide with a time of maximum light occurring during a period of maximum amplitude. Thus, $t_{0}$ serves as the zero point for both the pulsation phase, $\phi_{\text {puls }}$, defined to be the fractional part of $\left(t-t_{0}\right) / P_{\text {puls }}$, and for the Blazhko phase, $\phi_{\mathrm{BL}}$, defined to be the fractional part of $\left(t-t_{0}\right) / P_{\mathrm{BL}}$.

\subsection{Blazhko Period and Amplitude Modulations}

The most noticeable signature of the Blazhko effect is variation in the light curve shape, in particular amplitude modulations, over time scales ranging from several days to several years. The amplitude that is usually considered is the total amplitude (i.e., from trough to peak) through the $V$ or $B$ filter, but may also be one of the Fourier amplitude coefficients. In the absence of $B V$ photometry for most of the Kepler-field RR Lyr stars we focus in this paper on the $A_{\text {tot }}$ and $A_{1}$ amplitudes derived from the $K p$ photometry. The $A_{1}$ values are from Fourier decompositions of the $K p$ data performed using the following sine-series sum:

$$
m(t)=A_{0}+\sum_{k=1}^{N} A_{k} \sin \left(k 2 \pi t / P+\phi_{k}\right),
$$

where $m(t)$ is the apparent $K p$ magnitude as a function of time, $A_{0}$ is the mean $K p$ magnitude (assumed to be the value given in the KIC catalog), $N$ is the adopted number of terms in the Fourier series, the $A_{k}$ and $\phi_{k}$ are the Fourier amplitudes and phases, and $P$ is the pulsation period. 
Table 2

Amplitude and Period (Phase) Modulations of 16 Blazhko Stars in the Kepler Field

\begin{tabular}{|c|c|c|c|c|c|}
\hline Star & $\begin{array}{c}\Delta A_{\text {tot }} \\
(\mathrm{mag}) \\
(2)\end{array}$ & $\begin{array}{c}\Delta P_{\text {puls }} \\
\text { (days) } \\
\text { (3) }\end{array}$ & $\begin{array}{c}\Delta A_{1} \\
(\mathrm{mag}) \\
(4)\end{array}$ & $\begin{array}{c}\Delta \phi_{1} \\
(\mathrm{rad}) \\
(5)\end{array}$ & $\begin{array}{c}\Delta \phi_{31}^{s} \\
(\mathrm{rad}) \\
(6)\end{array}$ \\
\hline V445 Lyr & $0.80(0.20-1.00)$ & $0.013(0.509-0.522)$ & $0.380(0.018-0.398)$ & $3.12(1.48-4.60)$ & $6.28(0.00-6.28)$ \\
\hline V2178 Cyg & $0.84(0.30-1.14)$ & $0.0014(0.4859-73)$ & $0.279(0.162-0.441)$ & $1.09(3.91-5.00)$ & $3.55(3.41-6.96)$ \\
\hline V450 Lyr & $0.56(0.57-1.13)$ & $0.0007(0.5042-49)$ & $0.240(0.207-0.447)$ & $0.44(4.74-5.18)$ & $1.12(4.53-5.65)$ \\
\hline KIC 7257008 & $0.68(0.44-1.12)$ & $0.0030(0.5105-35)$ & $0.211(0.179-0.390)$ & $0.60(3.00-3.60)$ & $1.05(4.77-5.82)$ \\
\hline V354 Lyr & $0.60(0.49-1.09)$ & $0.0003(0.5616-19)$ & $>0.20(<0.19-0.39)$ & $1.26(2.51-3.77)$ & $0.70(4.85-5.55)$ \\
\hline V360 Lyr & $0.21(0.54-0.75)$ & $0.0010(0.5570-80)$ & $0.162(0.176-0.338)$ & $0.37(0.99-1.36)$ & $0.82(4.78-5.60)$ \\
\hline V808 Cyg & $0.35(0.71-1.06)$ & $0.0020(0.5467-87)$ & $0.130(0.237-0.367)$ & $0.67(0.28-0.95)$ & $0.64(4.96-5.60)$ \\
\hline RR Lyrae & $0.23(0.59-0.82)$ & $0.0024(0.5657-81)$ & $0.127(0.186-0.313)$ & $0.65(4.77-5.42)$ & $1.21(4.85-6.06)$ \\
\hline V366 Lyr & $0.18(0.77-0.95)$ & $0.0003(0.5269-72)$ & $0.127(0.248-0.375)$ & $0.14(1.21-1.35)$ & $0.98(4.75-5.73)$ \\
\hline KIC 9973633 & $0.39(0.61-1.00)$ & $0.0009(0.5105-14)$ & $0.119(0.225-0.344)$ & $0.22(4.00-4.22)$ & $0.78(4.80-5.58)$ \\
\hline V355 Lyr & $0.15(0.87-1.02)$ & $0.0008(0.4733-41)$ & $0.110(0.313-0.423)$ & $0.15(1.05-1.20)$ & $0.62(4.67-5.29)$ \\
\hline V353 Lyr & $0.24(0.71-0.95)$ & $0.0004(0.5566-70)$ & $0.082(0.250-0.332)$ & $0.16(0.59-0.75)$ & $0.24(5.01-5.25)$ \\
\hline V1104 Cyg & $0.17(1.03-1.20)$ & $0.0003(0.4363-66)$ & $0.062(0.365-0.427)$ & $0.070(0.165-0.235)$ & $0.16(4.78-4.94)$ \\
\hline V783 Cyg & $0.08(0.76-0.84)$ & $0.0005(0.6204-09)$ & $0.033(0.253-0.286)$ & $0.08(1.82-1.90)$ & $0.14(5.44-5.58)$ \\
\hline KIC 11125706 & $0.03(0.45-0.48)$ & $0.0004(0.6128-32)$ & $0.008(0.176-0.184)$ & $0.031(5.452-5.483)$ & $0.112(5.772-5.884)$ \\
\hline V838 Cyg & $0.02(1.09-1.11)$ & $0.0002(0.4801-03)$ & $0.0016(0.3871-87)$ & $0.0010(3.3577-87)$ & $0.003(4.855-4.858)$ \\
\hline
\end{tabular}

Notes. The columns contain: (1) star name; (2) range of total amplitude ( $K p$-system), with minimum and maximum values in parentheses; (3) range of the pulsation period (min and max in parentheses); (4) range of the Fourier $A_{1}$ amplitude coefficient (min and max in parentheses ) - note that $\Delta A_{1}$ here is the $A_{1}$ range, which is not the same as the $\Delta A_{1}$ defined by $\mathrm{B} 10$; (5) range of the first Fourier phase coefficient, $\phi_{1}$ (min and max in parentheses); (6) range of the Fourier phase-difference parameter $\phi_{31}^{s}$ ( $\min$ and max in parentheses).

Blazhko stars are also known to exhibit period (frequency, or phase) modulations, although usually these are less noticeable than the amplitude modulations. Frequency modulations were detected previously in the Kepler-field Blazhko stars studied by B10, in RR Lyrae by K10 and K11, and in V445 Lyr by G12. The 16 Kepler-field Blazhko stars studied here all exhibit both amplitude and frequency modulations.

While the detection of amplitude and frequency modulations is usually straightforward, the physical mechanism responsible remains unknown, and Blazhko stars are almost as enigmatic now (see Kovács 2009) as when they were discovered over $100 \mathrm{yr}$ ago (Blazhko 1907). The problem in recent years has taken on new significance with the discovery that almost half of all RRab stars are Blazhko variables (Jurcsik et al. 2009c), and that possibly all RRc stars are multiperiodic (Moskalik et al. 2012). These discoveries raise the question whether all RR Lyrae stars might be amplitude and frequency modulators.

The measured amplitude and frequency modulations for the Blazhko RRab stars in our sample are summarized in Table 2, where the stars have been ordered from largest to smallest amplitude modulations, $\Delta A_{1}$ (i.e., from the most extreme Blazhko variability to the least variability). All the quantities are newly derived from both the LC and SC Q0-Q11 photometry, and the uncertainties are at the level of the precisions given. Columns 2-6 contain, respectively, the measured ranges (minimum to maximum values) for: the total amplitude in the $K p$-passband, $\Delta A_{\text {tot }}$; the pulsation period, $\Delta P_{\text {puls }}$; the Fourier amplitude coefficient, $\Delta A_{1}$; the first Fourier phase coefficient, $\Delta \phi_{1}$; and the Fourier phase-difference parameter $\Delta \phi_{31}^{s}$, where $\phi_{31}^{s}=\phi_{3}-3 \phi_{1}$ (adjusted to the value closest to the mean value of 5.8) and the superscript " $s$ " indicates that a sine (rather than a cosine) series was used for the Fourier decomposition (see Equation (1)).

The amplitude modulations, $\Delta A_{\text {tot }}$, range from as large as $\sim 0.82 \mathrm{mag}$ (V445 Lyr, V2178 Cyg) to as small as $\sim 0.02 \mathrm{mag}$ (V838 Cyg, KIC 11125706), corresponding to $2 \%-80 \%$ of $A_{\text {tot }}(\max )$. The stars with the largest amplitude variations tend also to exhibit the largest period variations. The average period range $\Delta P_{\text {puls }}$ is 0.0010 days. Plots of $\Delta A_{\text {tot }}$ versus period, and $\Delta \phi_{31}^{s}$ versus period, show an approximately normal distribution with the largest modulations occurring for those stars with pulsation periods around 0.53 days. In the period $-\Delta \phi_{31}^{s}$ diagram the extreme Blazhko stars V445 Lyr and V2178 Cyg (see Figure 3) are clearly outliers with $\Delta \phi_{31}^{s}$ values considerably larger than for all the other Blazhko stars. Estimates of $\Delta P_{\text {puls }}$ and $\Delta A_{1}$ of RR Lyrae by K11, and V445 Lyr by G12, agree well with the values reported in Table 2 .

\subsubsection{V838 Cyg and KIC 11125706}

When the amplitude modulations of Blazhko stars are very small careful analysis is required to ensure that the fluctuations are not artificial, perhaps caused by the data reduction methods. Such borderline Blazhko stars among the Kepler-field RR Lyr stars include KIC 11125706, V838 Cyg and V349 Lyr (discussed by N11). Previously, KIC 11125706 was found by B10 (page 1588) to have "the lowest amplitude modulation ever detected in an RR Lyrae star," even smaller than the small amplitude modulations seen in RR Gem (Jurcsik et al. 2005) and in SS Cnc (Jurcsik et al. 2006); and V838 Cyg was classified as non-Blazhko by both B10 and N11. We confirm the low amplitude modulations of KIC 11125706, but also find that V838 Cyg exhibits amplitude and frequency modulations and that these are even smaller than those of KIC $11125706\left(\Delta A_{1}=\right.$ $0.0016 \mathrm{mag}$ versus $0.008 \mathrm{mag}$, and $\Delta P_{\text {puls }}=0.0002$ days versus 0.0004 days). The two stars are considerably different in that V838 Cyg has a much higher amplitude and shorter period than KIC 11125706 ( $A_{\text {tot }}=1.10$ mag versus $0.47 \mathrm{mag}$; $P_{\text {puls }}=0.48$ days versus 0.61 days; see Figure 4 below $)$.

Figure 2 shows for V838 Cyg (left) and KIC 11125706 (right) four Fourier variables plotted as time series. The plotted variables are (from bottom to top): the amplitude $A_{1}$ and the phase $\phi_{1}$, both from the first term in the Fourier sine series; the ratio $R_{21}$ of the second and first Fourier amplitudes; and the Fourier phase difference parameter, $\phi_{31}^{s}$. The four variables were obtained by dividing the LC data from Q1 to Q11 into time segments, each segment corresponding to three pulsation 

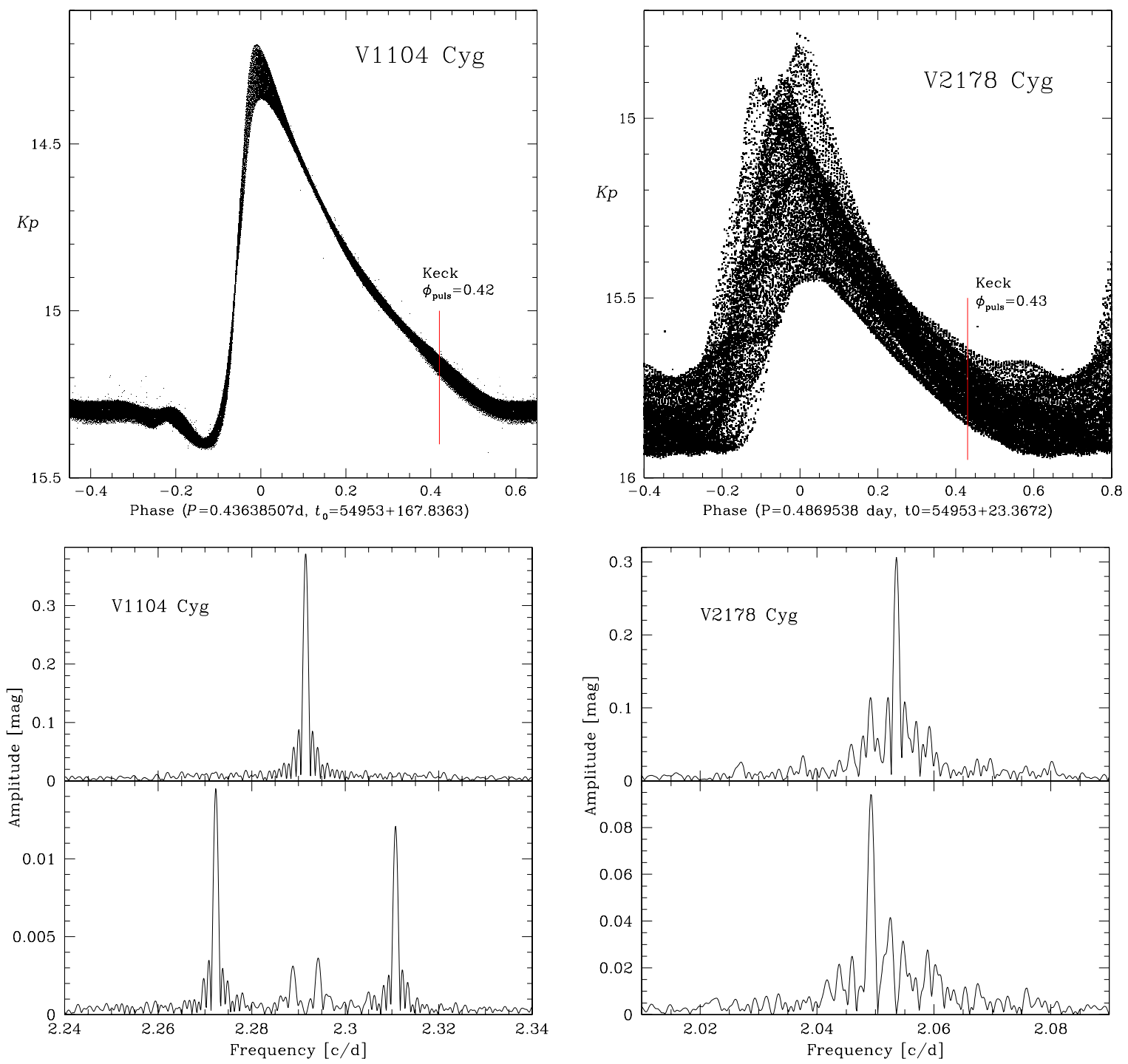

V1104 Cyg (LC:Q1-11)
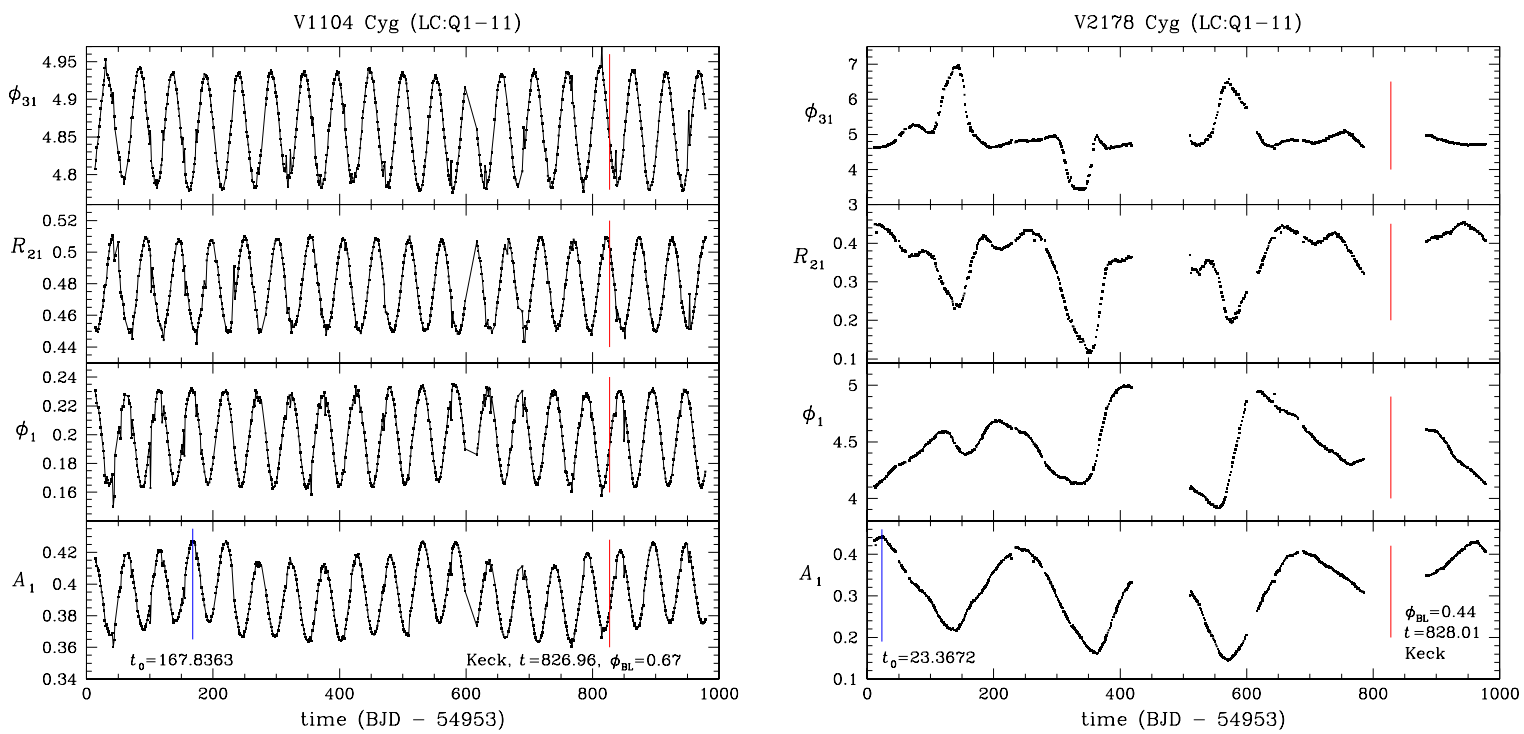

Figure 3. Top: phased light curves for V1104 Cyg (KIC 12155928, left) and V2178 Cyg (KIC 3864443, right) — the mean pulsation phases of the Keck spectroscopic observations are indicated by a vertical line and labeled. Middle: power spectra, where the upper panel shows the Fourier transform in the vicinity of the primary frequency, and the lower panel shows the Fourier transform after prewhitening with the primary frequency and its harmonics. V1104 Cyg is characterized by a symmetric triplet (RR0-BL2) and V2178 Cyg by an possible asymmetric doublet (RR0-BL1?). Bottom: time-series graphs for four Fourier parameters-the vertical lines indicate the assumed times of maximum amplitude and light, $t_{0}$, and the mean times, $t$, and Blazhko phases, $\phi_{\mathrm{BL}}$, at which the Keck observations were made.

(A color version of this figure is available in the online journal.) 

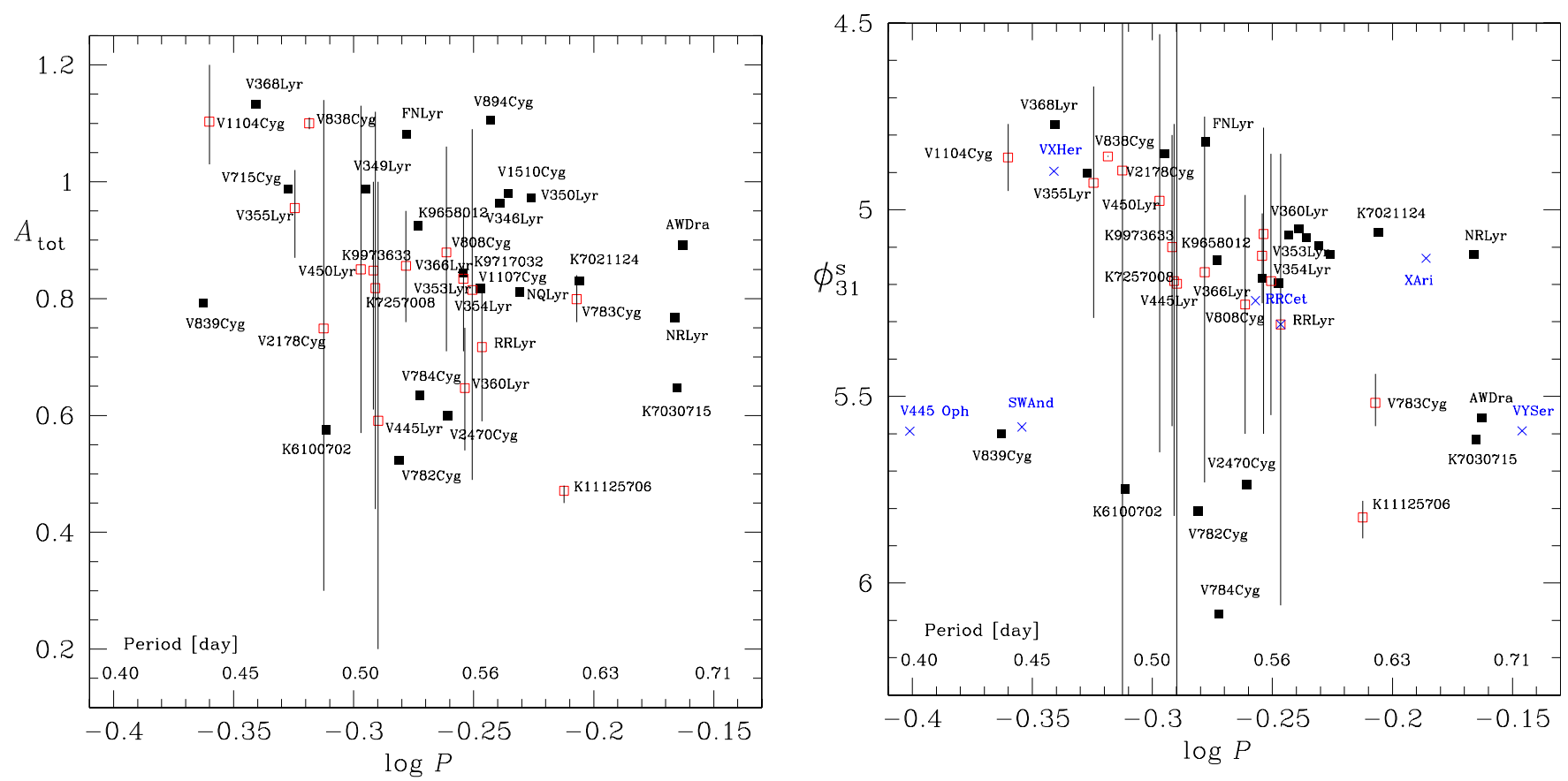

Figure 4. Period-amplitude diagram (left) and period- $\phi_{31}^{s}$ diagram (right) for the non-Blazhko (black squares) and Blazhko (red open squares) RRab stars in the Kepler field. Also represented in the right panel are several Keck spectroscopic standard stars (blue crosses). The periods are pulsation periods, the $A_{\text {tot }}$ are on the $K p$-system, and the Fourier phase parameters $\phi_{31}^{s}$ are from sine-series Fourier decomposition of the Kepler photometry. The $\phi_{31}^{s}$ axis has been reversed to emphasize the similarity of the two diagrams. For the Blazhko stars the vertical lines indicate the measured ranges in their $A_{\text {tot }}$ and $\phi_{31}^{s}$ values (see Table 2), and the (red open) boxes are plotted at the mean $\phi_{31}^{s}$ values.

(A color version of this figure is available in the online journal.)

periods (cf. Figure 5 of N11 where the Kepler stars NQ Lyr and V783 Cyg are plotted). Approximately 40,000 data points were analyzed for each star, and 15 terms were used for the Fourier decompositions.

For both stars the mid-times and Blazhko phases of the respective CFHT spectra are indicated (labeled red vertical lines), as are the adopted $t_{0}$ zero points (labeled blue vertical lines). For KIC 11125706 the large gaps at $t=253-323$ and at $t=600-688$ are because no observations were made in the second two months of Q4 and in Q8. For the same star the blue open squares result from analysis of the 126,955 SC photometric measurements made in Q7-agreement of the $\mathrm{SC}$ and $\mathrm{LC}$ results is excellent. ${ }^{12}$

The modulation periods are obvious and seen to be close to 55 days for V838 Cyg, and 40 days for KIC 11125706 - these are the basic Blazhko periods. In addition to the amplitude modulations seen in the $A_{1}$ and $R_{21}$ panels, the variation of $\phi_{1}$ is indicative of frequency modulation. That the time-series plots for $\phi_{1}$ and $\phi_{31}^{s}$ (which measures the pulse shape) are mirror images of each other is not uncommon (but not always the case, as is seen for V2178 Cyg in Figure 3 below).

On relatively short time scales, such as the $\sim 90$ day baseline of the available SC:Q10 data for V838 Cyg (not plotted), the modulations look sinusoidal; however, closer inspection shows that they are not. That the $A_{1}$ waves are compressed around time $t=200$ and 550 but are wider around $t=400$ and 750 proves that the modulation period for V838 Cyg is varying. Thus the amplitude and frequency modulations are complex. A "Period04" frequency analysis of the LC data identified multiple Blazhko periods of 54, 64, and 47 days, the first being the

\footnotetext{
12 Such agreement is not always the case owing to different normalizations used during the detrending procedure, but the differences appear always to be small (less than $1 \%$ for each variable) and do not affect our main results.
}

most significant. Finally, the cycle-to-cycle amplitude variations seen in the bottom right panel suggest that additional amplitude modulations may be present for KIC 11125705.

Many other Kepler-field Blazhko stars in addition to V838 Cyg and KIC 11125706 have been found to exhibit interesting and complex modulation behaviors. In the next subsection two quite different Blazhko variables (V1104 Cyg and V2178 Cyg) are used to illustrate the time- and frequency-domain methods that were used for deriving the Blazhko periods and Blazhko types.

\subsubsection{Blazhko Periods and Types from Power Spectra}

Blazhko periods can also be inferred from power spectra. In fact, the Blazhko classification scheme of Alcock et al. (2000, 2003) is based solely on characteristic power-spectra patterns. When the Fourier transform of prewhitened photometry shows a single major feature at $f_{1}$, offset from the fundamental pulsation frequency $f_{0}$, the Blazhko type is said to be "RR0-BL1." In this case the Blazhko frequency is $f_{\mathrm{BL}}=\left|f_{1}-f_{0}\right|$, and $P_{\mathrm{BL}}=$ $1 / f_{\mathrm{BL}}$. When the Fourier transform of the prewhitened data shows two major features, symmetric about the fundamental frequency, the type is said to be "RR0-BL2." For such stars $P_{\mathrm{BL}}$ is equal to the reciprocal of the mean frequency shift (assuming that the two features are equidistant from $f_{0}$ ). For other types found in the MACHO surveys see Table 1 of Alcock et al. (2003).

Figure 3 illustrates the time- and frequency-domain methods used here. V1104 Cyg (left panels) is a typical Blazhko star having relatively low amplitude modulation (although much larger than that of KIC 11125706 and V838 Cyg) and a $P_{\mathrm{BL}} \sim 53$ days ( 18 cycles in $\sim 950$ days); and V2178 Cyg (right panels) exhibits extreme amplitude modulation (similar to but not as extreme as V445 Lyr) with $P_{\mathrm{BL}} \sim 235$ days (four cycles in $\sim 940$ days). The top panel for each cluster shows the folded light curves phased with the mean pulsation period (Table 1), 
where amplitude variations and phase shifting (indicative of the frequency modulation) are clearly seen. The middle panels show portions of the power spectra for frequencies near the primary pulsational frequency before (upper graph) and after prewhitening with the primary frequency and its harmonics (lower graph). And the bottom multipanels are time series plots similar to those shown in Figure 2.

The V1104 Cyg light curve (top left panel of Figure 3) is derived from the Q9 SC photometry (138,540 data points), and the power spectra and time series plots are based on the Q1-Q11 LC photometry (43,341 points). A nonlinear least squares fit to the LC data gave an estimated primary frequency of $f_{0}=2.29155411( \pm 5)$ cycles day $^{-1}$ (radial fundamental mode), corresponding to pulsation period $P=0.43638507( \pm 5)$ days. The secondary frequencies are symmetric about $f_{0}$ and occur at $f_{0}-f_{\mathrm{BL}}=2.2723$ cycles day ${ }^{-1}$ and $f_{0}+f_{\mathrm{BL}}=2.3108$ cycles day ${ }^{-1}$, from which we derive the Blazhko frequency, $f_{\mathrm{BL}}=0.01925$ cycles day $^{-1}$ and $P_{\mathrm{BL}}=51.995 \pm 0.005$ days, and classify the star as type RR0-BL2. In the time series plots the variations of all four Fourier parameters are seen to be nearly sinusoidal, with an estimated mean Blazhko period of 52.003 days. The agreement with the $P_{\mathrm{BL}}$ derived from the Fourier transform is excellent, and we adopt the mean of the two values, $51.999 \pm 0.005$ days. The flatness of the $\phi_{1}$ graph lends supports to the derived $P_{\text {puls }}$. An upward sloping line would have resulted if the assumed period had been longer; in this sense the $\phi_{1}$ panels are analogous to $O-C$ diagrams. Finally, the $A_{1}$ panel shows amplitude maxima around $t=200,550$, and 900 days, corresponding to an additional period around $1 \mathrm{yr}$; it is quite possible that this is an artifact of the pre-processing procedure.

The V2178 Cyg light curve (top right panel of Figure 3) is derived from the LC photometry from Q1-Q11 (34,994 data points). The primary frequency, $f_{0}=2.053586 \pm 0.000004 \mathrm{cy}-$ cles day $^{-1}$, corresponds to $P_{\text {puls }}=0.486954 \pm 0.000004$ days. After prewhitening with this frequency and its harmonics, a single strong sidepeak is seen (mag 0.09), accompanied by several weaker sidepeaks (mag $\sim 0.02-0.04$ ). Thus we have what appears to be an asymmetric doublet pattern, and a classification of RR0-BL1. However, this star and to a lesser extent V354 Lyr, which has an uncertain Blazhko type owing to its long $P_{\mathrm{BL}}$, are the only such possible RR0-BL1 types in our sample and it is possible that both are BL2 stars with very asymmetric sidelobes. Such borderline classifications are quite common: Alcock et al. (2003) found that "160 out of the 400 BL1 stars (in the MACHO LMC sample) could be classified as BL2." In any case, the Blazhko period that follows from the frequency difference, $f_{0}-f_{\mathrm{BL}}=0.00433$ cycles day $^{-1}$, is $P_{\mathrm{BL}}=234 \pm 10$ days. The time-series plots are unusual and in general the variations are seen to be considerably more complex than for V1104 Cyg (the gaps in the time-series plots near $t \sim 450$ and 800 are due to no observations having been made in Q6 and in Q10). The alternating up-down bumps seen in the $\phi_{31}^{s}$ panel is, to our knowledge, unique among Blazhko stars.

Diagrams similar to those plotted in Figures 2 and 3 were constructed for all the program stars. While each of the stars seems to be of considerable interest in its own right, many of the findings are only indirectly pertinent to the subject of the metal abundances. Where the diagrams are most relevant is in the adopted mean $\phi_{31}^{s}$ values that go into the photometric $[\mathrm{Fe} / \mathrm{H}]$ analysis (see Section 5), in the pulsation phases that are related to the effective temperatures (and are used to calculate $\gamma$-velocities), and in the Blazhko phases that affect luminosities and effective temperatures needed for deriving spectroscopic
Table 3

Blazhko Periods, Phases and Types

\begin{tabular}{lcccc}
\hline \hline Star & $\begin{array}{c}P_{\mathrm{BL}} \\
(\text { days })\end{array}$ & $t_{0}(\mathrm{BL})$ & $\phi_{\mathrm{BL}}$ & Type \\
$(1)$ & $(2)$ & $(3)$ & $(4)$ & $(5)$ \\
\hline V2178 Cyg & $234 \pm 10$ & 23.3672 & 0.44 & BL1? \\
V808 Cyg & $92.14 \pm 0.01$ & 17.2834 & 0.82 & BL2 \\
V783 Cyg & $27.667 \pm 0.001$ & 22.5439 & 0.93 & BL2 \\
V354 Lyr & $723 \pm 12$ & 292.1590 & 0.74 & unc. \\
V445 Lyr & $54 \pm 1$ & 207.5957 & 0.32 & BL2 \\
RR Lyrae & $39.20 \pm 0.10$ & 325.2263 & 0.77 & BL2 \\
KIC 7257008 & $39.56 \pm 0.08$ & 805.5859 & $\ldots$ & BL2 \\
V355 Lyr & $31.05 \pm 0.04$ & 171.7072 & 0.64 & BL2 \\
V450 Lyr & $123.7 \pm 1.6$ & 43.3226 & 0.34 & BL2 \\
V353 Lyr & {$[71.6,132.6]$} & 129.6820 & 0.74 & BL2x2 \\
V366 Lyr & $62.84 \pm 0.03$ & 373.1915 & 0.24 & BL2 \\
V360 Lyr & $52.07 \pm 0.02$ & 35.9332 & 0.17 & BL2 \\
KIC 9973633 & $73.0 \pm 0.6$ & 827.3655 & 0.99 & BL2 \\
V838 Cyg & {$[54,64,47]$} & 854.9302 & 0.82 & BL2x3 \\
KIC 11125706 & $40.23 \pm 0.03$ & 28.0658 & 0.76 & BL2 \\
V1104 Cyg & $51.999 \pm 0.005$ & 167.8363 & 0.67 & BL2 \\
\hline
\end{tabular}

Notes. The columns contain: (1) star name; (2) Blazhko period (=period of amplitude modulation); (3) time of maximum amplitude (=BJD-2,454,953); (4) Blazhko phase at the mid-time of our spectroscopic observations; (5) RR0 Blazhko type, as defined by Alcock et al. (2000, 2003); the type for V354 Lyr is uncertain owing to its long $P_{\mathrm{BL}}$.

$[\mathrm{Fe} / \mathrm{H}]$ values. Detailed discussions of our findings concerning modulation characteristics of the individual Blazhko stars will be presented elsewhere.

Table 3 gives the estimated Blazhko periods and types of the 16 Kepler-field Blazhko stars. The $P_{\mathrm{BL}}$ estimates were derived by fitting sine functions to time series plots of the amplitudes (see bottom panels of Figures 2 and 3), and from power spectra of the raw and prewhitened photometry, with both methods producing nearly identical estimates. The final $P_{\mathrm{BL}}$ values range from $27.667 \pm 0.001$ days (V783 Cyg) to $723 \pm 12$ days (V354 Lyr), with an average $\left\langle P_{\mathrm{BL}}\right\rangle=111$ days. It is somewhat surprising that none of the stars have Blazhko periods shorter than 27 days, the median $P_{\mathrm{BL}}$ for the 14 Blazhko stars discovered in the Konkoly Blazhko Survey (see Table 2 of Jurcsik et al. 2009c). None of the stars has $P_{\mathrm{BL}}$ as long as the four OGLE-III Galactic Bulge stars, which have $P_{\mathrm{BL}}$ up to 23000 days (see Figure 5 of Soszynski et al. 2011 for light curves). V353 Lyr seems to exhibit doubly periodic modulation with periods of $71.64 \pm 0.06$ days and $132.6 \pm 0.7$ days.

For those stars with $P_{\mathrm{BL}}<100$ days and in common with B10 our estimates of $P_{\mathrm{BL}}$ agree well with the values given in their Table 2. The estimated uncertainties from our analyses are considerably smaller (by factors $\sim 10-100$ times) owing to the much longer time baseline and the inclusion of the SC photometry. The longer baseline also allowed us to derive Blazhko periods for those stars with amplitude modulations that are occurring on time scales longer than 100 days. For example, we derived the following estimates of $P_{\mathrm{BL}}$ for V2178 Cyg, V808 Cyg, V354 Lyr, V450 Lyr: $234 \pm 10,92.14 \pm 0.01,723$ \pm 12 and $123.7 \pm 1.6$ days, respectively. Table 3 also includes the first estimates of $P_{\mathrm{BL}}$ for three stars not in the B10 list: KIC 7257008, KIC 9973633, and V838 Cyg.

\subsubsection{Blazhko Phases}

The Blazhko phase at which the spectra were taken, $\phi_{\mathrm{BL}}$, and the adopted time of zero phase for the Blazhko cycles, $t_{0}(\mathrm{BL})$, 
assumed to be when the pulsation amplitude was largest, are also given in Table 3 . The $t_{0}(\mathrm{BL})$ are identical to the values given in Table 1 , but are more conveniently expressed to correspond with the time-series graphs (see Figures 2 and 3). For a given star the Blazhko phases were necessarily random.

If the $P_{\mathrm{BL}}$ and $A_{\mathrm{tot}}$ were time invariant then any time of maximum amplitude could have been chosen as the zero-point for the Blazhko variations. However, this is not the case (see Table 2) since many of the Blazhko stars also have multiple Blazhko frequencies and complex power spectra. For such stars the $\phi_{\mathrm{BL}}$ depend on many factors, and in these cases local maxima before and after the times of the observed spectra were identified and these were used to compute the Blazhko phase.

The time series plots shown in Figures 2 and 3 serve to illustrate the $\phi_{\mathrm{BL}}$ determinations. Four spectra of V838 Cyg were taken at CFHT, between BJD-54,953 = 460.79 and 460.82 , with a mid-time of 460.80 . The times of maximum amplitude immediately preceding and following 461 are at 415 and 472, respectively. Thus $\phi_{\mathrm{BL}}=0.82$ for V838 Cyg. V1104 Cyg and V2178 Cyg were both observed at Keck, with mid-times of the observations at 826.96 and 828.01, respectively. Since the times of maximum amplitude immediately before and after 826.96 days occur at 791.1 and 844.8 the $\phi_{\mathrm{BL}}$ for V1104 Cyg is 0.67. A similar calculation made for V2178 Cyg gave $\phi_{\mathrm{BL}}=0.44$.

\subsection{Period-Amplitude and Period- $\phi_{31}$ Diagrams}

The early studies by Oosterhoff (1939, 1944), Arp (1955), and Preston (1959) established that in the period-amplitude diagram for RR Lyrae stars there is a separation by metal abundance, with metal-poor RRab stars tending to have longer pulsation periods at a given amplitude than metal-rich RRab stars. This effect has been used to better understand the Oosterhoff dichotomy and to derive metallicities for RR Lyrae stars found in different environments, such as in globular clusters (Sandage 1981, 1990, 2004; Cacciari et al. 2005), in different fields of our Galaxy, and in other galaxies. The effect is now known to be due to the lower metal abundance stars having greater luminosities and therefore longer periods (see Sandage 2010; Bono et al. 2007), a result which is consistent with the recent Warsaw convective pulsation hydro-models (see Figure 14 of N11).

In the 1980s Simon and his collaborators introduced Fourier decomposition techniques for describing the shapes of RR Lyr light curves, and established useful correlations between various Fourier parameters and [Fe/H], mass, luminosity, and other physical parameters (Simon \& Lee 1981; Simon \& Teays 1982; Simon 1985, 1988; Simon \& Clement 1993). Since the mid1990s Kovács and Jurcsik and their collaborators (Kovács \& Zsoldos 1995; Jurcsik \& Kovács 1996, hereafter JK96; Kovács \& Jurcsik 1996; Kovács \& Walker 2001; Kovács 2005) have expanded upon these Fourier ideas and have produced useful empirical equations that describe the relationships between RRab light curve parameters and physical characteristics. In particular, JK96 derived a $P-\phi_{31}^{s}-[\mathrm{Fe} / \mathrm{H}]$ relation (their Equation (3)), which is often used to estimate the metallicities of RRab stars. More recently, Morgan et al. (2007, hereafter M07) derived analogous equations for RRc stars.

In Figure 4 the $\log P-A_{\text {tot }}$ and $\log P-\phi_{31}^{s}$ diagrams are plotted for the Kepler-field RRab stars. The pulsation periods, $A_{\text {tot }}(K p)$ and $\phi_{31}^{s}(K p)$ are the new values given in Table 1 , and the diagrams include both non-Blazhko (black squares) and Blazhko stars (red open squares). By reversing the direction of the $\phi_{31}^{s}$ axis one observes directly the similarity of the two diagrams (see
Sandage 2004) and that those stars with the largest amplitudes tend to have the most asymmetric light curves, i.e., the smallest $\phi_{31}^{s}$ values. In both diagrams, all but a few of the most crowded points have been labeled with the star names. For the two most extreme Blazhko stars, V445 Lyr and V2178 Cyg, the $\phi_{31}^{s}$ ranges exceed the range of the plotted $y$-axis. The RRc stars in the Kepler field are off-scale to the left of both graphs, with small amplitudes, large $\phi_{31}^{s}$ values, and periods shorter than 0.4 days.

Several trends are apparent in Figure 4, the most obvious being the similarity of the two diagrams. There is also an apparent separation of the stars into two groups, discriminated better by $P_{\text {puls }}$ and $\phi_{31}^{s}$ than by $P_{\text {puls }}$ and $A_{\text {tot }}$. Most of the stars are observed to have $A_{\text {tot }}>0.8$ mag and $\phi_{31}^{s}<5.4 \mathrm{rad}$. According to the metallicity correlations mentioned above these are metal-poor stars with $[\mathrm{Fe} / \mathrm{H}]<-1.0$ dex. The other group, those shorter-period stars with lower amplitudes and higher $\phi_{31}^{s}$ values, are expected to be more metal-rich, with $[\mathrm{Fe} / \mathrm{H}]>-1.0$ dex. For a fixed metallicity as the pulsation period increases the stars tend to have smaller amplitudes and more sinusoidal light curves (i.e., smaller $\phi_{31}^{s}$ values).

When all the stars in a given sample are suspected of having the same or similar metallicities (e.g., in globular clusters with narrow RGBs), or when there exist independent spectroscopic metal abundances for the sample stars, then diagonal lines can be fit to the observational data (e.g., Sandage 2004; Cacciari et al. 2005; N11). Such diagonal trends are evident in the Figure 4 $\log P-\phi_{31}^{s}$ diagram, especially when the spectroscopic standard stars (blue crosses) are included in the diagram. This diagram also suggests that the new non-Blazhko star V839 Cyg, and the low-amplitude-modulation star KIC 11125706 (Figure 2) are metal rich. Indeed, we show below that V839 Cyg is metal-rich, with $[\mathrm{Fe} / \mathrm{H}]=-0.05 \pm 0.14 \mathrm{dex}$; however, the CFHT spectra of KIC 11125706, with $[\mathrm{Fe} / \mathrm{H}]=-1.09 \pm 0.08$ dex (see Table 7), indicate that it probably belongs to the metal-poor group.

The Blazhko stars require special consideration. They are represented in Figure 4 by their mean pulsation periods and their mean $A_{\text {tot }}$ or $\phi_{31}^{s}$ values (red open squares), with vertical lines spanning the measured ranges of $A_{\text {tot }}$ and $\phi_{31}^{s}$. If metal abundances are derived by substituting the mean $\phi_{31}^{s}$ values given in Table 1 into the JK96 $P-\phi_{31}^{s}-[\mathrm{Fe} / \mathrm{H}]$ relation, then the resulting photometric $[\mathrm{Fe} / \mathrm{H}]$ values suggest that almost all of the Blazhko variables are metal-poor; indeed, this is supported by the spectroscopic metallicities (next section), and runs contrary to the suggestion by Moskalik \& Poretti (2003) that the incidence of Blazhko variables increases with $[\mathrm{Fe} / \mathrm{H}]$. Also, since the Blazhko stars appear to have, at a given period, lower mean $A_{\text {tot }}$ values and higher mean $\phi_{31}^{s}$ values than the non-Blazhko stars, it appears that the Kepler Blazhko stars must be more metal-rich, on average, than the non-Blazhko stars. The validity of these conclusions depends on the applicability of the JK96 formula, and on the appropriateness of the assumed $\phi_{31}^{s}$ values, the latter being particularly true for the most extreme Blazhko stars (V445 Lyr, V2178 Cyg) where the $\phi_{31}^{s}$ show large modulations. These topics will be discussed further in Section 5 .

\section{CFHT AND KECK SPECTROSCOPY}

High-resolution spectroscopic observations were made of the Kepler-field RR Lyrae stars using the CFHT 3.6 m telescope and the Keck-I 10 m telescope (W.M. Keck Observatory), both located on Mauna Kea, Hawaii. A total of 123 spectra were 
taken of 42 stars and in most cases the derived spectroscopic metal abundances are the first available estimates.

Since a primary goal of this study was derivation of $[\mathrm{Fe} / \mathrm{H}]_{\text {spec }}$ values, spectra were taken at pulsation phases between $\sim 0.2$ and $\sim 0.5$, at the changeover from outflow to infall. Away from these phases the spectra are increasingly affected by velocitygradients in the atmosphere and shock waves that broaden the spectral lines (see Preston 2009, 2011; K11; For et al. 2011). The pulsation phases for the mid-times of the individual spectra are given in Tables 4 and 5 below and were derived using the updated pulsation periods and $t_{0}$ values that were calculated using Q0-Q11 photometry. Nearly $80 \%$ of the spectra were acquired at phases between 0.25 and 0.55 (see upper panel of Figure 5). The overall median and mean $\phi_{\text {puls }}$ are 0.35 and 0.38 , respectively, with $\sigma=0.13$; the mean $\phi_{\text {puls }}$ for the 54 Keck spectra is larger than that for the 68 CFHT spectra ( 0.48 versus $0.30)$.

\subsection{CFHT 3.6 m ESPaDOnS Spectra}

The CFHT spectra were acquired from 2010 July-December using the ESPaDOnS prism cross-dispersed échelle spectrograph in the "star+sky" non-polarimetric mode. Two optical fibers fed the star and sky images from the Cassegrain focus to the Coudé focus, then onto a Bowen-Walraven image slicer at the entrance of the spectrograph. The resolving power was $R=\lambda / \Delta \lambda \sim 65,000$, which corresponds to a spectral resolution of $\Delta \lambda \sim 0.008 \mathrm{~nm}$ at $\lambda=517 \mathrm{~nm}$ (Mg triplet). The spectra are spread over 40 grating orders (on an EEV1 CCD chip), with wavelength coverage from 370 to $1048 \mathrm{~nm}$, an average reciprocal dispersion of $0.00318 \mathrm{~nm}_{\text {pixel }}^{-1}$, and $\sim 3$ pixels per resolution element.

Eighteen of the brightest RR Lyrae stars in the Kepler field were observed at CFHT (Service Observing). The total observing time amounted to $20 \mathrm{hr}$. The observations were made under photometric skies for all but three of the stars (V355 Lyr, V838 Cyg, KIC 7030715), and at various hour angles and airmasses. The seeing was rarely better than 1 arcsec. Multiple spectra were taken for most stars, usually with exposure times of $4 \times 900 \mathrm{~s}$; for the faintest observed stars $(14.5<K p<15.0)$ the exposure times were increased to $4 \times 1200 \mathrm{~s}$.

The "Upena" software, which uses "Libre-ESpRIT,"13 was used to pre-process the CFHT spectra-i.e., perform bias subtraction and flat fielding, subtract the sky and scattered light, identify the echelle orders and perform optimal image extraction, make the wavelength calibrations (using spectra of a Th-Ar lamp), calculate the signal-to-noise ratios ( $\mathrm{S} / \mathrm{Ns}$ per pixel), and make the necessary heliocentric corrections for Earth's motion. The individual spectra were normalized and the overlapping échelle orders merged using the VWA "rainbow" widget (Bruntt et al. 2010a, 2010b). For those stars with multiple observations the normalized spectra were co-added to increase the $\mathrm{S} / \mathrm{Ns}$. Details of the individual spectra are given in Table 4 . The $\mathrm{S} / \mathrm{N}$ per pixel values (Column 5) were measured at $570 \mathrm{~nm}^{14}$ and range from 7-96, with typical values $\sim 20-30$. The

\footnotetext{
13 The original "ESpRIT" program is described by Donati et al. (1997); "Libre-ESpRIT" is the current release used at CFHT and documented at http://www.cfht.hawaii.edu/Instruments/Spectroscopy/Espadons/Espadons_ esprit.html.

14 The $\mathrm{S} / \mathrm{N}$ ratio is largest in the interval $550-580 \mathrm{~nm}$, but does not vary by more than $\sim 20 \%$ over the interval $500-900 \mathrm{~nm}$. Since one resolution element is approximately 1.4 pixels, or $2.6 \mathrm{~km} \mathrm{~s}^{-1}$, the $\mathrm{S} / \mathrm{N}$ per resolution element are $\sim 1.2$ times larger.
}
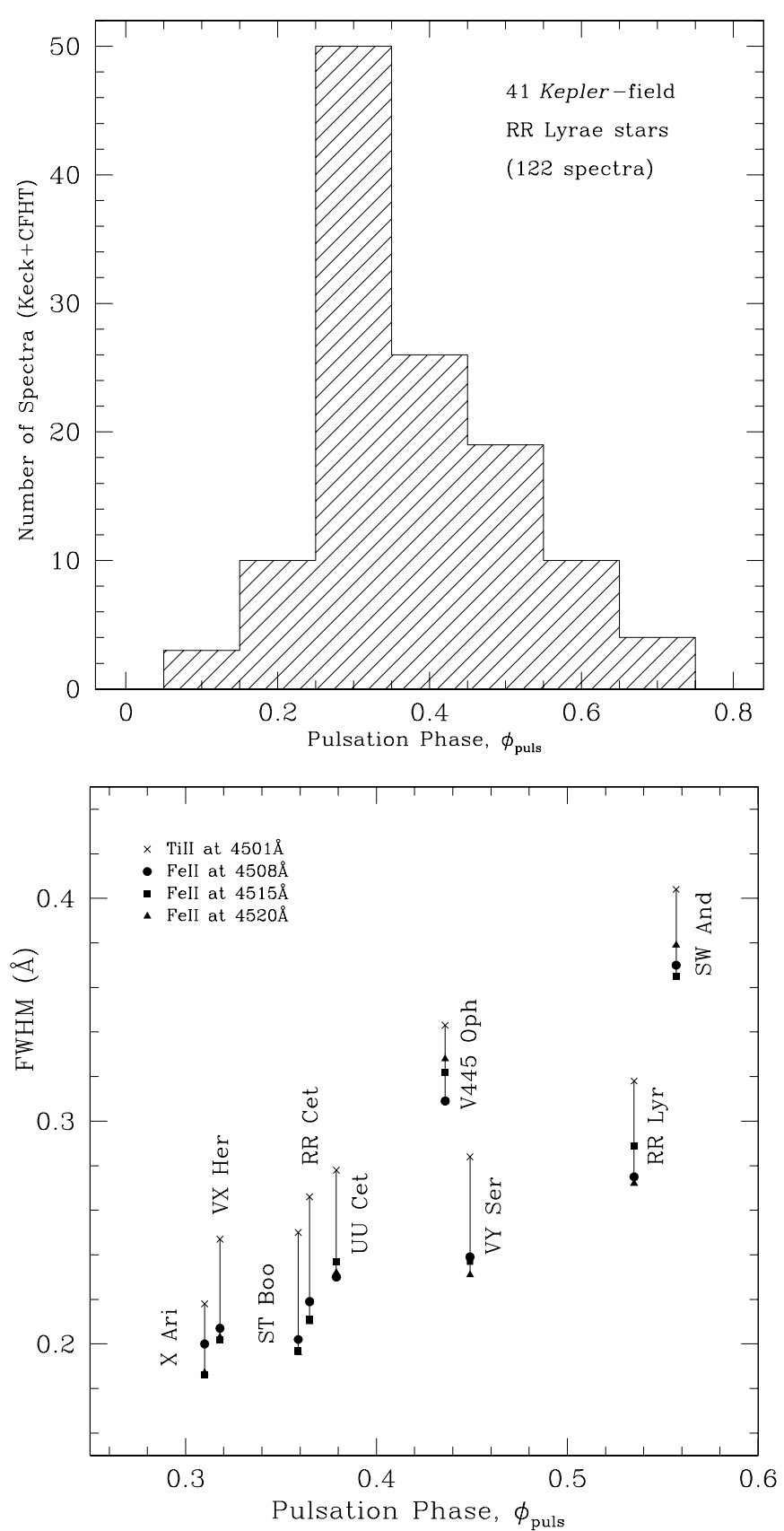

Figure 5. Top: histogram of the observed pulsation phases for the 41 Keplerfield RR Lyrae stars, based on the 122 spectra taken with the CFHT $3.6 \mathrm{~m}$ and Keck-I 10 m telescopes. Bottom: FWHM values for four spectral lines near $450 \mathrm{~nm}$, as a function of the pulsation phase. The points plotted are for the bright RR Lyrae standard stars observed with Keck, and the FWHM values were measured using the IRAF "splot" routine. The narrowest lines are seen to occur at phases between 0.3 and 0.4 , and for a given phase the three Fe II lines (at $450.8,451.5$ and $452.0 \mathrm{~nm}$ ) are narrower than the Ti II line (at $450.1 \mathrm{~nm}$ ).

FWHM values (Column 6) were measured using the Fe II lines at $450.8,451.5$, and $452.0 \mathrm{~nm}$, and range from $\sim 100-400 \mathrm{m \AA} .^{15}$

Co-added spectra for the 16 CFHT stars for which metallicities could be derived are plotted in Figure 6, where the wavelength range $515<\lambda<520 \mathrm{~nm}$ contains the green $\mathrm{Mg}$ I triplet lines. The spectra have been corrected for Doppler shifts, and,

\footnotetext{
15 The measured FWHM values are related to the true (or instrumentcorrected) values according to $\mathrm{FWHM}_{\text {true }}=\left(\mathrm{FWHM}_{\text {meas }}^{2}-\mathrm{FWHM}_{\mathrm{instr}}^{2}\right)^{1 / 2}$,

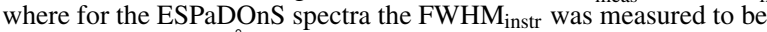
$\sim 2.0$ pixels, or $64 \mathrm{~m} \AA$.
} 
Table 4

CFHT ESPaDOnS Spectra of Kepler RR Lyrae Stars

\begin{tabular}{|c|c|c|c|c|c|c|c|c|c|}
\hline Star & $\begin{array}{l}\text { Spectrum } \\
\text { No. } \\
\text { (2) }\end{array}$ & $\begin{array}{l}t_{\exp } \\
(\mathrm{s}) \\
(3)\end{array}$ & $\begin{array}{c}\text { Observation } \\
\text { Date (UT) } \\
(4)\end{array}$ & $\begin{array}{l}\mathrm{S} / \mathrm{N} \\
(5)\end{array}$ & $\begin{array}{c}\text { FWHM } \\
(\mathrm{m \AA} \AA \\
(6)\end{array}$ & $\begin{array}{l}\text { AM } \\
(7)\end{array}$ & $\begin{array}{c}\text { HJD (Mid) } \\
2,400,000+ \\
\text { (8) }\end{array}$ & $\begin{array}{c}\phi_{\text {puls }} \\
(9)\end{array}$ & $\begin{array}{c}\text { RV (fxcor) } \\
\left(\mathrm{km} \mathrm{s}^{-1}\right) \\
(10)\end{array}$ \\
\hline \multirow[t]{4}{*}{ KIC 6100702} & 1218711 & 900 & 2010 Jul 30 & 23 & $308(10)$ & 1.13 & 55407.9264 & 0.227 & $-54.16(20)$ \\
\hline & 1218712 & 900 & $2010 \mathrm{Jul} 30$ & 22 & $269(08)$ & 1.17 & 55407.9406 & 0.256 & $-52.38(17)$ \\
\hline & 1218713 & 900 & 2010 Jul 30 & 21 & $283(06)$ & 1.20 & 55407.9515 & 0.279 & $-50.54(17)$ \\
\hline & 1218714 & 900 & $2010 \mathrm{Jul} 30$ & 20 & 249 (20) & 1.24 & 55407.9625 & 0.301 & $-47.85(17)$ \\
\hline \multirow[t]{4}{*}{ AW Dra } & 1218715 & 900 & 2010 Jul 30 & 32 & $226(23)$ & 1.34 & 55407.9754 & 0.287 & $-197.85(18)$ \\
\hline & 1218716 & 900 & 2010 Jul 30 & 31 & $210(16)$ & 1.34 & 55407.9863 & 0.302 & $-196.25(21)$ \\
\hline & 1218717 & 900 & 2010 Jul 30 & 32 & $200(03)$ & 1.44 & 55407.9973 & 0.318 & $-194.65(21)$ \\
\hline & 1218718 & 900 & $2010 \mathrm{Jul} 30$ & 31 & $198(08)$ & 1.51 & 55408.0082 & 0.334 & $-193.70(22)$ \\
\hline \multirow[t]{4}{*}{ FN Lyr } & 1218719 & 900 & 2010 Jul 30 & 32 & $226(21)$ & 1.49 & 55408.0204 & 0.265 & $-261.20(23)$ \\
\hline & 1218720 & 900 & $2010 \mathrm{Jul} 30$ & 33 & $203(03)$ & 1.57 & 55408.0313 & 0.286 & $-258.57(21)$ \\
\hline & 1218721 & 900 & $2010 \mathrm{Jul} 30$ & 32 & $236(12)$ & 1.68 & 55408.0423 & 0.307 & $-256.85(23)$ \\
\hline & 1218722 & 900 & 2010 Jul 30 & 29 & $217(18)$ & 1.81 & 55408.0533 & 0.328 & $-254.67(16)$ \\
\hline \multirow[t]{4}{*}{ V894 Cyg } & 1219066 & 900 & 2010 Aug 1 & 27 & $178(18)$ & 1.63 & 55409.7372 & 0.354 & $-234.14(21)$ \\
\hline & 1219067 & 900 & 2010 Aug 1 & 29 & $214(28)$ & 1.54 & 55409.7482 & 0.383 & $-232.47(18)$ \\
\hline & 1219068 & 900 & 2010 Aug 1 & 29 & $189(18)$ & 1.47 & 55409.7591 & 0.402 & $-230.38(18)$ \\
\hline & 1219069 & 900 & 2010 Aug 1 & 29 & $196(11)$ & 1.40 & 55409.7702 & 0.422 & $-228.90(21)$ \\
\hline \multirow[t]{4}{*}{ NQ Lyr } & 1219070 & 900 & 2010 Aug 1 & 27 & $198(09)$ & 1.23 & 55409.7836 & 0.301 & $-72.45(18)$ \\
\hline & 1219071 & 900 & 2010 Aug 1 & 27 & $183(34)$ & 1.19 & 55409.7945 & 0.320 & $-70.47(15)$ \\
\hline & 1219072 & 900 & 2010 Aug 1 & 27 & $185(12)$ & 1.16 & 55409.8054 & 0.339 & $-68.43(22)$ \\
\hline & 1219073 & 900 & 2010 Aug 1 & 26 & $152(12)$ & 1.14 & 55409.8164 & 0.357 & $-66.97(13)$ \\
\hline \multirow[t]{4}{*}{ NR Lyr } & 1219477 & 900 & 2010 Aug 2 & 35 & $222(28)$ & 1.24 & 55410.9703 & 0.274 & $-124.30(26)$ \\
\hline & 1219478 & 900 & 2010 Aug 2 & 35 & 147 (24) & 1.29 & 55410.9813 & 0.290 & $-123.20(19)$ \\
\hline & 1219479 & 900 & 2010 Aug 2 & 36 & $129(05)$ & 1.34 & 55410.9922 & 0.306 & $-121.82(15)$ \\
\hline & 1219480 & 900 & 2010 Aug 2 & 35 & $094(04)$ & 1.41 & 55411.0032 & 0.322 & $-119.42(28)$ \\
\hline \multirow[t]{4}{*}{ V355 Lyr } & 1219876 & 1200 & 2010 Aug 5 & 17 & $309(05)$ & 1.37 & 55413.7353 & 0.144 & $-240.73(16)$ \\
\hline & 1219877 & 1200 & 2010 Aug 5 & 16 & $161(34)$ & 1.29 & 55413.7497 & 0.175 & $-238.03(34)$ \\
\hline & 1219878 & 1200 & 2010 Aug 5 & 15 & $195(50)$ & 1.24 & 55413.7641 & 0.205 & $-233.78(17)$ \\
\hline & 1219879 & 1200 & 2010 Aug 5 & 16 & 219 (13) & 1.19 & 55413.7785 & 0.236 & $-231.82(11)$ \\
\hline \multirow[t]{4}{*}{ V838 Cyg } & 1219880 & 900 & 2010 Aug 5 & 14 & $173(34)$ & 1.23 & 55413.7924 & 0.328 & $-209.05(21)$ \\
\hline & 1219881 & 900 & 2010 Aug 5 & 14 & $231(47)$ & 1.21 & 55413.8033 & 0.351 & $-206.91(25)$ \\
\hline & 1219882 & 900 & 2010 Aug 5 & 13 & $140(60)$ & 1.18 & 55413.8143 & 0.374 & $-205.73(26)$ \\
\hline & 1219883 & 900 & 2010 Aug 5 & 13 & $166(06)$ & 1.17 & 55413.8252 & 0.396 & -202.29 (19) \\
\hline \multirow[t]{4}{*}{ V2470 Cyg } & 1220136 & 900 & 2010 Aug 6 & 23 & $250(13)$ & 1.37 & 55414.7545 & 0.289 & $-57.06(18)$ \\
\hline & 1220137 & 900 & 2010 Aug 6 & 23 & $225(17)$ & 1.32 & 55414.7654 & 0.309 & $-55.16(22)$ \\
\hline & 1220138 & 900 & 2010 Aug 6 & 23 & $255(07)$ & 1.28 & 55414.7764 & 0.329 & $-53.38(22)$ \\
\hline & 1220139 & 900 & 2010 Aug 6 & 23 & $257(16)$ & 1.24 & 55414.7874 & 0.349 & $-51.59(25)$ \\
\hline \multirow[t]{4}{*}{ KIC 11125706} & 1220140 & 900 & 2010 Aug 6 & 57 & $228(04)$ & 1.19 & 55414.8033 & 0.308 & $-69.42(20)$ \\
\hline & 1220141 & 900 & 2010 Aug 6 & 59 & $229(07)$ & 1.17 & 55414.8142 & 0.326 & $-68.08(22)$ \\
\hline & 1220142 & 900 & 2010 Aug 6 & 59 & $223(05)$ & 1.16 & 55414.8251 & 0.343 & $-66.58(22)$ \\
\hline & 1220143 & 900 & 2010 Aug 6 & 61 & $236(03)$ & 1.15 & 55414.8361 & 0.361 & $-65.19(22)$ \\
\hline KIC $3868420^{a}$ & 1220147 & 600 & 2010 Aug 6 & 96 & $359(09)$ & 1.06 & 55414.9016 & 0.173 & $-27.63(16)$ \\
\hline KIC 9453114 & 1220148 & 900 & 2010 Aug 6 & 29 & $283(13)$ & 1.16 & 55414.9120 & 0.241 & $-152.09(20)$ \\
\hline \multirow[t]{3}{*}{$(\mathrm{RRc})$} & 1220149 & 900 & 2010 Aug 6 & 30 & $320(60)$ & 1.19 & 55414.9230 & 0.271 & $-153.53(22)$ \\
\hline & 1220150 & 900 & 2010 Aug 6 & 30 & 375 (42) & 1.22 & 55414.9339 & 0.301 & $-152.31(19)$ \\
\hline & 1220151 & 900 & 2010 Aug 6 & 29 & $267(42)$ & 1.25 & 55414.9449 & 0.331 & $-151.70(15)$ \\
\hline \multirow[t]{4}{*}{ V1104 Cyg } & 1259187 & 1200 & 2010 Nov 16 & 19 & $316(57)$ & 1.33 & 55516.6860 & 0.117 & $-325.49(63)$ \\
\hline & 1259188 & 1200 & 2010 Nov 16 & 20 & 294 (13) & 1.39 & 55516.7004 & 0.150 & $-320.87(69)$ \\
\hline & 1259189 & 1200 & 2010 Nov 16 & 19 & $287(49)$ & 1.46 & 55516.7148 & 0.184 & $-318.40(24)$ \\
\hline & 1259190 & 1200 & 2010 Nov 16 & 15 & $380(10)$ & 1.55 & 55516.7293 & 0.217 & $-313.73(38)$ \\
\hline V1510 Cyg & 1259908 & 1200 & 2010 Nov 18 & 20 & $250(50)$ & 1.19 & 55518.6891 & 0.316 & $-350.35(40)$ \\
\hline & 1259909 & 1200 & 2010 Nov 18 & 20 & $220(40)$ & 1.24 & 55518.7035 & 0.341 & $-348.22(18)$ \\
\hline & 1259910 & 1200 & 2010 Nov 18 & 19 & $250(50)$ & 1.29 & 55518.7179 & 0.366 & $-345.90(53)$ \\
\hline & 1259911 & 1200 & 2010 Nov 18 & 19 & $\ldots$ & 1.36 & 55518.7323 & 0.391 & $-343.55(20)$ \\
\hline V783 Cyg & 1261460 & 1200 & 2010 Nov 25 & 17 & $200(60)$ & 1.24 & 55525.6867 & 0.324 & $-197.26(27)$ \\
\hline & 1261461 & 1200 & 2010 Nov 25 & 16 & $250(100)$ & 1.30 & 55525.7011 & 0.347 & $-195.38(22)$ \\
\hline & 1261462 & 1200 & 2010 Nov 25 & 16 & $250(70)$ & 1.38 & 55525.7156 & 0.370 & $-193.16(22)$ \\
\hline & 1261463 & 1200 & 2010 Nov 25 & 17 & $250(100)$ & 1.48 & 55525.7300 & 0.394 & $-190.49(36)$ \\
\hline KIC 8832417 & 1262172 & 900 & 2010 Nov 27 & 30 & $243(74)$ & 1.59 & 55527.7291 & 0.223 & $-28.52(15)$ \\
\hline$(\mathrm{RRc})$ & 1262173 & 900 & 2010 Nov 27 & 29 & $293(09)$ & 1.69 & 55527.7400 & 0.267 & $-27.07(16)$ \\
\hline & 1262174 & 900 & 2010 Nov 27 & 28 & $324(33)$ & 1.82 & 55527.7509 & 0.311 & $-25.86(17)$ \\
\hline & 1262175 & 900 & 2010 Nov 27 & 27 & $351(28)$ & 1.96 & 55527.7619 & 0.355 & $-25.41(51)$ \\
\hline V808 Cyg & 1265907 & 1200 & 2010 Dec 15 & 9 & $\ldots$ & 1.71 & 55545.6950 & 0.256 & $\ldots$ \\
\hline & 1265908 & 1200 & 2010 Dec 15 & 8 & $\ldots$ & 1.89 & 55545.7094 & 0.283 & $\ldots$ \\
\hline
\end{tabular}


Table 4

(Continued)

\begin{tabular}{|c|c|c|c|c|c|c|c|c|c|}
\hline $\begin{array}{l}\text { Star } \\
\text { (1) }\end{array}$ & $\begin{array}{l}\text { Spectrum } \\
\text { No. } \\
(2)\end{array}$ & $\begin{array}{l}t_{\exp } \\
(\mathrm{s}) \\
(3)\end{array}$ & $\begin{array}{c}\text { Observation } \\
\text { Date (UT) } \\
\text { (4) }\end{array}$ & $\begin{array}{l}S / N \\
(5)\end{array}$ & $\begin{array}{l}\text { FWHM } \\
(\mathrm{m} \AA) \\
(6)\end{array}$ & $\begin{array}{l}\mathrm{AM} \\
(7)\end{array}$ & $\begin{array}{c}\text { HJD (Mid) } \\
2,400,000+ \\
\text { (8) }\end{array}$ & $\begin{array}{c}\phi_{\text {puls }} \\
(9)\end{array}$ & $\begin{array}{c}\mathrm{RV} \text { (fxcor) } \\
\left(\mathrm{km} \mathrm{s}^{-1}\right) \\
(10)\end{array}$ \\
\hline \multirow{6}{*}{ KIC 7030715} & 1265909 & 1200 & $2010 \operatorname{Dec} 15$ & 8 & $\ldots$ & 2.14 & 55545.7238 & 0.309 & $\ldots$ \\
\hline & 1265910 & 1200 & 2010 Dec 15 & 7 & $\ldots$ & 2.47 & 55545.7382 & 0.335 & $\ldots$ \\
\hline & 1266124 & 900 & 2010 Dec 16 & 23 & $330(140)$ & 1.89 & 55546.6890 & 0.238 & $-373(8)$ \\
\hline & 1266125 & 900 & 2010 Dec 16 & 26 & $222(34)$ & 2.06 & 55546.6999 & 0.254 & $-365.41(17)$ \\
\hline & 1266126 & 900 & 2010 Dec 16 & 23 & $208(02)$ & 2.27 & 55546.7109 & 0.270 & $-364.41(48)$ \\
\hline & 1266127 & 900 & 2010 Dec 16 & 14 & $\ldots$ & 2.53 & 55546.7218 & 0.286 & $-361.98(62)$ \\
\hline
\end{tabular}

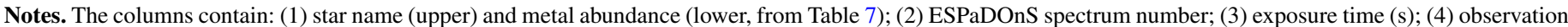

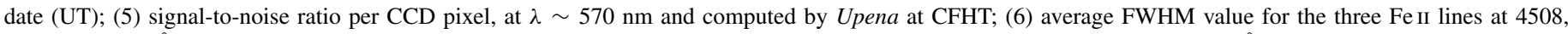

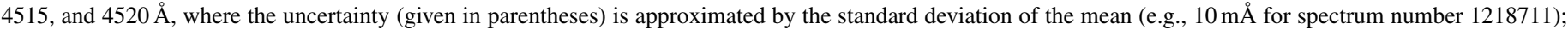

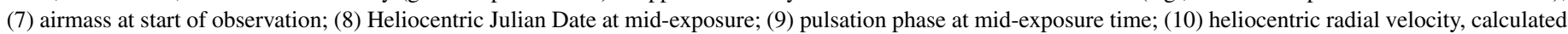
using the IRAF "fxcor" routine.

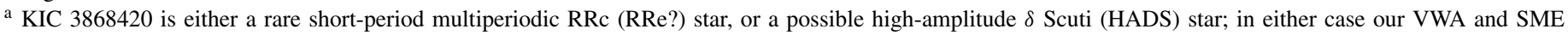
analyses indicate that it is metal rich.

to illustrate the effect of increasing metallicity, are ordered by $[\mathrm{Fe} / \mathrm{H}]$. The spectra of V1104 Cyg $(K p=15.03)$ and V808 Cyg $(K p=15.36)$ were of insufficient quality to permit the derivation of metal abundances (but were sufficient for deriving RVs for V1104 Cyg) and are not shown; both stars were re-observed with the Keck telescope.

\subsection{Keck-I 10 m HIRES Spectra}

The faintest Kepler-field RR Lyrae stars were observed on the nights of 2011 August 4/5, 5/6 and 6/7 with the HIRES echelle spectrograph (Vogt et al. 1994) mounted on the Keck-I $10 \mathrm{~m}$ telescope (see Cohen \& Melendez 2005a, 2005b; Cohen $\&$ Huang 2009)—see Table 5 for details. The spectrograph was used in the HIRES-r configuration and thus optimized for long wavelength observations (QE $>60 \%$ between 400 and $900 \mathrm{~nm}$ ). A slit of width 1.15 arcsec and length 7 arcsec (C5 decker, no filters) was used, resulting in resolving power $R \sim 36,000$ $(\Delta \lambda \sim 0.014 \mathrm{~nm}$ at $517 \mathrm{~nm})$ over the range $389-836 \mathrm{~nm}$. The average reciprocal dispersion is $0.00230 \mathrm{~nm} \mathrm{pixel}{ }^{-1}(140 \mathrm{~nm}$ spread over 60,882 pixels) and the number of pixels per resolution element is $\sim 6$. The spectra were spread over a mosaic of three MIT/Lincoln Labs $2048 \times 4096$ CCD chips. ${ }^{16}$ The $\lambda$ scale was calibrated using ThAr lamp exposures taken at the beginning and end of each night; measurements of the widths of typical emission lines suggest an instrumental FWHM of $5.6 \pm 0.3$ pixels, corresponding to $129 \pm 7 \mathrm{~m} \AA$. To reduce phasesmearing and potential cosmic ray problems exposure times for the program stars were never longer than $1200 \mathrm{~s}$, and usually multiple exposures were taken and co-added. The Mauna Kea Echelle Extraction (MAKEE ${ }^{17}$ ) reduction package was used to pre-process the data.

In addition to the program stars we observed nine bright RR Lyr standard stars studied by Layden (1994, hereafter L94) and Clementini et al. (1995, hereafter C95), and three red

\footnotetext{
16 The blue chip (CCD1) covers the range $389 \mathrm{~nm}$ to $529 \mathrm{~nm}$ (24 spectral orders) and includes $\mathrm{H} \beta, \mathrm{H} \gamma, \mathrm{H} \delta$, and the $\mathrm{H}$ and $\mathrm{K}$ lines. The green chip (CCD2) covers the range 538-689 nm (15 orders) and includes $\mathrm{H} \alpha$-for the last few orders there are small gaps in the spectrum. And the red chip (CCD3) covers the range $698-836 \mathrm{~nm}$ ( 9 orders), with between-order gaps ranging from $2-5 \mathrm{~nm}$, the largest gaps occurring at the longest wavelengths (e.g., at $734-736 \mathrm{~nm}, 749-752 \mathrm{~nm}, 799-803 \mathrm{~nm}$, and 817-822 nm). The largest gaps in the spectral coverage occur between the chips $(9.5 \mathrm{~nm}$ between CCD1 and CCD2, and $9 \mathrm{~nm}$ between CCD2 and CCD3).

17 MAKEE was written by T.A. Barlow specifically for the reduction of HIRES spectra, and is publicly available at www.astro.caltech.edu/ tb/makee.
}

giant stars in the very metal-poor globular cluster M92 (mean $[\mathrm{Fe} / \mathrm{H}]=-2.33 \mathrm{dex}$, with little within-cluster variation; Cohen 2011). All of the RR Lyr standard stars are RRab pulsators and include RR Lyrae itself ( $K p=7.862$ ), which happens to be located in the Kepler field and has been the subject of several recent detailed investigations (K10; K11; Benedict et al. 2011). The M92 stars served primarily as RV standards and the bright RR Lyr stars as metallicity standards. The most metal poor and metal rich standard stars are X Ari and SW And, respectively, for which $[\mathrm{Fe} / \mathrm{H}]=-2.74 \pm 0.09$ dex and $+0.20 \pm 0.08 \mathrm{dex}$ (see Table 7), and the corresponding Kepler-field RR Lyr stars are NR Lyr and V784 Cyg, for which $[\mathrm{Fe} / \mathrm{H}]=-2.54 \pm 0.11 \mathrm{dex}$ and $-0.05 \pm 0.10$ dex. Although X Ari and NR Lyr are very metal poor, and have metallicities similar to those of the most metal-poor RR Lyr stars known in the LMCs and SMCs (see Haschke et al. 2012), they are not the most metal poor stars known in our Galaxy (see Schörck et al. 2009).

Sample Keck spectra are plotted in Figure 7, for the same wavelength range $(515-520 \mathrm{~nm})$ as Figure 6. The three panels, each of which includes the high $\mathrm{S} / \mathrm{N}$ spectrum of RR Lyrae, show program stars more metal-poor than RR Lyrae (Figure 7(a)), program stars more metal-rich than RR Lyrae (Figure 7(b)), and Keck RR Lyr standard stars (Figure 7(c)). The high-resolution, $\mathrm{S} / \mathrm{N} \sim 1000$, solar spectrum supplied with VWA (Figure 7(c)) was acquired with the KPNO Fourier Transform Spectrometer (Kurucz et al. 1984; Hinkle et al. 2000).

Typical FWHM values were measured for individual and coadded Keck spectra using the same three Fe II spectral lines that were used for the CFHT spectra. The measured FWHM values for the individual spectra (see Table 5) and for the co-added spectra (see Table 7) range from 150 to $470 \mathrm{~m} \AA$, which is similar to the range for the CFHT spectra. As expected the measured FWHM values tend to be largest for phases away from maximum radius. This effect is illustrated in the lower panel of Figure 5, where the FWHM for the three Fe II lines near $450 \mathrm{~nm}$ and for the Ti II line at $450.1 \mathrm{~nm}$ are plotted as a function of the pulsation phase for the nine bright RR Lyr standard stars. The narrowest lines are seen to occur at the earliest phases $(\sim 0.3-0.4)$, and at all phases the Fe II lines (solid symbols) are significantly narrower than the Ti II line (crosses). The same pattern for these four lines is also seen for the Kepler stars (where phase range and scatter are larger), for RR Lyrae (Figure 4 of K10), and for XZ Apr (Figure 18 of For et al. 2011). 
Table 5

Keck-I 10 m HIRES Spectra of Program and Standard Stars

\begin{tabular}{|c|c|c|c|c|c|c|c|c|}
\hline Star & $\begin{array}{l}\text { Spec. } \\
\text { No. } \\
(2)\end{array}$ & $\begin{array}{l}t_{\exp } \\
(\mathrm{s}) \\
(3)\end{array}$ & $\begin{array}{l}\mathrm{S} / \mathrm{N} \\
(4)\end{array}$ & $\begin{array}{l}\text { FWHM } \\
(\mathrm{m} \AA) \\
(5)\end{array}$ & $\begin{array}{l}\text { AM } \\
\text { (6) }\end{array}$ & $\begin{array}{c}\text { HJD (Mid) } \\
2,400,000+ \\
(7)\end{array}$ & $\begin{array}{c}\phi_{\text {puls }} \\
(8)\end{array}$ & $\begin{array}{c}\mathrm{RV} \text { (fxcor) } \\
\left(\mathrm{km} \mathrm{s}^{-1}\right) \\
(9)\end{array}$ \\
\hline \multicolumn{9}{|c|}{ (a) 22 Kepler-field RR Lyrae program stars (excluding RR Lyrae) } \\
\hline \multirow[t]{2}{*}{ V839 Cyg } & 5951 & 1200 & 51 & $379(01)$ & 1.40 & 55778.7493 & 0.100 & $-97.48(14)$ \\
\hline & 5952 & 1200 & 50 & $368(02)$ & 1.30 & 55778.7639 & 0.133 & $-93.78(14)$ \\
\hline \multirow{3}{*}{ V360 Lyr } & 5953 & 1200 & 17 & $348(11)$ & 1.24 & 55778.7786 & 0.495 & $-170.80(26)$ \\
\hline & 5954 & 1200 & 18 & $353(04)$ & 1.20 & 55778.7931 & 0.521 & $-168.85(14)$ \\
\hline & 5955 & 1200 & 19 & 343 (15) & 1.16 & 55778.8076 & 0.547 & $-167.95(17)$ \\
\hline \multirow[t]{3}{*}{ V354 Lyr } & 5957 & 1200 & 20 & $272(05)$ & 1.08 & 55778.8347 & 0.354 & $-207.67(14)$ \\
\hline & 5958 & 1200 & 19 & 327 (11) & 1.08 & 55778.8492 & 0.380 & $-205.35(14)$ \\
\hline & 5959 & 1200 & 18 & $282(05)$ & 1.08 & 55778.8637 & 0.405 & $-202.95(17)$ \\
\hline \multirow[t]{3}{*}{ V368 Lyr } & 5960 & 1200 & 15 & $161(08)$ & 1.09 & 55778.8786 & 0.401 & $-267.41(14)$ \\
\hline & 5961 & 1200 & 11 & $195(11)$ & 1.11 & 55778.8931 & 0.432 & $-264.59(22)$ \\
\hline & 5962 & 1200 & 12 & $201(13)$ & 1.18 & 55778.9076 & 0.464 & $-261.43(99)$ \\
\hline \multirow{6}{*}{ V353 Lyr } & 5963 & 1200 & 9 & $199(18)$ & 1.18 & 55778.9220 & 0.415 & $-199.42(53)$ \\
\hline & 5964 & 1200 & 8 & 145 (17) & 1.22 & 55778.9366 & 0.443 & $-196.91(21)$ \\
\hline & 5965 & 1200 & 7 & $204(51)$ & 1.27 & 55778.9510 & 0.467 & $-194.38(29)$ \\
\hline & 5966 & 1200 & 7 & $\ldots$ & 1.33 & 55778.9656 & 0.493 & $-192.30(17)$ \\
\hline & 6000 & 1200 & 13 & $192(07)$ & 1.39 & 55779.9752 & 0.307 & $-210.26(11)$ \\
\hline & 6001 & 1200 & 12 & $219(35)$ & 1.48 & 55779.9897 & 0.332 & $-207.77(11)$ \\
\hline \multirow[t]{2}{*}{ V782 Cyg } & 5967 & 1200 & 22 & 277 (19) & 1.19 & 55778.9814 & 0.418 & $-60.97(15)$ \\
\hline & 5968 & 1200 & 22 & $287(06)$ & 1.24 & 55778.9962 & 0.446 & $-58.88(14)$ \\
\hline \multirow[t]{2}{*}{ V784 Cyg } & 5969 & 1200 & 19 & 306 (13) & 1.31 & 55779.0128 & 0.462 & $-10.31(17)$ \\
\hline & 5970 & 1200 & 19 & 304 (09) & 1.39 & 55779.0273 & 0.489 & $-8.81(16)$ \\
\hline \multirow[t]{2}{*}{ V1107 Cyg } & 5983 & 1200 & 15 & $215(20)$ & 1.46 & 55779.7425 & 0.475 & $-117.07(12)$ \\
\hline & 5984 & 800 & 14 & $221(27)$ & 1.38 & 55779.7549 & 0.497 & $-115.08(23)$ \\
\hline \multirow[t]{4}{*}{ KIC 9973633} & 5985 & 1200 & 8 & $\ldots$ & 1.47 & 55779.7679 & 0.576 & $-206.84(48)$ \\
\hline & 5986 & 1200 & 8 & $\ldots$ & 1.38 & 55779.7837 & 0.606 & $-203.70(23)$ \\
\hline & 5987 & 1200 & 8 & $\ldots$ & 1.31 & 55779.7982 & 0.635 & $-202.93(35)$ \\
\hline & 5988 & 1200 & 9 & $\ldots$ & 1.26 & 55779.8127 & 0.664 & $-205.54(32)$ \\
\hline KIC 4064484 & 5990 & 800 & 37 & $288(11)$ & 1.10 & 55779.8324 & 0.586 & $-290.79(12)$ \\
\hline \multirow[t]{3}{*}{ KIC 9658012} & 5991 & 1200 & 21 & $369(31)$ & 1.15 & 55779.8457 & 0.499 & $-312.52(10)$ \\
\hline & 5992 & 1200 & 20 & $403(29)$ & 1.13 & 55779.8602 & 0.527 & $-310.41(09)$ \\
\hline & 5993 & 1200 & 21 & $423(09)$ & 1.12 & 55779.8748 & 0.554 & $-309.32(11)$ \\
\hline \multirow[t]{3}{*}{ V445 Lyr } & 5996 & 1200 & 14 & $319(24)$ & 1.13 & 55779.9167 & 0.285 & $-392.79(24)$ \\
\hline & 5997 & 1200 & 14 & $237(25)$ & 1.17 & 55779.9312 & 0.314 & $-390.40(17)$ \\
\hline & 5998 & 1200 & 14 & $245(21)$ & 1.21 & 55779.9457 & 0.342 & $-388.18(11)$ \\
\hline V1104 Cyg & 5999 & 1000 & 37 & $174(03)$ & 1.31 & 55779.9599 & 0.420 & $-293.96(10)$ \\
\hline KIC 5520878 & 6002 & 1000 & 45 & $319(02)$ & 1.47 & 55780.0041 & 0.459 & $-0.70(29)$ \\
\hline \multirow[t]{2}{*}{ KIC 9717032} & 6003 & 1200 & 13 & 303 (19) & 1.46 & 55780.0179 & 0.321 & $-457.11(21)$ \\
\hline & 6004 & 1200 & 14 & $281(31)$ & 1.57 & 55780.0324 & 0.347 & $-454.63(11)$ \\
\hline V450 Lyr & 6017 & 900 & 11 & $273(42)$ & 1.37 & 55780.7406 & 0.546 & $-269.48(19)$ \\
\hline & 6018 & 1200 & 13 & $240(07)$ & 1.32 & 55780.7535 & 0.571 & $-267.65(10)$ \\
\hline & 6019 & 1200 & 16 & $254(10)$ & 1.25 & 55780.7703 & 0.606 & $-265.84(10)$ \\
\hline V366 Lyr & 6022 & 1200 & 15 & $301(01)$ & 1.13 & 55780.8367 & 0.658 & $-68.51(16)$ \\
\hline & 6023 & 1200 & 16 & 357 (05) & 1.12 & 55780.8512 & 0.685 & $-68.47(18)$ \\
\hline & 6024 & 1200 & 16 & $350(28)$ & 1.12 & 55780.8657 & 0.712 & $-67.61(18)$ \\
\hline V346 Lyr & 6027 & 1200 & 10 & $293(08)$ & 1.18 & 55780.9138 & 0.619 & $-272.88(14)$ \\
\hline V808 Cyg & 6028 & 1200 & 19 & $473(16)$ & 1.10 & 55780.9338 & 0.630 & $+32.40(15)$ \\
\hline & 6029 & 600 & 4 & $\ldots$ & 1.12 & 55780.9449 & 0.649 & $+31.83(33)$ \\
\hline V350 Lyr & 6032 & 1200 & 22 & $283(25)$ & 1.44 & 55780.9775 & 0.523 & $-91.41(14)$ \\
\hline & 6033 & 1200 & 23 & 324 (17) & 1.54 & 55780.9920 & 0.547 & $-89.56(15)$ \\
\hline V2178 Cyg & 6034 & 1200 & 26 & $212(13)$ & 1.36 & 55781.0085 & 0.241 & $-116.89(20)$ \\
\hline & 6035 & 1200 & 26 & $213(09)$ & 1.46 & 55781.0230 & 0.270 & $-115.04(16)$ \\
\hline V715 Cyg & 6036 & 1200 & 15 & 238 (17) & 1.57 & 55781.0387 & 0.494 & $-78.36(18)$ \\
\hline & 6037 & 1200 & 15 & $229(09)$ & 1.73 & 55781.0532 & 0.525 & $-75.87(20)$ \\
\hline & & & ) Nine & R Lyrae sta & $\operatorname{ling} \mathrm{RI}$ & & & \\
\hline ST Boo & 5950 & 600 & 160 & $198(02)$ & 1.04 & 55778.7289 & 0.359 & $1.23(10)$ \\
\hline V445 Oph & 5956 & 600 & 125 & $315(05)$ & 1.24 & 55778.8211 & 0.436 & $-11.96(13)$ \\
\hline RR Lyr & 5971 & 200 & 324 & $276(06)$ & 1.68 & 55779.0361 & 0.535 & $-56.48(06)$ \\
\hline SW And & 5972 & 300 & 184 & $367(06)$ & 1.04 & 55779.0416 & 0.557 & $-4.32(07)$ \\
\hline X Ari & 5973 & 300 & 168 & $188(05)$ & 1.63 & 55779.0441 & 0.310 & $-42.01(13)$ \\
\hline VX Her & 5982 & 200 & 109 & $202(02)$ & 1.01 & 55779.7287 & 0.318 & $-381.72(20)$ \\
\hline VY Ser & 5989 & 300 & 135 & $232(03)$ & 1.40 & 55779.8218 & 0.449 & $-137.41(10)$ \\
\hline
\end{tabular}


Table 5

(Continued)

\begin{tabular}{|c|c|c|c|c|c|c|c|c|}
\hline Star & $\begin{array}{l}\text { Spec. } \\
\text { No. } \\
(2)\end{array}$ & $\begin{array}{l}t_{\exp } \\
(\mathrm{s}) \\
(3)\end{array}$ & $\begin{array}{l}\mathrm{S} / \mathrm{N} \\
(4)\end{array}$ & $\begin{array}{c}\text { FWHM } \\
(\mathrm{m} \AA) \\
(5)\end{array}$ & $\begin{array}{l}\text { AM } \\
(6)\end{array}$ & $\begin{array}{l}\text { HJD (Mid) } \\
2,400,000+ \\
\quad(7)\end{array}$ & $\begin{array}{c}\phi_{\text {puls }} \\
(8)\end{array}$ & $\begin{array}{c}\mathrm{RV} \text { (fxcor) } \\
\left(\mathrm{km} \mathrm{s}^{-1}\right) \\
(9)\end{array}$ \\
\hline UU Cet & 6006 & 300 & 74 & $226(03)$ & 1.25 & 55780.0649 & 0.379 & $-112.28(12)$ \\
\hline RR Cet & 6042 & 300 & 226 & $212(03)$ & 1.06 & 55781.1104 & 0.365 & $-77.06(09)$ \\
\hline \multicolumn{9}{|c|}{ (c) Three red giants in the globular cluster M92 } \\
\hline \multirow[t]{2}{*}{ M92-XII-34 } & 5994 & 1200 & 77 & $178(02)$ & 1.28 & 55779.8894 & $\ldots$ & $-113.86(26)$ \\
\hline & 5995 & 800 & 59 & $173(03)$ & 1.35 & 55779.9017 & $\ldots$ & $-114.23(26)$ \\
\hline \multirow[t]{2}{*}{ M92-IV-79 } & 6025 & 1200 & 70 & $158(04)$ & 1.27 & 55780.8828 & $\ldots$ & $-120.74(13)$ \\
\hline & 6026 & 1200 & 59 & $156(05)$ & 1.33 & 55780.8975 & $\ldots$ & $-120.92(13)$ \\
\hline \multirow[t]{2}{*}{ M92-IV-10 } & 6030 & 1200 & 41 & $171(06)$ & 1.78 & 55780.9545 & $\ldots$ & $-120.31(19)$ \\
\hline & 6031 & 500 & 39 & $182(12)$ & 1.98 & 55780.9650 & $\ldots$ & $-120.04(19)$ \\
\hline
\end{tabular}

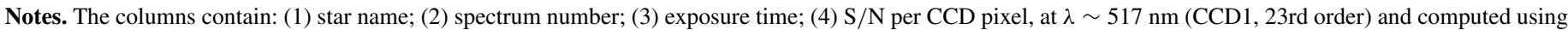

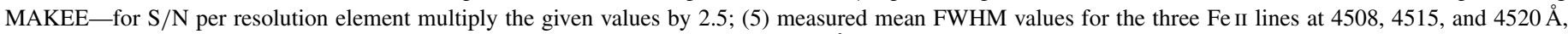

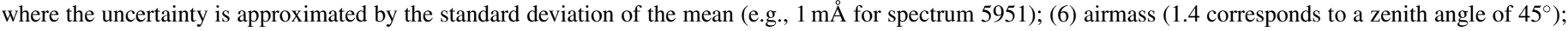

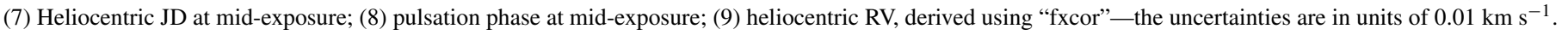

\subsection{Radial Velocities}

It is well known that the RVs derived from spectra of RR Lyr stars are phase dependent and are not necessarily the same as the velocities that the stars would have if they were not pulsating (Oke 1966; Liu \& Janes 1989, 1990a, 1990b). Furthermore, the derived RVs depend on the spectral lines used for their measurement: RV curves calculated with metal lines tend to have smaller amplitudes than those calculated using Balmerseries lines $(\mathrm{H} \alpha, \mathrm{H} \beta, \mathrm{H} \gamma)$-a difference that has recently been quantified by Sesar (2012). A nice illustration of both effects is given in Figure 2 of Preston (2011), which shows multiple RV curves for the southern RRab stars Z Mic and RV Oct.

In general, the RVs of RR Lyr stars are most negative near maximum light (i.e., at $\phi_{\text {puls }} \sim 0$ ) and most positive near minimum light (which, depending on the risetime, occurs for RRab stars around $\phi_{\text {puls }} \sim 0.80-0.95$ and at earlier phases for RRc stars). Fundamental-mode pulsators also tend to show larger RV variations than first-overtone pulsators (as is also the case for the light variations), with double-mode (RRd) and other multi-mode pulsators showing the most complex variations. If a star exhibits the Blazhko effect then the RV curve will reflect the amplitude and phase variations of such stars, and if pulsational velocities are required (e.g., Baade-Wesselink studies) then the observed RV must be corrected for projection effects (see Nardetto et al. 2004, 2009).

For our spectra the heliocentric RVs given in Tables 4 and 5 were derived using metal lines and the IRAF "fxcor" routine. The heliocentric corrections were made during standard pipeline reductions, by Upena for the CFHT data and by MAKEE for the Keck data. For the ESPaDOnS spectra only the limited wavelength range 511-518 nm was used for the crosscorrelations and a typical RV uncertainty is $\sim \pm 0.2 \mathrm{~km} \mathrm{~s}^{-1}$. The HIRES flux spectra were normalized using MAKEE and very accurate pixel shifts were calculated by cross-correlating all pairs of spectra that were taken over the three night run. The results were used to derive very precise and accurate relative velocities that were then shifted to the mean of the RVs computed relative to the known wavelengths.

The derived heliocentric RVs for the three M92 red giant standard stars (see Table 5) are all close to the cluster mean of $-120 \mathrm{~km} \mathrm{~s}^{-1}$, suggesting that all are cluster members (see Roederer \& Sneden 2011; Cohen 2011). Comparison with the
RVs derived by Drukier et al. (2007) gives a median difference of $\sim 0.5 \mathrm{~km} \mathrm{~s}^{-1}$ and an rms difference of $0.2 \mathrm{~km} \mathrm{~s}^{-1}$.

The $\gamma$-velocities for the nine bright RR Lyrae standard stars were computed using Liu's RV template and are given in Table 6. The last column contains the differences between our estimates and those of Layden (1994), where the mean difference is $1.3 \mathrm{~km} \mathrm{~s}^{-1}$.

\section{4. $[\mathrm{Fe} / \mathrm{H}]$ and Atmospheric Parameters}

Estimation of metal abundances and other atmospheric parameters from high dispersion spectra depends on numerous assumptions regarding the stellar atmosphere where the spectral lines were formed. In our analyses, which were performed using the VWA, MOOG, and Spectroscopy Made Easy (SME) packages, the lines were assumed to form in a one-dimensional plane-parallel atmosphere under local thermodynamic equilibrium (LTE) conditions. For RR Lyr stars (absolute magnitudes $\sim 0.5$ ) the atmospheric extensions are sufficiently small (less than $1 \%$ according to Figure 1 of Heiter \& Eriksson 2006) that the plane-parallel assumption was judged to be adequate for the derivation of iron-to-hydrogen ratios.

The observed Kepler-field RR Lyr stars are a mixture of halo and old-disk stars, with distances ranging from $265 \pm 11 \mathrm{pc}$ for RR Lyrae (Benedict et al. 2011) to $\sim 25 \mathrm{kpc}$ for the faintest stars (N11); therefore they are expected to be old and to have a wide range of chemical and kinematic characteristics. In the $\mathrm{H}-\mathrm{R}$ diagram they are horizontal branch stars at various stages of evolution away from the ZAHB, and over a pulsation cycle $L$ and $T_{\text {eff }}$ trace out a loop in the instability strip. The masses are expected to be in the range $0.5-0.8 \mathcal{M}_{\odot}$, and most of the stars are expected to be near the ZAHB-level appropriate for the mass and metal abundance of the star (see Figure 13 of N11).

The Blazhko and non-Blazhko RR Lyr stars in the Kepler field occur in approximately equal proportions. The pulsations of the non-Blazhko stars are mainly fundamental or first-overtone radial modes. To date no "classical" double-mode RR Lyrae stars (i.e., RRd stars) pulsating simultaneously in both modes have been identified; however, Molnár et al. (2012) recently detected a very weak first-overtone pulsation in RR Lyrae. When averaged over a pulsation cycle the mean values of $T_{\text {eff }}$ are expected to range from 6000-7500 K, with the ab-type stars having cooler mean surface temperatures than the c-type stars. The ranges 


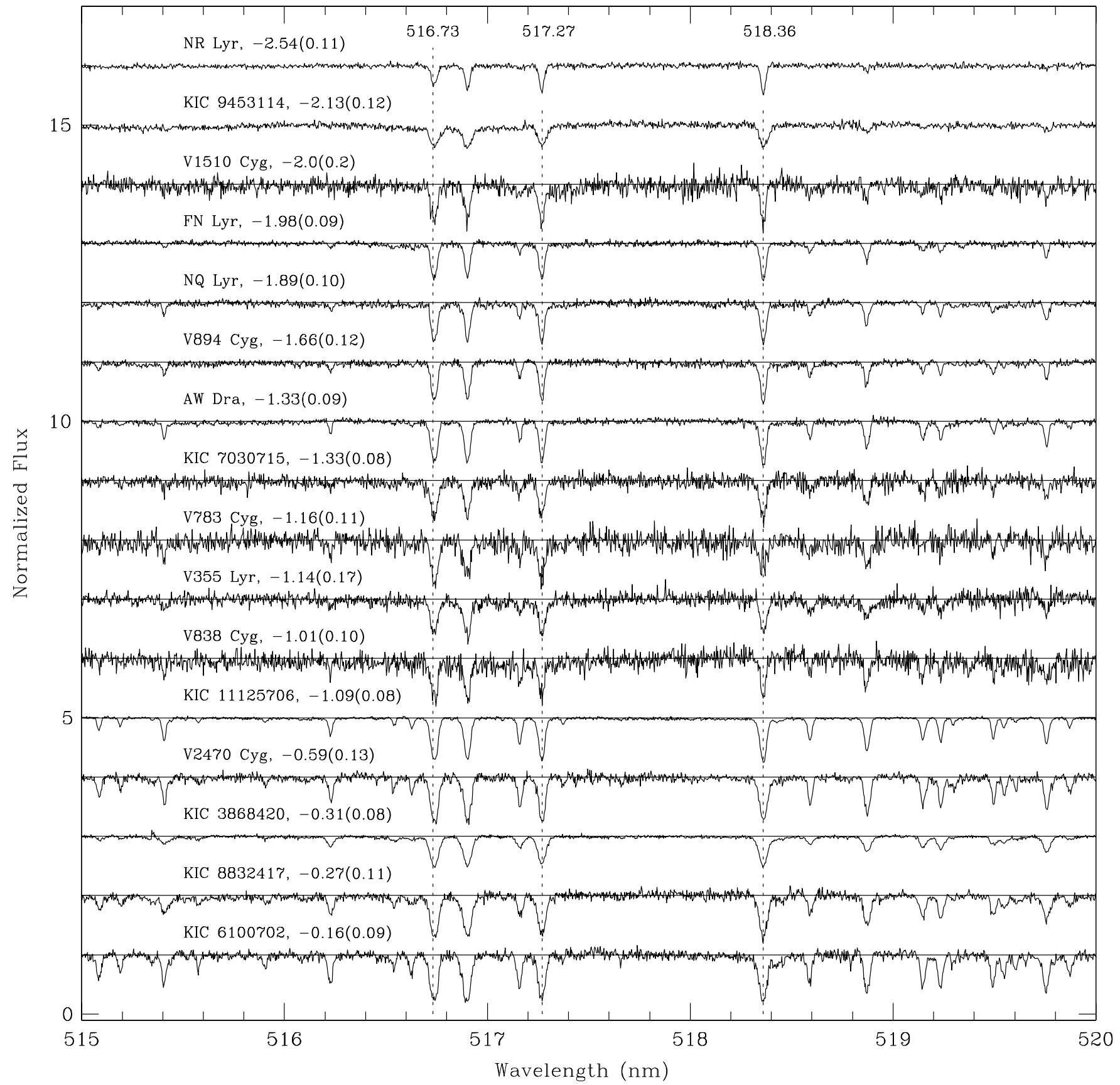

Figure 6. Mosaic showing the $517 \mathrm{~nm} \mathrm{Mg}$ I triplet region of the CFHT spectra for the 16 stars for which metallicities could be derived. The rest wavelengths of the $\mathrm{Mg}$ I triplet lines are given at the top of the graph. The enhanced line strengths of the most metal-rich stars (bottom) clearly distinguish them from the more metal-poor stars (top). The probable HADS star, KIC 3868420, is third from the bottom.

of the mean values of $\log g$ and $L$ are expected to be 2-3 and $40-60 L_{\odot}$. The instantaneous values for these quantities, which are phase dependent, span much wider ranges-see, for example, Jurcsik et al. (2009a, 2009b, 2009c), who suggested that the $T_{\text {eff }}$ range might be as large as $3500 \mathrm{~K}(5500-9000 \mathrm{~K})$, the range of $\log g \sim 1.5$ to 4 , and the range of $L \sim 30-90 L_{\odot}$. For all three physical characteristics the maxima occur slightly before $\phi_{\text {puls }}=1$. Because the CFHT and Keck spectra were taken at pulsation phases away from maximum light the RRab stars were expected to have "instantaneous" $T_{\text {eff }}$ values in the considerably smaller range 6000-6500 K (see Figures 7-10, 36 and 37 of For et al. 2011), with $\log g$ between 2 and 3. An exception is V839 Cyg, which was observed just after maximum light, at $\phi_{\text {puls }}=0.12$, and therefore expected to have a rather higher temperature. Unlike solar-like stars which typically have a microturbulent parameter $\xi_{t} \sim 1 \mathrm{~km} \mathrm{~s}^{-1}$, the $\xi_{t}$ for RR Lyr stars generally range from 2.5 to $4.0 \mathrm{~km} \mathrm{~s}^{-1}$, with instantaneous values at maximum radius tending to occur at the lower end of this range (i.e., $\xi_{t} \sim 2.5-3.0 \mathrm{~km} \mathrm{~s}^{-1}$ for phases between 0.2 and 0.6 - see Figures 13, 14, and 17 of For et al. 2011). Other information is available for some of the brighter stars. For example, for RR Lyrae at $\phi_{\text {puls }}=0.54$ the $\log g$ and $T_{\text {eff }}$ are expected to be $\sim 2.75$ and $6250 \pm 100 \mathrm{~K}$ (see Table 5.2 of Kolenberg 2002), both of which vary from one Blazhko cycle to the next.

For the solar photosphere the abundance of iron relative to the number of hydrogen atoms per unit volume was assumed to be $\log \epsilon(\mathrm{Fe})=7.50$, where $\log \epsilon(\mathrm{Fe}) \equiv \log \left(N_{\mathrm{Fe}} / N_{\mathrm{H}}\right)+12.0$ and 


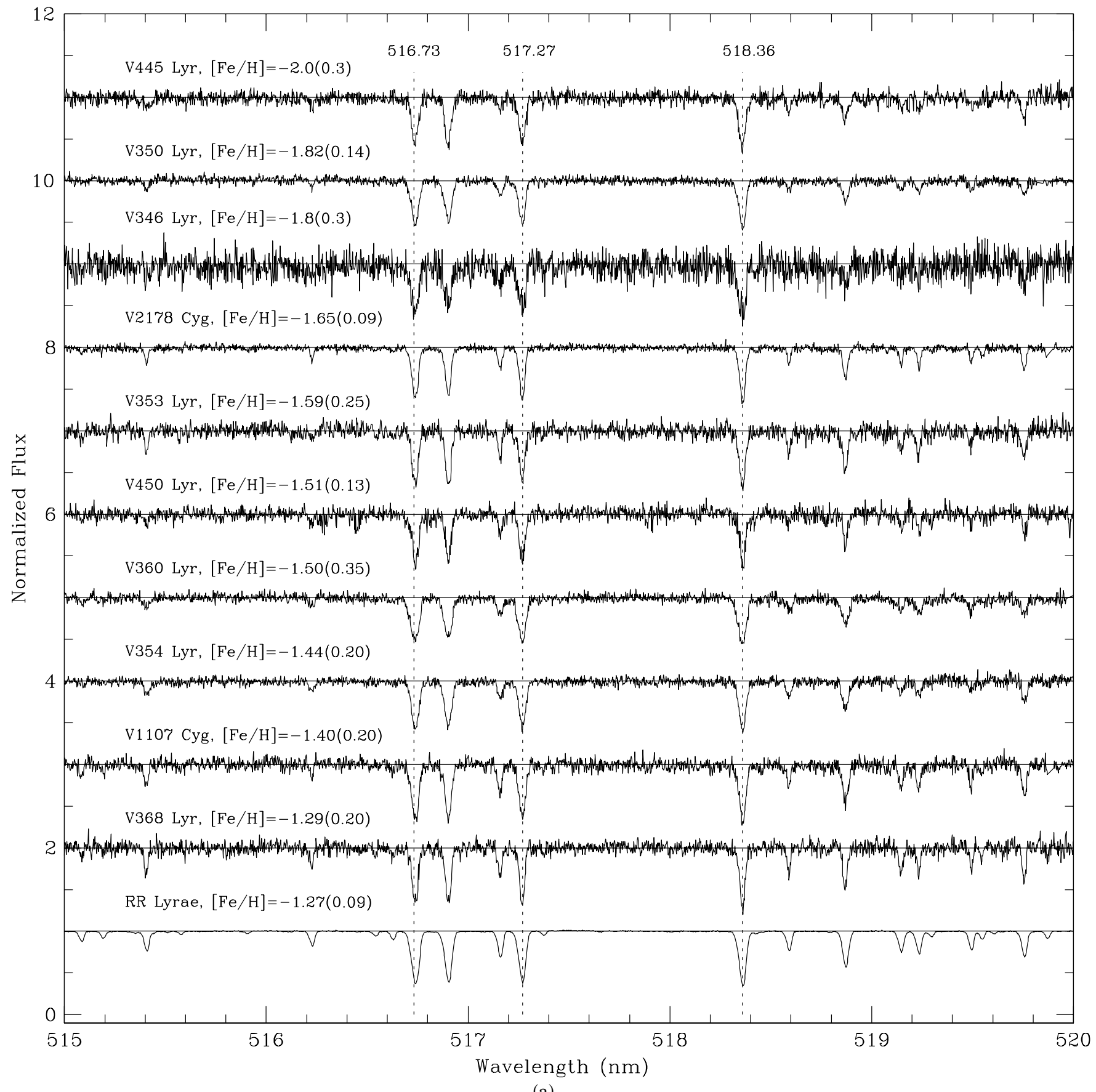

(a)

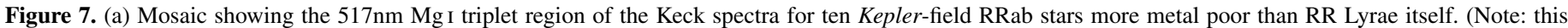

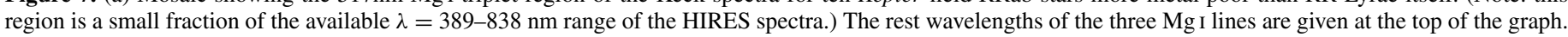

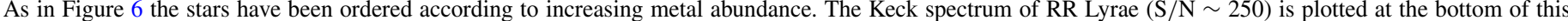

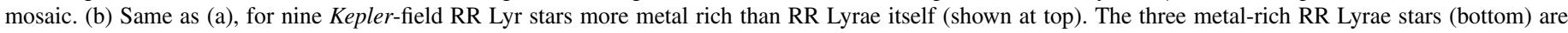

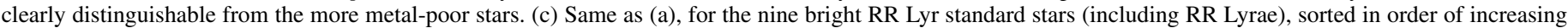
metal abundance, and for the Sun. The broad Mg I lines in the solar spectrum readily show the greater pressure broadening in the Sun than in the RR Lyr stars.

$\log N_{\mathrm{H}}=12.0$. This value is consistent with the iron abundance of $7.45 \pm 0.08$ recommended by Holweger (2001), the value of 7.52 adopted by MOOG (Sneden 2002), and with the most recent value of $7.50 \pm 0.04$ given in the review by Asplund et al. (2009). For iron abundances relative to hydrogen and relative to the Sun we use $[\mathrm{Fe} / \mathrm{H}]_{*}=\log \left(N_{\mathrm{Fe}} / N_{\mathrm{H}}\right)_{*}-\log \left(N_{\mathrm{Fe}} / N_{\mathrm{H}}\right)_{\text {solar }}$. When abundances are expressed relative to the total number of atoms per unit volume (e.g., the values output from VWA and Kurucz's ATLAS9 program), the photospheric "abundance" of Fe is denoted $A_{\mathrm{Fe}} \equiv \log \left(N_{\mathrm{Fe}} / N_{\text {total }}\right)$, where $N_{\mathrm{Fe}}$ is the number of Fe atoms per unit volume and $N_{\mathrm{Fe}} / N_{\text {total }}$ is the corresponding fraction of $\mathrm{Fe}$ relative to the total number density of atoms. If the number densities for hydrogen and helium are assumed to be $\log N_{\mathrm{H}}=12.0$ and $\log N_{\mathrm{He}}=10.99$ then these two elements are the only significant contributors to $N_{\text {total }}$ and we have $\log N_{\text {total }}=$ 12.04. Thus, the solar number fractions of $\mathrm{H}, \mathrm{He}$, and $\mathrm{Fe}$ are, respectively, $N_{\mathrm{H}} / N_{\text {total }}=91.1 \%, N_{\mathrm{He}} / N_{\text {total }}=8.9 \%$, and $N_{\mathrm{Fe}} /$ $N_{\text {total }}=0.0029 \%$ (i.e., the $\log$ of the Fe abundance relative to the total number density is $\log \left(N_{\mathrm{Fe}} / N_{\mathrm{tot}}\right)=-4.54$, which follows from $\left.A_{\mathrm{Fe}}=\log \epsilon(\mathrm{Fe})-\log N_{\text {total }}=7.50-12.04\right)$. 


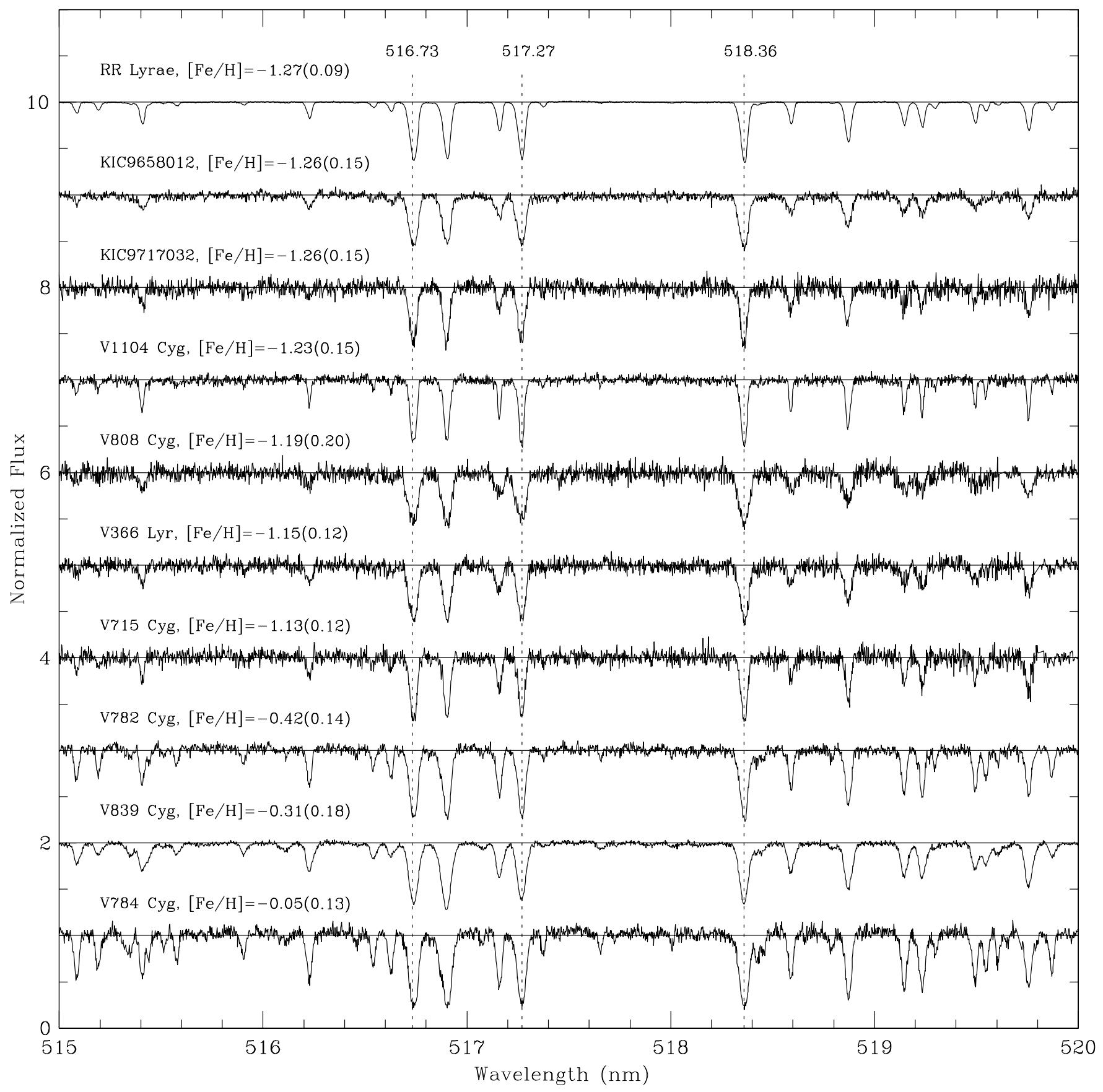

(b)

Figure 7. (Continued)

\subsubsection{Parameter Estimation with VWA and MOOG}

The bulk of the spectroscopic data analyses of both the CFHT and Keck data was carried out with the spectral synthesis program VWA ${ }^{18}$ (Bruntt et al. 2002, 2008, 2010a, 2010b). This all-purpose program has the capability of normalizing and co-adding spectra, computing synthetic spectra, selecting suitable lines for abundance determination, measuring EWs, calculating expected EWs for the assumed model parameters, and calculating abundances for a wide range of elements/

\footnotetext{
18 The VWA software was written by Hans Bruntt (University of Aarhus, Denmark), in IDL, and is publicly available at https://sites.google.com/site/vikingpowersoftware/.
}

ions. The abundances follow from iteratively varying the model parameters, calculating synthetic spectra, and then comparing the EWs calculated from the spectra with the measured EWs.

For each RR Lyrae star the following photospheric parameters were derived: the effective temperature, $T_{\text {eff }}(\mathrm{K})$; the surface gravity, $\log g\left(\mathrm{~cm} \mathrm{~s}^{-2}\right)$; the microturbulent velocity, $\xi_{t}\left(\mathrm{~km} \mathrm{~s}^{-1}\right)$; the macroturbulent velocity, $v_{\text {mac }}$; the projected rotational velocity, $v \sin i$; and the spectroscopic iron-to-hydrogen ratio, $[\mathrm{Fe} / \mathrm{H}]_{\text {spec }}$. Because the RR Lyr stars are undergoing (mainly) radial pulsations, these parameters are "instantaneous" quantities, specific for the times (phases) at which the spectroscopic observations were made. The metal abundances, while depending on the assumed atmospheric conditions, should be independent 


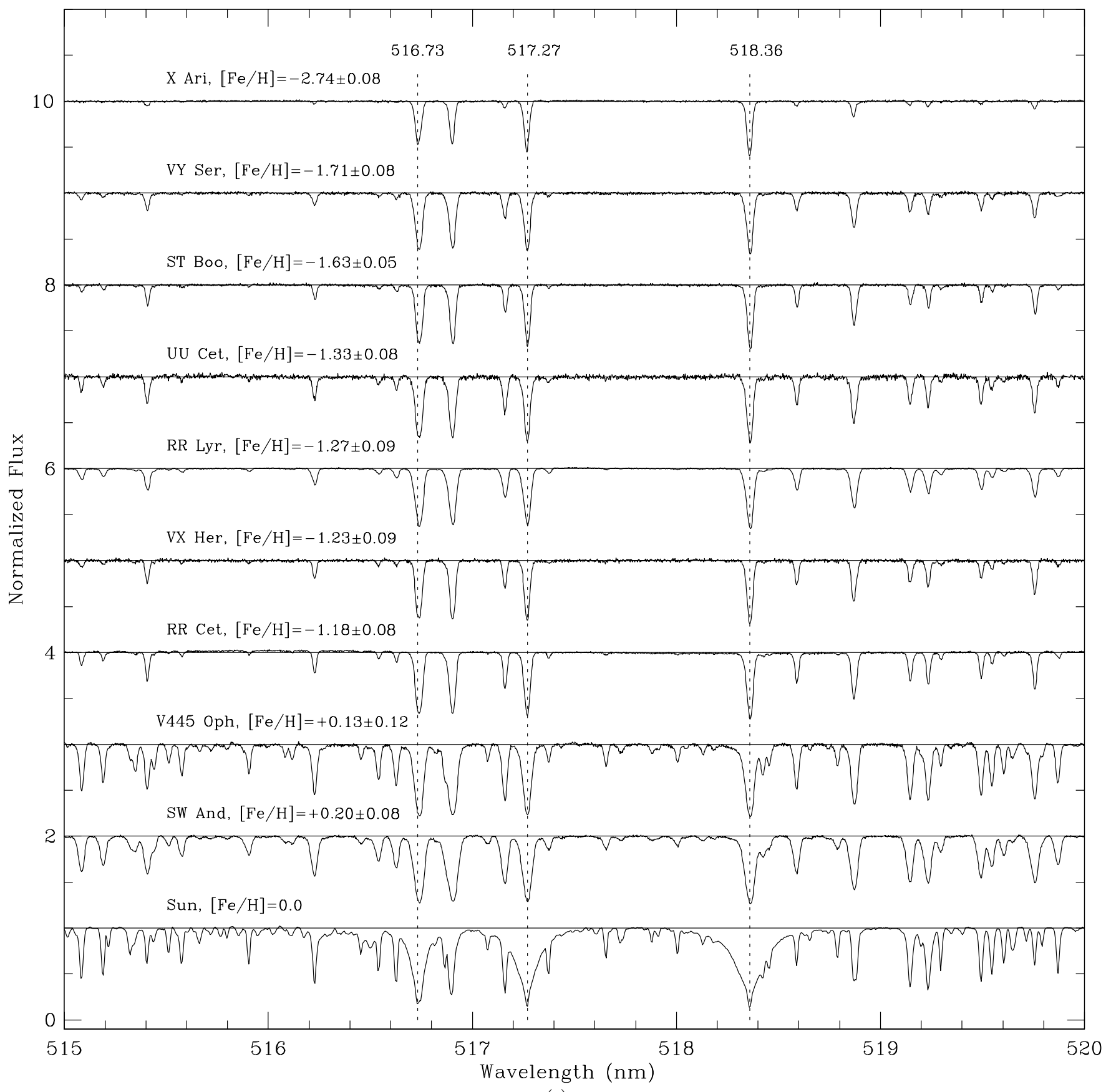

(c)

Figure 7. (Continued)

of the observed pulsation phases (see Figures 13 and 14 of For et al. 2011, which show the variation with pulsation phase of their derived $T_{\text {eff }}, \log g,[\mathrm{Fe} / \mathrm{H}]$ and $\xi_{t}$ values).

The synthetic spectra were calculated within VWA using the SYNTH code (Piskunov 1992; Valenti \& Piskunov 1996), with atomic parameters (e.g., $\log g f$ values) and line-broadening coefficients retrieved from the Vienna atomic line database (VALD; Kupka et al. 1999). ${ }^{19}$ Two sets of model stellar atmospheres were used. For all the stars the model atmosphere grid of ATLAS9 models provided by Heiter et al. (2002) was used. For this grid the step-increments in effective temperature, surface gravity, and metal abundance are, respectively,

\footnotetext{
19 See http://www.astro.uu.se/ vald/php/vald.php
}

$\Delta T_{\text {eff }}=250 \mathrm{~K}, \Delta \log g=0.5$, and $\Delta[\mathrm{Fe} / \mathrm{H}]=0.25$ dex. For the bright RR Lyrae stars both ATLAS9 and MARCS models were used.

The derivation of $[\mathrm{Fe} / \mathrm{H}]_{\text {spec }}$ values followed from analysis of the normalized flux spectra. For each star an initial set of values for the atmospheric parameters was assumed, and a synthetic spectrum was calculated. The usual starting values were: $T_{\text {eff }}=6500 \mathrm{~K}, \log g=2.50, \xi_{t}=2.50 \mathrm{~km} \mathrm{~s}^{-1}$ and $v_{\text {mac }}=2 \mathrm{~km} \mathrm{~s}^{-1}$. After adjusting the projected rotational velocity, $v \sin i$ and the resolving power $(R=65,000$ for the CFHT spectra and 36,000 for the Keck spectra) for each analyzed spectrum, the spectrum was Doppler shifted according to the RV (see Tables 4 and 5) and aligned with the synthetic template spectrum. 
Table 6

$\gamma$-velocities for the RR Lyr Standard Stars

\begin{tabular}{lcccc}
\hline \hline Star & \multirow{2}{*}{$\phi_{\text {puls }}$} & \multicolumn{3}{c}{$\gamma$-velocity $\left(\mathrm{km} \mathrm{s}^{-1}\right)$} \\
\cline { 2 - 5 } & & Layden & This Paper & Diff. \\
$(1)$ & $(2)$ & $(3)$ & $(4)$ & $(5)$ \\
\hline ST Boo & 0.359 & $+13 \pm 4$ & $+1.7 \pm 3$ & $11 \pm 5$ \\
V445 Oph & 0.436 & $-22 \pm 5$ & $-22.6 \pm 4$ & $1 \pm 6$ \\
RR Lyr & 0.535 & $-63 \pm 8$ & $-72.0 \pm 2$ & $9 \pm 8$ \\
SW And & 0.557 & $-21 \pm 2$ & $-14.1 \pm 3$ & $7 \pm 4$ \\
X Ari & 0.310 & $-35 \pm 3$ & $-41.6 \pm 3$ & $-7 \pm 4$ \\
VX Her & 0.318 & $-375 \pm 40$ & $-361.5 \pm 5$ & $-14 \pm 40$ \\
VY Ser & 0.449 & $-145 \pm 1$ & $-142.8 \pm 3$ & $-2 \pm 3$ \\
UU Cet & 0.379 & $-116 \pm 20$ & $-110.3 \pm 5$ & $-6 \pm 21$ \\
RR Cet & 0.365 & $-75 \pm 1$ & $-74.4 \pm 3$ & $-1 \pm 3$ \\
\hline
\end{tabular}

Notes. The columns contain: (1) star name; (2) pulsation phase at the midtime of the spectrum; (3) the $\gamma$-velocity from L94; (4) the $\gamma$-velocity from the present paper; and (5) the $\gamma$-velocity difference, in the sense Layden minus this paper. Note also that RR Lyr, SW And and ST Boo are Blazhko variables with modulation periods of 39.6 days (see K11), 36.8 days (Szeidl 1988; see also Le Borgne et al. 2012), and 284 days (see Le Borgne et al. 2012), respectively.

The final set of fitted lines was inspected and poor fits rejected. The microturbulent velocity, $\xi_{t}$, was determined by requiring that the correlation between the derived Fe abundances and the reduced EWs (i.e., $\mathrm{EW} / \lambda$ ) was near zero; $T_{\text {eff }}$ was estimated by requiring that the correlation of $\mathrm{Fe}$ abundance and the lower excitation potential (EP) was near zero; and the surface gravities were calculated by requiring good agreement between the iron abundances determined from lines of the Fe I and Fe II ionization stages (which depended on the assumed $T_{\text {eff }}$ and $\log g$ values). Although LTE conditions are assumed by VWA, the effect of departures from LTE on the $[\mathrm{Fe} / \mathrm{H}]$ values derived using the $\mathrm{Fe}$ I lines were considered, and where appropriate the corrections calculated by Rentzsch-Holm (1996) were applied; these were usually smaller than $\sim 0.10$ dex. The Fe II lines were assumed to be unaffected by deviations from LTE.

Table 7 summarizes the basic spectroscopic information derived from the VWA analyses of the CFHT and Keck spectra, where the stars are sorted by type (non-Blazhko, Blazhko, RRc, etc.), and within a given type by spectroscopic metal abundance. The pulsation phases (Column 3) are mid-exposure values (i.e., average values of the mid-times when multiple spectra were taken), and the $\mathrm{S} / \mathrm{N}$ per pixel (Column 4) are for the co-added normalized spectra. ${ }^{20}$ The FWHM values (Column 5) are for the co-added spectra and the $T_{\text {eff }}, \log g$, and $\xi_{t}$ values (Column 6) are either those assumed for the adopted VWA runs, or are interpolated values for two similar atmosphere models. The value $v_{\text {mac }}$ was arbitrarily set to either 2 or 3 for all the stars, while the projected rotational velocity, $v \sin i$, was set to $2 \mathrm{~km} \mathrm{~s}^{-1}$ for the Keck spectra and 7-10 $\mathrm{km} \mathrm{s}^{-1}$ for the CFHT spectra; the impact on these choices on the derived abundances seems to have been minimal. The iron-to-hydrogen abundances (Columns 7 and 8) are for the neutral and singly ionized ions, the weighted average of which, $[\mathrm{Fe} / \mathrm{H}]$, is given in Column 9. The derived metallicities range from a low of $-2.54 \pm 0.11 \mathrm{dex}$ (for NR Lyr) to a high of $-0.05 \pm 0.10$ dex (for V784 Cyg). The same two stars were the extremes identified by N11 in their analysis of the Q1-Q5 Kepler photometry of non-Blazhko variables, and the four non-Blazhko stars that were suspected

\footnotetext{
20 A comparison of the MAKEE and "rainbow" S/N values for the seven stars with only single exposures shows the MAKEE values to be systematically larger (by $\sim 16 \%$ ) than the "rainbow" values.
}

(from their locations in Figure 9 of N11) of being metal rich (V782 Cyg, V784 Cyg, KIC 6100702, and V2470 Cyg) are confirmed as such. Not included in Table 7 are four stars for which the VWA analysis of the co-added spectrum proved inconclusive or impossible: (1) V1510 Cyg, a non-Blazhko star $(K p=14.5 \mathrm{mag})$; (2) the faint non-Blazhko star V346 Lyr $(K p=16.4 \mathrm{mag})$; (3) the faint Blazhko star KIC 9973633 $(K p=17.0 \mathrm{mag})$, for which the $\mathrm{S} / \mathrm{N}$ of the co-added Keck spectrum (not plotted in Figure 7) was insufficient for estimating $[\mathrm{Fe} / \mathrm{H}]$; and (4) the faint $(K p=17.4 \mathrm{mag})$ extreme-Blazhko star V445 Lyr (see Guggenberger et al. 2012). In all cases except KIC 9973633 we were able to derive $[\mathrm{M} / \mathrm{H}]$ values using SME (see Table 9).

The EWs derived using VWA are in good agreement with the values measured by hand using the IRAF "splot" routine, with values calculated with ARES, and with the values calculated from the synthetic spectra. The EWs of the standard stars also agree (to within the uncertainties arising from the observations being made at different phases) with the values listed in Table 3(b) of C95. ${ }^{21}$ To minimize the effects of line saturation only $\mathrm{Fe}_{\mathrm{I}}$ and $\mathrm{Fe}$ II lines with $\mathrm{EW}<90 \mathrm{~m} \AA$ were used in our analysis. A table of measured and calculated EWs ( $\mathrm{m} \AA$ ) for the $\mathrm{Fe}_{\mathrm{I}}$ and $\mathrm{Fe}$ II lines (program and standard stars), including the assumed lower EPs and $\log g f$ values (from the VALD database), is available from the first author.

As a check on the VWA results $[\mathrm{Fe} / \mathrm{H}]$ abundances for several of the stars were also derived using the LTE Stellar Line Analysis program MOOG (Sneden 1973, 2002; Sneden et al. 2011). ${ }^{22}$ In this case EWs were calculated using the highly automated program ARES (Sousa et al. 2007, 2008). A comparison of the EWs derived by ARES, by VWA, and by the IRAF "splot" routine, showed excellent agreement for EWs between 5 and $100 \mathrm{~m} \AA$. The WEBMARCS grid of model atmospheres (Gustafsson et al. 1975, 2008) ${ }^{23}$ was used for the MOOG calculations. These models used the Grevesse et al. (2007) compositions for the solar photosphere, and the available grid had $T_{\text {eff }}$ values up to $7000 \mathrm{~K}$ with a step size of $250 \mathrm{~K}$. Unfortunately the smallest surface gravities available were for $\log g=3$, which is larger than the value appropriate for most RR Lyrae stars.

The MOOG results are summarized in Table 8 . The assumed model atmospheric parameters are given in Column 2, where the uncertainties are $\sim \pm 150 \mathrm{~K}$ for $T_{\text {eff }}, \pm 0.2 \mathrm{~cm} \mathrm{~s}^{-2}$ for $\log g$, and $\pm 0.5 \mathrm{~km} \mathrm{~s}^{-1}$ for $\xi_{t}$. Columns 3 and 4 give $\log \epsilon$ values (relative to hydrogen) for the $\mathrm{Fe}$ I and $\mathrm{Fe}$ II ions (standard output from the "ABFIND" routine in MOOG). Also given are the number of lines used to derive the abundance. Column 5 contains the average iron abundance, $\log \epsilon(\mathrm{Fe})$, weighted inversely by the $\mathrm{Fe}$ I and $\mathrm{Fe}$ II uncertainties, and the last column gives $[\mathrm{Fe} / \mathrm{H}]$ values, derived by adding $12.50 \pm 0.05$ to the Column 5 abundances. Since only a limited number of MOOG runs were made the uncertainties are larger than for the VWA results, the largest discrepancies occurring for V782 Cyg and SW And.

\subsubsection{Parameter Estimation with SME}

Atmospheric parameters for the program and standard RR Lyr stars were also derived using the SME program of Valenti

\footnotetext{
21 For RR Lyrae itself the Clementini et al. (1995) EWs are larger than the ARES EWs for lines with EWs $\geqslant 100$; however, such lines are not used here. 22 MOOG is publicly available at www.as.utexas.edu/ chris/moog.html (2010 version).

23 The WEBMARCS models, also known as "MARCS 2008," or "MARCS 35," or "new MARCS" models, are available online at http://marcs.astro.uu.se.
} 
Table 7

Spectroscopic Iron-to-Hydrogen Abundances (VWA) for the Kepler-field RR Lyrae Stars

\begin{tabular}{|c|c|c|c|c|c|c|c|c|}
\hline \multirow{2}{*}{$\begin{array}{l}\text { Star } \\
\text { (1) }\end{array}$} & \multirow{2}{*}{$\begin{array}{l}\text { Obs. } \\
\text { (2) }\end{array}$} & \multirow{2}{*}{$\begin{array}{c}\phi_{\text {puls }} \\
(3)\end{array}$} & \multirow{2}{*}{$\begin{array}{l}\mathrm{S} / \mathrm{N} \\
(4)\end{array}$} & \multirow{2}{*}{$\begin{array}{l}\text { FWHM } \\
\text { (5) }\end{array}$} & \multirow{2}{*}{$\begin{array}{c}T_{\text {eff }} / \log g / \xi_{t} \\
\text { (6) }\end{array}$} & \multicolumn{3}{|c|}{$[\mathrm{Fe} / \mathrm{H}]_{\text {spec }}$} \\
\hline & & & & & & $\begin{array}{l}\text { Fe I Lines } \\
\text { (7) }\end{array}$ & $\begin{array}{c}\text { Fe II Lines } \\
\text { (8) }\end{array}$ & $\begin{array}{l}\text { Adopted } \\
\text { (9) }\end{array}$ \\
\hline \multicolumn{9}{|c|}{ (a) 17 Non-Blazhko RRab-type stars } \\
\hline NR Lyr & CFHT & 0.29 & 37 & $0.163(02)$ & $6500 / 2.6 / 2.5$ & $-2.54 \pm 0.13(6)$ & $-2.53 \pm 0.17(5)$ & $-2.54 \pm 0.11$ \\
\hline FN Lyr & CFHT & 0.28 & 32 & $0.209(19)$ & $6300 / 2.44 / 2.5$ & $-2.00 \pm 0.13(10)$ & $-1.97 \pm 0.12(12)$ & $-1.98 \pm 0.09$ \\
\hline NQ Lyr & CFHT & 0.32 & 30 & $0.197(15)$ & $6000 / 1.80 / 2.5$ & $-1.88 \pm 0.19(11)$ & $-1.90 \pm 0.10(3)$ & $-1.89 \pm 0.10$ \\
\hline V350 Lyr & Keck & 0.53 & 34 & $0.293(11)$ & $6180 / 2.9 / 2.6$ & $-1.84 \pm 0.15(12)$ & $-1.81 \pm 0.14(10)$ & $-1.83 \pm 0.12$ \\
\hline V894 Cyg & CFHT & 0.39 & 28 & $0.193(08)$ & $6200 / 2.46 / 2.0$ & $-1.65 \pm 0.18(11)$ & $-1.66 \pm 0.16(9)$ & $-1.66 \pm 0.12$ \\
\hline AW Dra & CFHT & 0.30 & 38 & $0.202(05)$ & $6540 / 2.40 / 2.5$ & $-1.33 \pm 0.13(9)$ & $-1.33 \pm 0.12(6)$ & $-1.33 \pm 0.09$ \\
\hline KIC 7030715 & CFHT & 0.26 & 19 & $0.26(04)$ & $6500 / 2.64 / 2.5$ & $-1.32 \pm 0.07(6)$ & $-1.34 \pm 0.15(3)$ & $-1.33 \pm 0.08$ \\
\hline V1107 Cyg & Keck & 0.48 & 21 & $0.220(17)$ & $6300 / 2.8 / 3.0$ & $-1.23 \pm 0.42(33)$ & $-1.31 \pm 0.18(11)$ & $-1.29 \pm 0.23$ \\
\hline V368 Lyr & Keck & 0.43 & 20 & $0.183(02)$ & $6300 / 2.8 / 3.0$ & $-1.33 \pm 0.31(22)$ & $-1.25 \pm 0.24(10)$ & $-1.28 \pm 0.20$ \\
\hline KIC 9658012 & Keck & 0.84 & 37 & $0.413(16)$ & $6500 / 2.8 / 3.0$ & $-1.22 \pm 0.26(26)$ & $-1.31 \pm 0.12(11)$ & $-1.28 \pm 0.14$ \\
\hline KIC 9717032 & Keck & 0.23 & 19 & $0.262(06)$ & $6620 / 2.8 / 3.5$ & $-1.27 \pm 0.17(14)$ & $-1.26 \pm 0.17(13)$ & $-1.27 \pm 0.15$ \\
\hline V715 Cyg & Keck & 0.51 & 21 & $0.241(13)$ & $6400 / 3.0 / 3.0$ & $-1.13 \pm 0.12(25)$ & $-1.14 \pm 0.14(12)$ & $-1.13 \pm 0.09$ \\
\hline V2470 Cyg & CFHT & 0.32 & 26 & $0.262(11)$ & $6400 / 2.44 / 2.6$ & $-0.59 \pm 0.14(45)$ & $-0.59 \pm 0.21(14)$ & $-0.59 \pm 0.13$ \\
\hline V782 Cyg & Keck & 0.42 & 34 & $0.290(12)$ & $6525 / 3.2 / 2.9$ & $-0.40 \pm 0.15(102)$ & $-0.43 \pm 0.14$ & $-0.42 \pm 0.10$ \\
\hline KIC 6100702 & CFHT & 0.26 & 23 & $0.281(10)$ & $6500 / 2.50 / 2.5$ & $-0.19 \pm 0.14$ & $-0.13 \pm 0.12(6)$ & $-0.16 \pm 0.09$ \\
\hline V839 Cyg & Keck & 0.12 & 70 & $0.375(03)$ & $7200 / 3.1 / 4.0$ & $+0.07 \pm 0.18(137)$ & $-0.09 \pm 0.14(16)$ & $-0.05 \pm 0.14$ \\
\hline V784 Cyg & Keck & 0.47 & 27 & $0.323(12)$ & $6400 / 3.3 / 4.3$ & $-0.05 \pm 0.15(107)$ & $-0.06 \pm 0.13(10)$ & $-0.05 \pm 0.10$ \\
\hline \multicolumn{9}{|c|}{ (b) 12 Blazhko RRab-type stars (excluding RR Lyrae) } \\
\hline V2178 Cyg & Keck & 0.43 & 41 & $0.211(09)$ & $6450 / 2.8 / 3.0$ & $-1.63 \pm 0.24(17)$ & $-1.67 \pm 0.09(11)$ & $-1.66 \pm 0.13$ \\
\hline V450 Lyr & Keck & 0.57 & 21 & $0.236(17)$ & $6450 / 3.0 / 3.0$ & $-1.52 \pm 0.13(13)$ & $-1.50 \pm 0.21(9)$ & $-1.51 \pm 0.12$ \\
\hline V353 Lyr & Keck & 0.32 & 20 & $0.205(17)$ & $6300 / 2.8 / 3.0$ & $-1.48 \pm 0.20(15)$ & $-1.50 \pm 0.35(4)$ & $-1.50 \pm 0.20$ \\
\hline V360 Lyr & Keck & 0.52 & 29 & $0.378(20)$ & $6400 / 2.8 / 10$ & $-1.46 \pm 0.37(72)$ & $-1.54 \pm 0.44(26)$ & $-1.50 \pm 0.29$ \\
\hline V354 Lyr & Keck & 0.26 & 30 & $0.298(14)$ & $6400 / 2.8 / 3.0$ & $-1.44 \pm 0.24(20)$ & $-1.44 \pm 0.20(13)$ & $-1.44 \pm 0.16$ \\
\hline V1104 Cyg & Keck & 0.42 & 33 & $0.174(03)$ & $6300 / 2.8 / 3.0$ & $-1.25 \pm 0.25(51)$ & $-1.21 \pm 0.15(17)$ & $-1.23 \pm 0.15$ \\
\hline V808 Cyg & Keck & 0.70 & 19 & $0.473(16)$ & $6300 / 2.8 / 3.0$ & $-1.20 \pm 0.27(15)$ & $-1.18 \pm 0.25(7)$ & $-1.19 \pm 0.18$ \\
\hline V783 Cyg & CFHT & 0.58 & 16 & $0.23(01)$ & $6400 / 2.1 / 2.6$ & $-1.19 \pm 0.18(13)$ & $-1.13 \pm 0.14(2)$ & $-1.16 \pm 0.11$ \\
\hline V366 Lyr & Keck & 0.69 & 21 & $0.332(24)$ & $6430 / 3.0 / 3.0$ & $-1.13 \pm 0.13(16)$ & $-1.19 \pm 0.10(10)$ & $-1.16 \pm 0.09$ \\
\hline V355 Lyr & CFHT & 0.19 & 17 & $0.247(16)$ & $6900 / 3.28 / 2.5$ & $-1.13 \pm 0.14(10)$ & $-1.17 \pm 0.30(1)$ & $-1.14 \pm 0.17$ \\
\hline KIC 11125706 & CFHT & 0.33 & 69 & $0.232(04)$ & $6200 / 2.35 / 2.25$ & $-1.09 \pm 0.13$ & $-1.09 \pm 0.10(17)$ & $-1.09 \pm 0.08$ \\
\hline V838 Cyg & CFHT & 0.36 & 13 & $0.236(28)$ & $6850 / 2.12 / 2.4$ & $-1.02 \pm 0.19$ & $-1.00 \pm 0.10(3)$ & $-1.01 \pm 0.10$ \\
\hline \multicolumn{9}{|c|}{ (c) Five RRc-type stars (including the RRc/HADS? star KIC 3868420) } \\
\hline KIC 9453114 & CFHT & 0.28 & 29 & $0.278(27)$ & $6500 / 2.5 / 2.5$ & & $-2.13 \pm 0.12(4)$ & $-2.13 \pm 0.12$ \\
\hline KIC 4064484 & Keck & 0.59 & 33 & $0.288(11)$ & $6500 / 2.8 / 3.0$ & $-1.65 \pm 0.22(9)$ & $-1.54 \pm 0.12(6)$ & $-1.58 \pm 0.13$ \\
\hline KIC 3868420 & CFHT & 0.47 & 96 & $0.359(09)$ & $7480 / 3.9 / 2.0$ & $-0.35 \pm 0.14(13)$ & $-0.29 \pm 0.08(6)$ & $-0.31 \pm 0.08$ \\
\hline KIC 8832417 & CFHT & 0.29 & 30 & $0.349(16)$ & $7000 / 3.30 / 2.5$ & $-0.22 \pm 0.19(27)$ & $-0.29 \pm 0.09(7)$ & $-0.27 \pm 0.11$ \\
\hline KIC 5520878 & Keck & 0.39 & 40 & $0.319(02)$ & $7250 / 3.9 / 2.4$ & $-0.18 \pm 0.18(76)$ & $-0.19 \pm 0.12(18)$ & $-0.18 \pm 0.10$ \\
\hline \multicolumn{9}{|c|}{ (d) Nine bright RR Lyrae metal abundance standards (including RR Lyrae) } \\
\hline X Ari & Keck & 0.31 & 223 & $0.188(05)$ & $6075 / 2.4 / 2.0$ & $-2.75 \pm 0.08(9)$ & $-2.74 \pm 0.17(11)$ & $-2.74 \pm 0.09$ \\
\hline VY Ser & Keck & 0.45 & 120 & $0.232(03)$ & $6170 / 2.8 / 2.5$ & $-1.73 \pm 0.12(29)$ & $-1.69 \pm 0.08(16)$ & $-1.71 \pm 0.07$ \\
\hline ST Boo & Keck & 0.36 & 135 & $0.198(02)$ & $6313 / 2.6 / 1.8$ & $-1.66 \pm 0.12(39)$ & $-1.60 \pm 0.04(15)$ & $-1.62 \pm 0.06$ \\
\hline UU Cet & Keck & 0.38 & 66 & $0.226(03)$ & $6150 / 2.6 / 1.6$ & $-1.34 \pm 0.14(68)$ & $-1.32 \pm 0.07(19)$ & $-1.33 \pm 0.08$ \\
\hline RR Lyr & Keck & 0.54 & 252 & $0.276(06)$ & $6400 / 2.8 / 3.0$ & $-1.27 \pm 0.22$ & $-1.27 \pm 0.09$ & $-1.27 \pm 0.12$ \\
\hline VX Her & Keck & 0.32 & 97 & $0.202(02)$ & $6690 / 2.9 / 2.2$ & $-1.22 \pm 0.17(51)$ & $-1.23 \pm 0.17(17)$ & $-1.23 \pm 0.12$ \\
\hline RR Cet & Keck & 0.37 & 184 & $0.212(03)$ & $6420 / 2.9 / 1.9$ & $-1.17 \pm 0.15(65)$ & $-1.19 \pm 0.08(17)$ & $-1.18 \pm 0.09$ \\
\hline V445 Oph & Keck & 0.44 & 106 & $0.315(05)$ & $6200 / 2.8 / 1.8$ & $+0.15 \pm 0.14(218)$ & $+0.11 \pm 0.13(16)$ & $+0.13 \pm 0.10$ \\
\hline SW And & Keck & 0.56 & 151 & $0.367(06)$ & $6660 / 3.6 / 2.2$ & $+0.19 \pm 0.14$ & $+0.21 \pm 0.09$ & $+0.20 \pm 0.08$ \\
\hline
\end{tabular}

Notes. The columns contain: (1) star name; (2) source of the spectra, either "Keck I 10 m + HIRES" or "CFHT 3.6 m + ESPaDOnS"; (3) pulsation phase at mid-exposure; (4) signal-to-noise ratio per pixel for the co-added spectra, measured at $~ 570 \mathrm{~nm}$ and calculated using the VWA "rainbow" widget; (5) average FWHM value $(\AA)$ for the three Fe II lines at 4508,4515 , and $4520 \AA$, derived from the spectrum (either single or co-added exposures) analyzed by VWA-the uncertainty (given in parentheses) is approximated by the standard deviation of the mean FWHM for the three lines; (6) the assumed values for the model effective temperature $T_{\text {eff }}(\mathrm{K})$, surface gravity $\log g\left(\mathrm{~cm} \mathrm{~s}^{-2}\right)$, and microturbulent velocity $\xi_{t}\left(\mathrm{~km} \mathrm{~s}^{-1}\right)$-in general the $T_{\text {eff }}$ have uncertainties $\sim \pm 150 \mathrm{~K}$, the $\log g$ are \pm 0.10 to 0.20 , and the $\xi_{t}$ are \pm 0.3 to $0.5 \mathrm{~km} \mathrm{~s}^{-1}$; (7) [Fe/H] abundance based on the Fe I lines (number of lines given in parentheses); (8) [Fe/H] abundance based on the Fe II lines (number of lines given in parentheses); (9) adopted VWA spectroscopic metal abundance, equal to the weighted (in inverse proportion to uncertainty) average of the abundances in Columns 7 and 8. 
Table 8

Spectroscopic Iron-to-Hydrogen Abundances (MOOG)

\begin{tabular}{|c|c|c|c|c|c|}
\hline $\begin{array}{l}\text { Star } \\
(1)\end{array}$ & $\begin{array}{c}T_{\text {eff }} / \log g / \xi_{t} /[\mathrm{Fe} / \mathrm{H}] \\
(2)\end{array}$ & $\begin{array}{c}\log \epsilon(\mathrm{Fe} I) \\
\text { (3) }\end{array}$ & $\begin{array}{c}\log \epsilon(\mathrm{Fe} \mathrm{II}) \\
(4)\end{array}$ & $\begin{array}{c}\log \epsilon(\mathrm{Fe}) \\
\quad(5)\end{array}$ & $\begin{array}{c}{[\mathrm{Fe} / \mathrm{H}]_{\text {spec }}} \\
\text { (6) }\end{array}$ \\
\hline X Ari & $6000 / 3.0 / 2.0 /-2.5$ & $4.73 \pm 0.12(105)$ & $5.19 \pm 0.18(24)$ & $4.91 \pm 0.07$ & $-2.59 \pm 0.12$ \\
\hline VY Ser & $6250 / 3.0 / 2.0 /-1.5$ & $5.83 \pm 0.12(147)$ & $6.01 \pm 0.12(26)$ & $5.92 \pm 0.06$ & $-1.58 \pm 0.11$ \\
\hline ST Boo & $6250 / 3.0 / 2.0 /-1.5$ & $5.78 \pm 0.12(177)$ & $6.09 \pm 0.12(29)$ & $5.94 \pm 0.06$ & $-1.57 \pm 0.11$ \\
\hline RR Lyr & $6000 / 3.0 / 2.0 /-1.5$ & $5.96 \pm 0.14(145)$ & $6.25 \pm 0.09(19)$ & $6.14 \pm 0.05$ & $-1.36 \pm 0.10$ \\
\hline VX Her & $6750 / 3.0 / 2.0 /-1.5$ & $6.24 \pm 0.12(169)$ & $6.35 \pm 0.13(24)$ & $6.29 \pm 0.06$ & $-1.21 \pm 0.11$ \\
\hline SW And & $6250 / 3.0 / 2.0 /+0.0$ & $7.35 \pm 0.15(177)$ & $7.42 \pm 0.15(11)$ & $7.39 \pm 0.08$ & $-0.12 \pm 0.13$ \\
\hline V782 Cyg & $6250 / 3.0 / 2.0 /+0.0$ & $7.41 \pm 0.30(162)$ & $7.45 \pm 0.28(11)$ & $7.43 \pm 0.14$ & $-0.07 \pm 0.19$ \\
\hline V445 Oph & $6250 / 3.0 / 2.0 /+0.0$ & $7.45 \pm 0.11(188)$ & $7.61 \pm 0.13(11)$ & $7.52 \pm 0.06$ & $+0.02 \pm 0.11$ \\
\hline V784 Cyg & $6250 / 3.0 / 2.0 /+0.0$ & $7.86 \pm 0.35(142)$ & $7.92 \pm 0.34(7)$ & $7.89 \pm 0.17$ & $+0.39 \pm 0.22$ \\
\hline
\end{tabular}

Notes. The columns contain: (1) the star name; (2) the assumed values for the (WebMARCS) model effective temperature, $T_{\text {eff }}(\mathrm{K})$, surface gravity, $\log g\left(\mathrm{~cm} \mathrm{~s}^{-2}\right)$, microturbulent velocity, $\xi_{t}\left(\mathrm{~km} \mathrm{~s}^{-1}\right)$, and metal abundance, $[\mathrm{Fe} / \mathrm{H}] ;(3)$ the $[\mathrm{Fe} \mathrm{I} / \mathrm{H}]$ abundance $($ based on the number of Fe I lines given in parentheses); (4) the [Fe II/H] abundance (based on the number of Fe II lines given in parentheses); (5) the weighted average of the $[\mathrm{Fe} \mathrm{I} / \mathrm{H}]$ and $[\mathrm{Fe} \mathrm{II} / \mathrm{H}]$ abundances; (6) the adopted MOOG spectroscopic iron abundance.

\& Piskunov (1996). ${ }^{24}$ SME compares observed spectra with synthetic spectra, where the latter are computed by solving the radiative transfer equations. Parameter estimates are calculated by minimizing (using Levenberg-Marquart) a $\chi^{2}$ statistic that measures the lack of agreement between the observed and fitted intensities. Unlike VWA and MOOG, which are based on comparing calculated and measured EWs, SME matches sections of spectra. SME does not actually calculate Fe I and Fe II abundances, but rather, the abundances assumed for the Sun are scaled and used to calculate a mean metal abundance, $[\mathrm{M} / \mathrm{H}]$, which we assume to be comparable to $[\mathrm{Fe} / \mathrm{H}]$.

The spectra that were analyzed with SME were the co-added normalized Keck and CFHT spectra and the Kurucz-Hinckle solar spectrum. The derived photospheric quantities were $T_{\text {eff }}$, $\log g$ (cgs units), $\xi_{t}, v_{\mathrm{mac}}, v \sin i$, and $[\mathrm{M} / \mathrm{H}]$. For most of the SME analyses the synthetic spectra were calculated for the $\lambda$-interval $500-520 \mathrm{~nm}$, for which VALD returned atomic parameters for 476 lines. This interval is free of Balmer lines, around which the continua were usually poorly defined. The interval 480-520 nm was tried, but spurious results were found, presumably owing to the presence of $\mathrm{H} \gamma$. For several stars the interval $440-460 \mathrm{~nm}$ was also analyzed, using 952 lines identified by VALD, and the results were averaged with those from $500-520 \mathrm{~nm}$.

Our estimates of the atmospheric parameters were obtained iteratively by varying the initial values supplied to SME until the "best" overall estimates were found. The usual reduction procedure was to take as starting values the parameter set calculated by VWA (see Table 7) and then to solve simultaneously for two parameters at a time. First $\xi_{t}$ and $v_{\text {mac }}$ were calculated, then $v \sin i$ and $R(=\lambda / \Delta \lambda)$, and then $T_{\text {eff }}$ and $\log g$. After each run the prior input values were replaced with the revised values. Last, with the newer set of SME parameters, we solved for $[\mathrm{M} / \mathrm{H}]$.

Table 9 contains the final results of the SME analyses, sorted in order of increasing metal abundance. A total of 47 stars were analyzed. The co-added spectrum of KIC 9973633 was not measured owing to its very low $\mathrm{S} / \mathrm{N}$.

\subsubsection{Comparison of Atmospheric Parameters}

In Table 10 the VWA, MOOG, and SME iron abundances are compared. Also included for comparison with our values are the $[\mathrm{Fe} / \mathrm{H}]$ values derived by L94, Suntzeff et al. (1994), C95,

\footnotetext{
24 SME was written in IDL and is freely available at http://www.stsci.edu/ valenti/sme/.
}

Lambert et al. (1996, hereafter L96), Feast et al. (2008), and Wallerstein \& Huang (2010). Because C95 assumed $\log \epsilon(\mathrm{Fe})=$ 7.56, whereas we adopted the value $\log \epsilon(\mathrm{Fe})=7.50$, the $\mathrm{C} 95$ metallicity values (from their Table 12) have been increased by 0.06 dex. L96 report $\log \epsilon$ (Fe II) values, to which we have added 7.50 to give the abundances in Column 5. The L94 metallicities are based on measurements of the Ca II K-line.

$\mathrm{X}$ Ari, with $[\mathrm{Fe} / \mathrm{H}] \sim-2.65 \mathrm{dex}$, is probably more metal deficient than NR Lyr, the most metal-poor RR Lyr star in the Kepler field, which we estimated to have $[\mathrm{Fe} / \mathrm{H}]=$ $-2.54 \pm 0.11$ dex (Table 7). Recently Haschke et al. (2012) derived a spectroscopic $[\mathrm{Fe} / \mathrm{H}]=-2.61$ dex for $\mathrm{X}$ Ari, a value consistent with our estimate and with the value derived by Wallerstein \& Huang (2010). It is unclear whether the metal-rich end is defined by the standard star V445 Oph or by SW And. Our VWA and SME analyses suggest that the $[\mathrm{Fe} / \mathrm{H}]$ values for both stars are greater than that of the Sun, while the MOOG analyses suggest that SW And may be more metal-poor and V445 Oph more metal rich than the Sun. Most previous analyses lean toward V445 Oph being the more metal rich, as does our photometric $[\mathrm{Fe} / \mathrm{H}]$ (see Figures 4 and 12). In any case, both have metallicities similar to that of the most metal-rich star in the Kepler field, V784 Cyg.

For VY Ser a metal abundance $[\mathrm{Fe} / \mathrm{H}]=-1.77 \pm 0.10 \mathrm{dex}$ was derived by Carney \& Jones (1983). The good agreement between this value and our VWA, MOOG, and SME estimates is undoubtedly due to their spectra and ours having been taken at similar phases $(0.61$ versus our 0.48$)$. Furthermore, their $\log g=2.3 \pm 0.3$ and $\xi_{t}=4.2 \pm 0.5 \mathrm{~km} \mathrm{~s}^{-1}$ agree with our SME values of 2.1 (cgs units) and $4.4 \mathrm{~km} \mathrm{~s}^{-1}$; and their $T_{\text {eff }}=$ $6000 \pm 150 \mathrm{~K}$ is in accord with our SME estimate of $6167 \mathrm{~K}$.

In Figure 8 the SME estimates of the atmospheric parameters $T_{\text {eff }}, \log g, \xi_{t}$, and $[\mathrm{M} / \mathrm{H}]$ are compared (solid squares) with the values derived using VWA. While the results are similar, the temperatures from SME tend to be smaller than the VWA values (upper left panel), the mean difference being $\sim 100 \mathrm{~K}$, with an rms scatter $\sim 100 \mathrm{~K}$. The surface gravities from SME are also smaller on average than the VWA values (lower left panel)—mean $\Delta \log g \sim-0.5$ with an rms scatter of 0.3 . The trend seen in $\xi_{t}$ (upper right panel) implies that the SMEderived values are systematically larger than the VWA values in most cases, where the difference increases as $\xi_{t}$ increases (not shown in the diagram is the extreme outlier V360 Lyr, where $\Delta \xi_{t}=-4.7 \mathrm{~km} \mathrm{~s}^{-1}$ is due primarily to the large $\xi_{t}$ $\left(=10 \mathrm{~km} \mathrm{~s}^{-1}\right)$ adopted for the VWA analysis). Despite these 
Table 9

Physical Characteristics Derived Using SME

\begin{tabular}{|c|c|c|c|c|c|c|}
\hline $\begin{array}{l}\text { Star } \\
\text { (1) }\end{array}$ & $\begin{array}{l}T_{\text {eff }} \\
(2)\end{array}$ & $\begin{array}{c}\log g \\
(3)\end{array}$ & $\begin{array}{l}\xi_{t} \\
(4)\end{array}$ & $\begin{array}{c}v_{\mathrm{mac}} \\
(5)\end{array}$ & $\begin{array}{c}v \sin i \\
(6)\end{array}$ & $\begin{array}{c}{[\mathrm{M} / \mathrm{H}]} \\
(7)\end{array}$ \\
\hline X Ari $\dagger$ & 6200 & 1.85 & 4.8 & 9.5 & 2.7 & -2.71 \\
\hline NR Lyr $\dagger$ & 6363 & 2.56 & 4.4 & 7.9 & 4.8 & -2.51 \\
\hline KIC 9453414 & 6204 & 2.04 & 6.4 & 29.3 & 4.3 & -2.15 \\
\hline V1510 Cyg & 5706 & 1.76 & 4.9 & 10.8 & 4.9 & -2.00 \\
\hline FN Lyr & 6203 & 2.21 & 4.7 & 8.0 & 4.6 & -1.91 \\
\hline NQ Lyr & 5980 & 1.56 & 4.4 & 8.4 & 4.5 & -1.87 \\
\hline V350 Lyr & 6131 & 2.29 & 5.1 & 16.7 & 3.0 & -1.82 \\
\hline V445 Lyr & 6227 & 1.70 & 5.3 & 12.4 & 5.4 & -1.78 \\
\hline VY Ser & 6167 & 2.10 & 4.4 & 11.3 & 3.2 & -1.71 \\
\hline V2178 Cyg & 6301 & 2.35 & 4.2 & 9.2 & 4.3 & -1.66 \\
\hline V894 Cyg & 6220 & 1.92 & 3.8 & 7.2 & 4.4 & -1.65 \\
\hline ST Boo $\dagger$ & 6327 & 1.91 & 3.8 & 10.5 & 2.1 & -1.61 \\
\hline V353 Lyr & 6093 & 1.84 & 4.8 & 4.8 & 4.1 & -1.57 \\
\hline V450 Lyr & 6280 & 2.34 & 3.6 & 12.7 & 5.0 & -1.51 \\
\hline KIC 4064484 & 6473 & 2.39 & 4.0 & 18.4 & 4.9 & -1.51 \\
\hline V360 Lyr & 6202 & 2.42 & 5.3 & 18.9 & 5.0 & -1.48 \\
\hline V346 Lyr & 6108 & 2.34 & 4.2 & 9.6 & 5.0 & -1.48 \\
\hline V354 Lyr & 6391 & 2.05 & 4.6 & 15.0 & 4.2 & -1.43 \\
\hline V1107 Cyg & 6087 & 2.23 & 5.1 & 11.4 & 3.0 & -1.40 \\
\hline VX Her & 6533 & 2.46 & 3.8 & 9.0 & 2.2 & -1.38 \\
\hline KIC 7030715 & 6221 & 1.99 & 4.2 & 13.5 & 10.0 & -1.37 \\
\hline AW Dra & 6461 & 2.02 & 4.4 & 5.9 & 3.6 & -1.34 \\
\hline UU Cet & 6269 & 2.31 & 4.2 & 9.1 & 2.4 & -1.33 \\
\hline RR Lyr $†$ & 6445 & 3.13 & 5.1 & 11.7 & 5.6 & -1.33 \\
\hline V368 Lyr & 6231 & 1.93 & 3.3 & 9.5 & 3.0 & -1.29 \\
\hline KIC 9658012 & 6314 & 2.15 & 4.3 & 15.0 & 12.9 & -1.27 \\
\hline KIC 9717032 & 6461 & 2.14 & 5.0 & 14.8 & 9.8 & -1.24 \\
\hline RR Cet & 6459 & 2.40 & 4.0 & 8.8 & 3.8 & -1.22 \\
\hline V1104 Cyg & 6352 & 2.08 & 2.0 & 0.4 & 2.0 & -1.19 \\
\hline V355 Lyr & 6869 & 2.29 & 5.8 & 18.3 & 5.0 & -1.15 \\
\hline V715 Cyg & 6265 & 2.19 & 3.7 & 11.1 & 4.6 & -1.14 \\
\hline V808 Cyg & 6169 & 2.19 & 6.4 & 25.2 & 4.3 & -1.14 \\
\hline V366 Lyr & 6263 & 2.26 & 5.1 & 19.8 & 5.3 & -1.13 \\
\hline V783 Cyg & 6122 & 1.59 & 3.9 & 14.3 & 5.0 & -1.10 \\
\hline KIC 11125706 & 6145 & 1.67 & 2.3 & 14.7 & 6.6 & -1.09 \\
\hline V838 Cyg & 6600 & 1.81 & 4.3 & 14.0 & 5.0 & -1.01 \\
\hline V2470 Cyg & 6220 & 2.13 & 2.6 & 9.7 & 6.5 & -0.59 \\
\hline V782 Cyg & 6446 & 3.17 & 3.7 & 12.0 & 4.6 & -0.42 \\
\hline V839 Cyg † & 6982 & 2.46 & 3.5 & 18.4 & 5.4 & -0.31 \\
\hline KIC $3868420 \dagger$ & 7612 & 3.71 & 2.3 & 23.0 & 5.7 & -0.28 \\
\hline KIC 8832417 & 6999 & 3.30 & 3.5 & 15.3 & 4.8 & -0.25 \\
\hline KIC 5520878 & 7266 & 3.66 & 3.0 & 15.2 & 5.1 & -0.18 \\
\hline KIC 6100702 & 6471 & 2.83 & 3.1 & 10.2 & 4.7 & -0.18 \\
\hline Sun & 5770 & 4.44 & 0.8 & 8.7 & 2.1 & +0.00 \\
\hline V784 Cyg & 6305 & 2.84 & 3.6 & 13.4 & 5.7 & +0.00 \\
\hline V445 Oph & 6496 & 3.15 & 2.9 & 12.0 & 2.5 & +0.14 \\
\hline SW And & 6996 & 3.70 & 4.2 & 16.2 & 2.9 & +0.19 \\
\hline
\end{tabular}

Notes. Most of the entries in this table are based on analysis of 476 lines in the wavelength interval $500-520 \mathrm{~nm}$. The stars with a $†$ following the name include additional measurements made using 952 lines in the interval 440-460 nm. The names of the nine Keck standard stars (and the Sun) are printed in "boldface." The V1104 Cyg results are based on analysis of the Keck spectrum (the CFHT spectra for this star were too noisy to measure).

differences the lower right panel shows that there is reasonably good agreement of the $[\mathrm{M} / \mathrm{H}]$ abundances derived with SME and the $[\mathrm{Fe} / \mathrm{H}]$ values derived using VWA (and MOOG) - the outliers at the bottom of the panel are V1107 Cyg, V839 Cyg, and VX Her (a standard star). For the VWA analyses, the $v_{\text {mac }}$ values were generally set to $2-3 \mathrm{~km} \mathrm{~s}^{-1}$ and $v \sin i$ values of $2 \mathrm{~km} \mathrm{~s}^{-1}$ and $7-10 \mathrm{~km} \mathrm{~s}^{-1}$ were adopted for the Keck and CFHT spectra, respectively. These values differ considerably from the $v_{\text {mac }}$ values derived by SME, which have a mean of $13.0 \mathrm{~km} \mathrm{~s}^{-1}$ (standard deviation $\sigma=5.4 \mathrm{~km} \mathrm{~s}^{-1}, N=43$ stars), and from the $v \sin i$ values (see bottom panel of Figure 9) derived by SME, which have a mean of $4.3 \mathrm{~km} \mathrm{~s}^{-1}\left(\sigma=1.2 \mathrm{~km} \mathrm{~s}^{-1}, N=40\right.$, excluding the three stars with $\left.v \sin i>9 \mathrm{~km} \mathrm{~s}^{-1}\right)$.

Also plotted in Figure 8 (as crosses) are the differences between our SME-derived parameters for the nine Keck RR Lyrae standard stars and the corresponding values derived by C95. Since the pulsation phases for our Keck spectra differ from those of the Palomar $1.5 \mathrm{~m}$ spectra analyzed by C95 the effective temperatures are expected to differ. On the other hand, because the RRab stars lie on the red side of the instability strip and both studies used spectra taken away from maximum light the $T_{\text {eff }}$ should be similar; in fact, $\Delta T_{\text {eff }}(\mathrm{SME}-\mathrm{C} 95$ ) (upper left panel) does not exceed $700 \mathrm{~K}$ and is typically $\sim 200 \mathrm{~K}$. The largest differences occur for VX Her $(558 \mathrm{~K})$ and for SW And $(659 \mathrm{~K})$. The difference for VX Her is readily explained by the different $\phi_{\text {puls }}$ values: the average pulsation phase for the five C95 spectra is $\phi_{\text {puls }}=0.61$ and therefore they measured $T_{\text {eff }}=$ $5978 \mathrm{~K}$; on the other hand the Keck spectrum was taken at $\phi_{\text {puls }}=0.32$ and the average $T_{\text {eff }}=6660 \pm 70 \mathrm{~K}$ from the VWA, MOOG, and SME analyses is hotter. The large temperature difference for SW And is problematic. The average $\phi_{\text {puls }}$ for the three C95 spectra is 0.82 , which is to be compared with $\phi_{\text {puls }}=0.56$ for our Keck spectrum. According to For et al. (2011, their Figures 8-10) both phases are near minimum temperature and therefore $T_{\text {eff }} \sim 6200 \mathrm{~K}$ is expected in both cases. The $T_{\text {eff }}=6337 \mathrm{~K}$ derived by C95 is consistent with this prediction, as is the $T_{\text {eff }}$ from our MOOG analysis; however, both the VWA and SME analyses suggest a higher temperature, and as a consequence a slightly higher $[\mathrm{Fe} / \mathrm{H}]$ value.

The comparison of surface gravities (Figure 8, lower left panel) is intriguing, with the differences for the nine standard stars lying along the line $\Delta \log g=0.89 \log g(\mathrm{SME})-2.46$, i.e., the gravities derived by SME tend to be smaller than the C95 values when $\log g$ is less than 2.76 and larger when $\log g$ is greater than 2.76. A linear trend is also seen when our VWA $\xi_{t}$ estimates for the standard stars (crosses in the upper right panel) are compared with the C95 microturbulent parameter, where the former values are offset by $\sim 2 \mathrm{~km} \mathrm{~s}^{-1}$. Despite these atmospheric parameter differences, the metallicities derived here and by C95 are all within $\sim 0.2$ dex of each other (bottom right panel), with a difference $<0.1$ dex in most cases.

\subsubsection{Correlations of Atmospheric Parameters}

Figure 9 shows the SME-derived atmospheric parameters $v_{\text {mac }}, \xi_{t}$, and $v \sin i$, plotted as a function of $T_{\text {eff }}$. Each panel includes the values derived for the program RR Lyr stars, for the Sun, for the candidate HADS star KIC 3868420, and for the bright Keck RR Lyrae standard stars. As expected, the RRc stars, KIC 5520878 and KIC 8832417 (both of which are metal-rich) are the hottest among the RR Lyr stars. The candidate HADS star KIC 3868420 (also metal-rich) is even hotter. Differences between KIC 3868420 and the bona fide hot RRc stars appear to be comparatively small. While this observation lends support to the possibility that it may be an unusual metal-rich shortperiod RRc star, possibly an RRe (second overtone?) pulsator (Walker \& Nemec 1996; Kovács 1998), identification as a hot HADS star of 1.6 to 2 solar masses seems more likely. Balona $\&$ Nemec (2012) found that $\delta$ Sct stars hotter than $\log T_{\text {eff }}=$ 3.88 are quite common and suggested that KIC 3868420, based on its photometric and kinematic properties, is not an SX Phe star (i.e., a halo population $\delta$ Sct star such as are found 

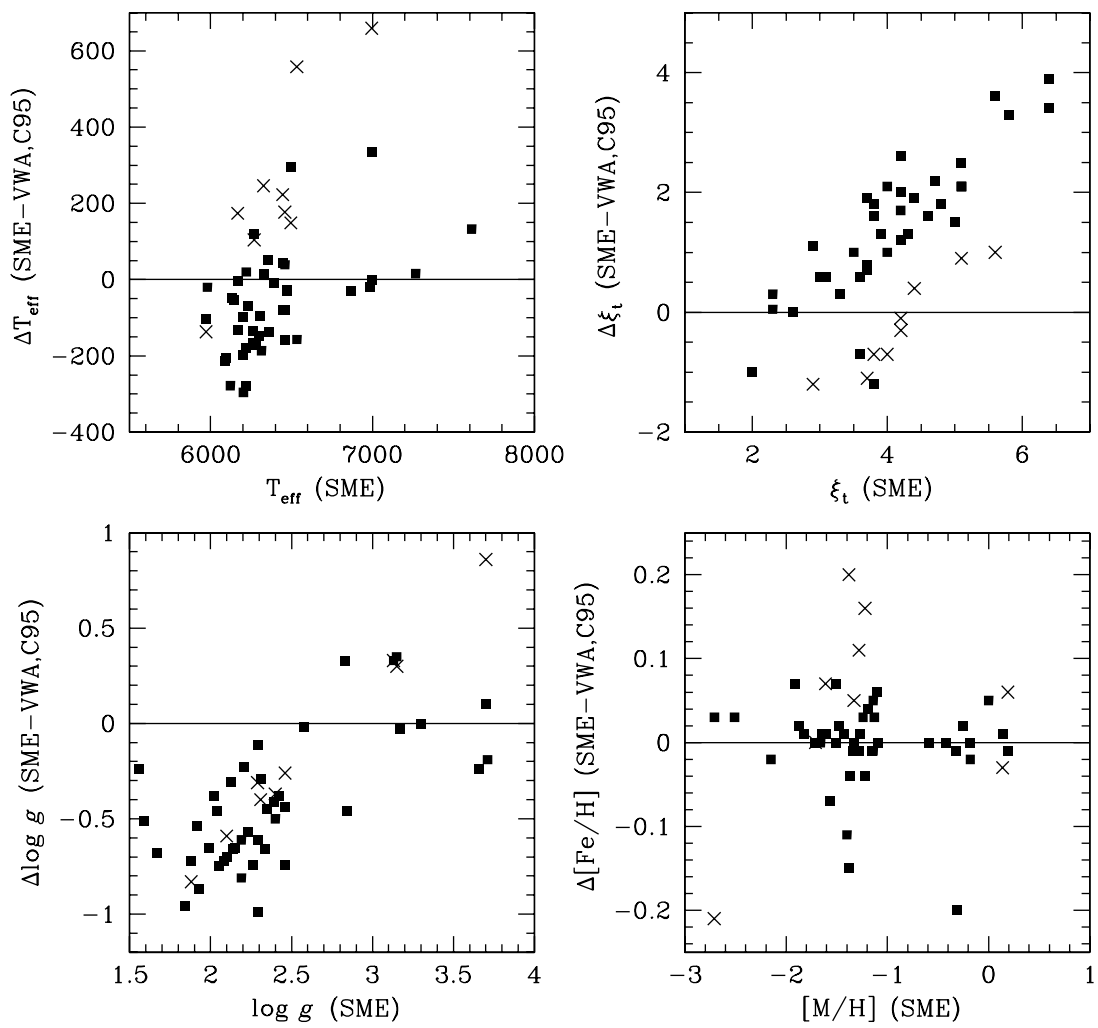

Figure 8. Comparison of physical parameters derived using SME with those derived using VWA (or assumed for the VWA analyses). Also shown (as crosses) are the parameter differences for the nine Keck standards, as derived using SME compared with the values derived independently by C95.

Table 10

Spectroscopic Iron-to-Hydrogen Abundances for the Keck Standard Stars

\begin{tabular}{|c|c|c|c|c|c|c|c|c|c|}
\hline \multirow{2}{*}{$\begin{array}{l}\text { Star } \\
\text { (1) }\end{array}$} & \multicolumn{6}{|c|}{$[\mathrm{Fe} / \mathrm{H}]$ (Previous Papers) } & \multicolumn{3}{|c|}{$[\mathrm{Fe} / \mathrm{H}]$ (This Paper) } \\
\hline & $\begin{array}{l}\text { L94 } \\
(2)\end{array}$ & $\begin{array}{c}\text { SKK94 } \\
\text { (3) }\end{array}$ & $\begin{array}{l}\text { C95 } \\
(4)\end{array}$ & $\begin{array}{l}\text { L96 } \\
(5)\end{array}$ & $\begin{array}{c}\text { Fer98 } \\
(6)\end{array}$ & $\begin{array}{c}\text { Wal10 } \\
\text { (7) }\end{array}$ & $\begin{array}{c}\text { VWA } \\
(8)\end{array}$ & $\begin{array}{l}\text { MOOG } \\
\text { (9) }\end{array}$ & $\begin{array}{l}\text { SME } \\
(10)\end{array}$ \\
\hline $\mathrm{X}$ Ari & -2.40 & -2.16 & -2.44 & -2.53 & -2.43 & -2.68 & $-2.74 \pm 0.09$ & $-2.59 \pm 0.12$ & $-2.71 \pm 0.10$ \\
\hline VY Ser & -1.82 & -1.83 & -1.65 & -1.75 & -1.79 & . & $-1.71 \pm 0.07$ & $-1.58 \pm 0.11$ & $-1.71 \pm 0.10$ \\
\hline ST Boo & -1.86 & $\ldots$ & -1.62 & $\ldots$ & -1.76 & -1.77 & $-1.62 \pm 0.06$ & $-1.57 \pm 0.11$ & $-1.61 \pm 0.10$ \\
\hline RR Cet & -1.52 & -1.55 & -1.32 & $\ldots$ & -1.45 & $\ldots$ & $-1.27 \pm 0.12$ & $\ldots$ & $-1.22 \pm 0.10$ \\
\hline UU Cet & $\ldots$ & -1.88 & -1.32 & $\ldots$ & -1.28 & $\ldots$ & $-1.33 \pm 0.08$ & $\ldots$ & $-1.33 \pm 0.10$ \\
\hline RR Lyr & -1.37 & -1.37 & -1.32 & -1.47 & -1.39 & $\ldots$ & $-1.27 \pm 0.12$ & $-1.36 \pm 0.10$ & $-1.33 \pm 0.10$ \\
\hline VX Her & $\ldots$ & -1.37 & -1.52 & $\ldots$ & -1.58 & -1.48 & $-1.23 \pm 0.12$ & $-1.21 \pm 0.11$ & $-1.38 \pm 0.10$ \\
\hline SW And & -0.38 & -0.41 & -0.00 & -0.23 & -0.24 & -0.16 & $+0.20 \pm 0.08$ & $-0.12 \pm 0.13$ & $+0.19 \pm 0.10$ \\
\hline V445 Oph & -0.23 & -0.30 & +0.23 & $\ldots$ & -0.19 & +0.24 & $+0.13 \pm 0.10$ & $+0.02 \pm 0.11$ & $+0.14 \pm 0.10$ \\
\hline
\end{tabular}

Notes. The columns contain: (1) the star name; (2) [Fe/H] from Table 9 of L94, where $[\mathrm{Fe} / \mathrm{H}]$ is based on the Ca II K-line; (3) [Fe/H] from Table 3 of Suntzeff et al. (1994), based on $\Delta S$ measurements; (4) $[\mathrm{Fe} / \mathrm{H}]$ from Table 12 of C95, made more metal rich by 0.06 since Clementini et al. (1995) assumed for the Sun log $\epsilon(\mathrm{Fe})=$ 7.56, whereas we are assuming 7.50; (5) [Fe/H] from Table 5 of Lambert et al. (1996) - the $\log \epsilon$ given, which are the Fe II values, have had the assumed solar Fe abundance (7.50) subtracted; (6) $[\mathrm{Fe} / \mathrm{H}]$ from Table 1 of Fernley et al. (1998)—these same values were adopted by Feast et al. (2008); (7) [Fe/H] from Table 2 of Wallerstein \& Huang (2010), based on analysis of Apache Point Observatory spectra; (8-10) spectroscopic [Fe/H] values derived here using VWA, MOOG, and SME.

among the blue stragglers in globular clusters). The metal-poor RRc star KIC 9453414 has the highest derived macroturbulent velocity of all the program stars, i.e., $v_{\mathrm{mac}}=29 \mathrm{~km} \mathrm{~s}^{-1}$. The three stars with the greatest projected rotation velocities are the non-Blazhko RRab stars KIC 9658012, KIC 9717032, and KIC K7030715-all the other stars have $v \sin i$ values smaller than $7 \mathrm{~km} \mathrm{~s}^{-1}$. The RRab star with the coolest $T_{\text {eff }}$ is V1510 Cyg, a non-Blazhko star for which we were unable to perform a successful VWA analysis of the CFHT spectra. V808 Cyg (a Blazhko star) also has a high $v_{\text {mac }}$ and V1104 Cyg (another Blazhko star) has the extremely low value of $v_{\text {mac }}=0.4 \mathrm{~km} \mathrm{~s}^{-1}$ (see top panel of Figure 9) For the standard stars (all with high
$\mathrm{S} / \mathrm{N}$ spectra) and the RRc stars, in Figure 9 three linear trends are apparent: the hottest stars tend to have higher $v_{\text {mac }}$, lower $\xi_{t}$, and higher $v \sin i$ velocities than the cooler stars. Equations of the fitted trend lines are given on the graphs. It is interesting to note that the $v_{\text {mac }}$ and $v \sin i$ trends are similar to those for the 23 bright solar-type stars studied by Bruntt et al. (2010a) and summarized in their Figure 11, although at a given $T_{\text {eff }}$ the RR Lyr stars appear to have higher $v_{\text {mac }}$ values than the solar-type stars.

In Figure 10 the atmospheric parameters derived with SME for the RR Lyr stars are compared with the same quantities (also derived using SME) for the 253 halo metal-poor red giants 


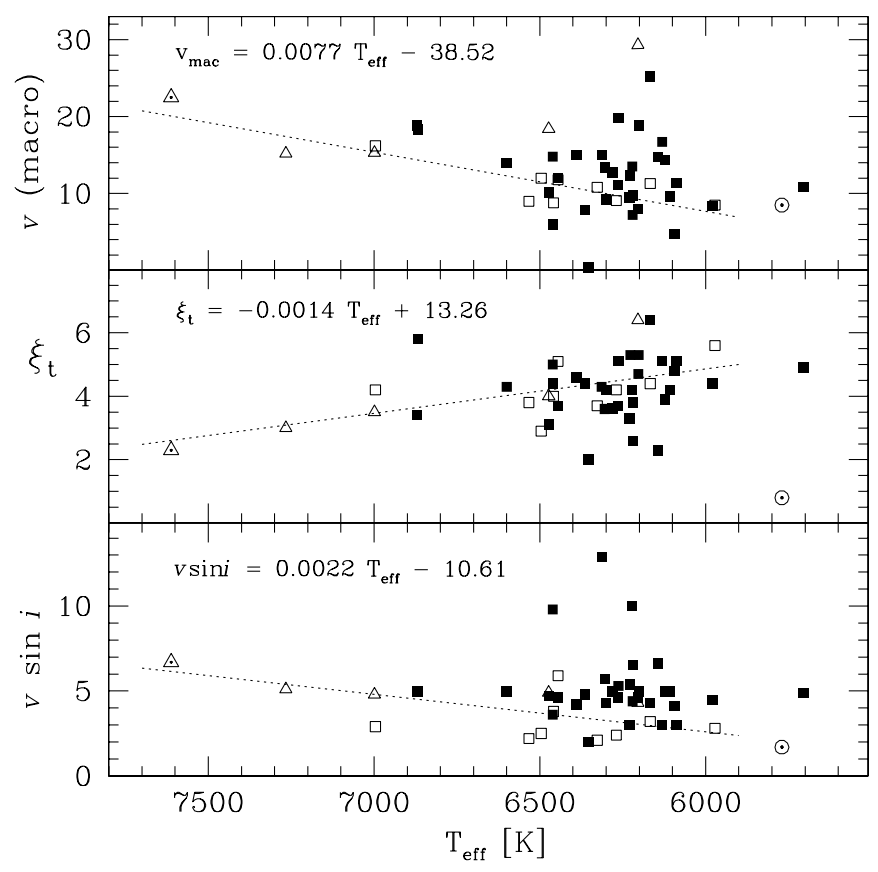

Figure 9. Macroturbulent velocity (top panel), microturbulent velocity (middle panel), and projected rotation velocity (bottom panel) for the program and standard stars, all derived with SME, and all with units of $\left(\mathrm{km} \mathrm{s}^{-1}\right)$. The equations of the fitted lines (based on the high-S/N standard star data only) are as indicated on the graphs. Included in the diagrams are the program star RRab stars (solid black squares), the RRc stars (open triangles), the bright Keck RRab standard stars (open squares), the Sun (circled dot) and the candidate HADS star KIC 3868420 (dotted triangle).

and red horizontal branch (RHB) stars investigated by Barklem et al. (2005) as part of the HERES survey. In all four panels KIC 3868420 (dotted triangle) appears to be similar to the two metal-rich RRc stars KIC 5520878 and KIC 8832417, suggesting again that it might be a short period RRc star and not a HADS star. The $\xi_{t}$ versus $\log g$ diagram (upper left panel) shows that the RR Lyrae stars have systematically higher microturbulent velocities than the HERES metal-poor red giants and RHB stars with the same $\log g$. The $\log g$ versus $T_{\text {eff }}$ diagram (upper right panel), where the $\log g$ scale is reversed, closely resembles an H-R diagram. At a given surface gravity the RR Lyrae stars are hotter than the RHB stars, which in turn are hotter than the metal-poor red giants. As before the hottest stars are the RRc stars (and KIC 3868420), and the two metal-rich RRc stars are seen to have higher surface gravities and temperatures than the two metal-poor RRc stars. The $v_{\text {mac }}$ versus projected rotational velocity diagram (lower left panel) identifies those stars with the most extreme values of $v_{\text {mac }}$ and $v \sin i$ (see earlier discussion). The mean $v_{\text {mac }}$, excluding the three outliers with $v_{\text {mac }}$ greater than $20 \mathrm{~km} \mathrm{~s}^{-1}$, equals $12.1 \mathrm{~km} \mathrm{~s}^{-1}\left(\sigma=4.2 \mathrm{~km} \mathrm{~s}^{-1}, 40 \mathrm{RR}\right.$ Lyr stars $)$, and the corresponding mean $v \sin i$ (after eliminating the three $v \sin i$ outliers) is $4.3 \mathrm{~km} \mathrm{~s}^{-1}$ ( $\sigma=1.2 \mathrm{~km} \mathrm{~s}^{-1}, 40 \mathrm{RR}$ Lyr stars). The surface gravity versus metallicity diagram (lower right panel) reveals a clear trend between $\log g$ and $[\mathrm{M} / \mathrm{H}]$ : the metal-rich RR Lyrae stars have larger surface gravities (hence smaller luminosities) than the more metal-poor RR Lyrae stars, which was explained by Sandage's (1958) "stacked horizontal-branch luminosity levels" model (see Bono et al. 1997, and discussion in N11). No significant differences between the Blazhko and nonBlazhko stars are evident in any of the diagrams of Figure 10.

\section{PHOTOMETRIC METAL ABUNDANCES FROM $P-\phi_{31}-[\mathrm{Fe} / \mathrm{H}]$ RELATIONSHIPS}

Derivation of metal abundances from correlations relating pulsation period, spectroscopic $[\mathrm{Fe} / \mathrm{H}]$, and one or more parameters that characterize the shape of the photometric light curve (e.g., $\phi_{31}^{s}, A_{\text {tot }}$, risetime, etc.) was discussed in Section 3. In this section we use the accurate pulsation periods and Fourier light curve parameters presented in Section 3, and the spectroscopic metal abundances derived in Section 4, to derive new $P-\phi_{31}-[\mathrm{Fe} / \mathrm{H}]$ regressions for the Kepler-field RRab and RRc stars. Using our fitted models we estimate $[\mathrm{Fe} / \mathrm{H}]_{\text {phot }}$ for the program stars and investigate the applicability of the models to Blazhko and non-Blazhko stars. Because the spectroscopic $[\mathrm{Fe} / \mathrm{H}]$ values are derived from high dispersion spectra analyzed using standard reduction procedures, the derived metallicities are on the scale of the HDS (i.e., the Carretta et al. 2009, hereafter C9 scale).

\subsection{RRab Stars}

Several models were evaluated for the Kepler-field RRab stars and the optimum fit was achieved with the following nonlinear model:

$$
[\mathrm{Fe} / \mathrm{H}]=b_{0}+b_{1} P+b_{2} \phi_{31}^{s}+b_{3} \phi_{31}^{s} P+b_{4}\left(\phi_{31}^{s}\right)^{2},
$$

where $\phi_{31}^{s}$ is the mean $\phi_{31}^{s}(K p)$ value and $[\mathrm{Fe} / \mathrm{H}]$ is the VWA spectroscopic value (see Table 7). Excluded from the final calibration were five Blazhko stars with large residuals: V445 Lyr and V2178 Cyg, both of which exhibit extremely large amplitude and frequency modulations (see Table 2, and right side of Figure 3), and KIC 11125706, V838 Cyg, and V1104 Cyg, which have small amplitude and frequency modulations (see Table 2, Figures 2 and 3 and Section 3.2.1). Also excluded from the calibration were two non-Blazhko stars with unreliable metallicities, V346 Lyr and V1510 Cyg. The resulting estimated coefficients and their standard errors are: $b_{0}=-8.65 \pm 4.64$, $b_{1}=-40.12 \pm 5.18, b_{2}=5.96 \pm 1.72, b_{3}=6.27 \pm 0.96$, and $b_{4}=-0.72 \pm 0.17$. The rms error of the fit was $0.084 \mathrm{dex}$, with adjusted $R^{2}=0.98$. Seventeen of the 26 calibration stars were non-Blazhko variables and nine were Blazhko stars. The photometric $[\mathrm{Fe} / \mathrm{H}]$ values derived from the fitted model are given in Column 9 of Table 1, where the estimated standard errors were calculated using the usual Gaussian theory formula for nonlinear least squares regression (analogous to Equations (4) and (5) of JK96, except that we have ignored the errors in $\phi_{31}^{s}$ and $P$ owing to the high accuracy of the Kepler photometry).

In Figure 11 the fitted photometric metallicities are plotted against the spectroscopic metallicities (top panel). Also shown (bottom panel) are the differences, $\Delta[\mathrm{Fe} / \mathrm{H}]=[\mathrm{Fe} / \mathrm{H}]_{\text {phot }}-$ $[\mathrm{Fe} / \mathrm{H}]_{\text {spec }}$, plotted against $[\mathrm{Fe} / \mathrm{H}]_{\text {spec }}$. Included in the graphs are the five Blazhko stars that were excluded from the final calibration (labeled in the bottom panel). All of the calibrating stars lie close to the $1: 1$ line, with $|\Delta[\mathrm{Fe} / \mathrm{H}]|<0.13$ dex for all 17 non-Blazhko stars.

The largest metallicity differences correspond to the five Blazhko stars excluded from the calibration. It is perhaps not surprising that the two most extreme Blazhko stars (V445 Lyr, V2178 Cyg) showed the greatest disagreement. Since both stars exhibit considerable variation in $\phi_{31}^{s}$ over a Blazhko cycle, and since the calibration model (Equation (2)) is nonlinear, it is questionable (Jensen's inequality) whether substitution of mean $\phi_{31}$ rather than averaging the "instantaneous" $[\mathrm{Fe} / \mathrm{H}]$ values is the appropriate way of calculating $[\mathrm{Fe} / \mathrm{H}]_{\text {phot }}$. The $[\mathrm{Fe} / \mathrm{H}]_{\text {phot }}$ 

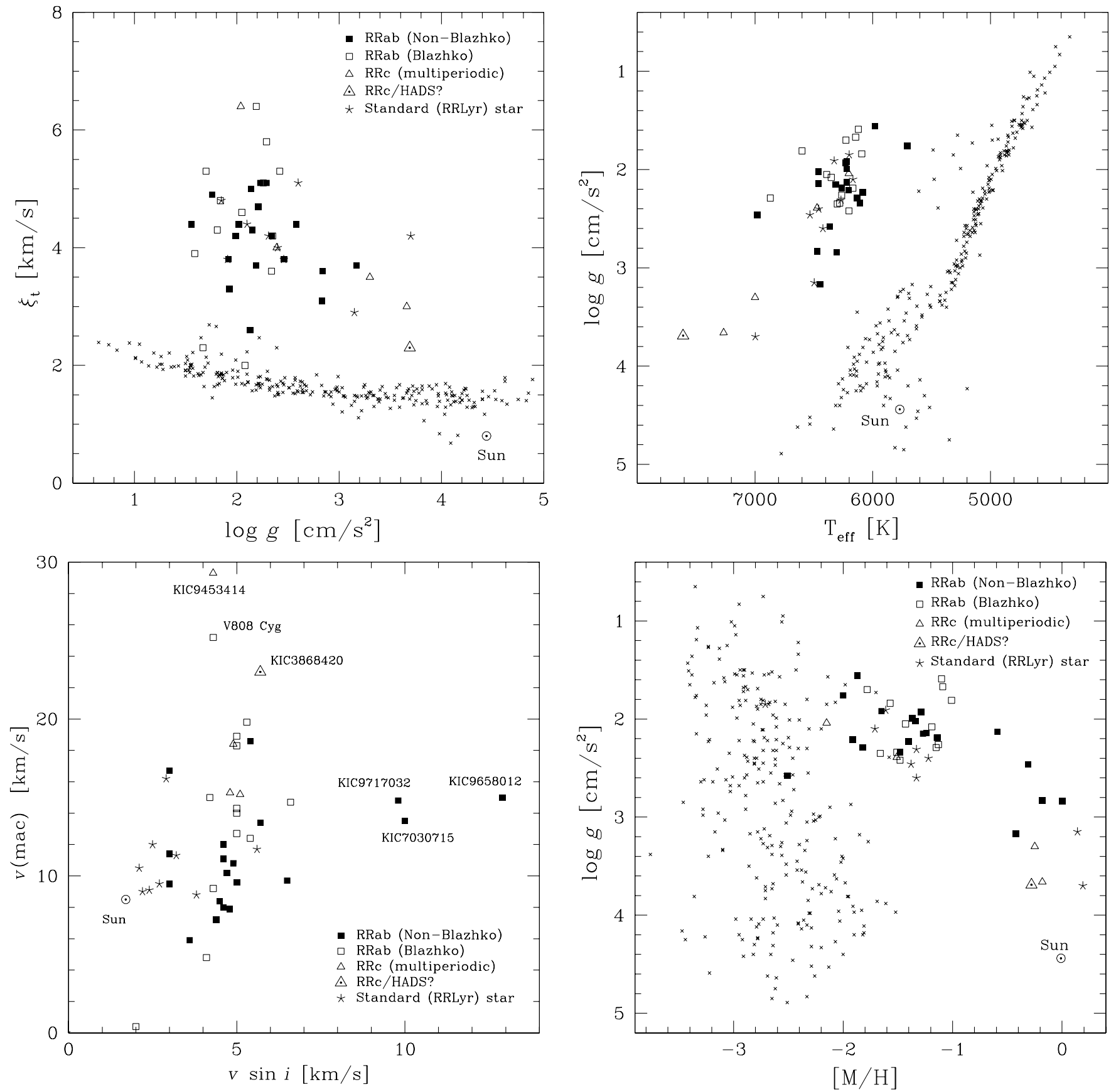

Figure 10. Four diagrams comparing the atmospheric parameters for the Kepler-field RR Lyrae stars, the nine RR Lyrae standards, and the Sun (all derived with SME), with the same parameters (also derived with SME) for the HERES metal-poor halo red giants and red horizontal branch stars (from Barklem et al. 2005). Upper left: microturbulent velocity, $\xi_{t}\left(\mathrm{~km} \mathrm{~s}^{-1}\right)$, vs. surface gravity, $\log g\left(\mathrm{~cm} \mathrm{~s}^{-2}\right)$ - the HERES stars are plotted as small crosses. Upper right: surface gravity, log $g$ $\left(\mathrm{cm} \mathrm{s}^{-2}\right)$, vs. effective temperature, $T_{\text {eff }}(\mathrm{K})$-here the HERES stars clearly are seen to be mainly red giants, subgiants, and $\sim 10$ red horizontal branch stars. Lower left: macroturbulent velocity, $v_{\text {mac }}$, vs. projected rotation velocity, $v \sin i\left(\mathrm{~km} \mathrm{~s}^{-1}\right)$, with labels identifying the most extreme stars $\left(v_{\operatorname{mac}}\right.$ and $v \sin i$ were not given for the HERES stars). Lower right: surface gravity, $\log g\left(\mathrm{~cm} \mathrm{~s}^{-2}\right)$, vs. metal abundance, $[\mathrm{M} / \mathrm{H}]$.

given in Table 1 were calculated using the first (i.e., substitution of mean- $\left.\phi_{31}^{s}\right)$ method. To investigate the second method we computed $[\mathrm{Fe} / \mathrm{H}]_{\text {phot }}$ for the five Blazhko stars by averaging (over complete Blazhko cycles) the metal abundances evaluated (Equation (2)) at each "instant" along the $\phi_{31}^{s}$ time series. As expected, the two methods gave similar results for the three low amplitude/frequency modulators. However, for V445 Lyr and V2178 Cyg averaging over the instantaneous abundances gave $[\mathrm{Fe} / \mathrm{H}]_{\text {phot }}=-1.69$ and -1.65 dex, respectively, both of which are much closer to the spectroscopic abundances: -1.78 dex for V445 Lyr (which is from the SME analysis since the VWA analysis was inconclusive), and $-1.66 \pm 0.13$ dex for V2178 Cyg.

Of course the spectroscopic metallicities are also uncertain and contribute to the differences. Four of the five Blazhko stars seem to have reliable (and consistent) metal abundances from the VWA and SME analyses. The largest difference, that for V445 Lyr (Figure 11), was calculated assuming the SME abundance, -1.78 dex (which appears to be consistent with the partial spectrum shown in Figure 7(a)). Consideration of the pulsation phases at the times of the spectroscopic observations, and of the Blazhko properties of the stars, reveals no obvious patterns: 

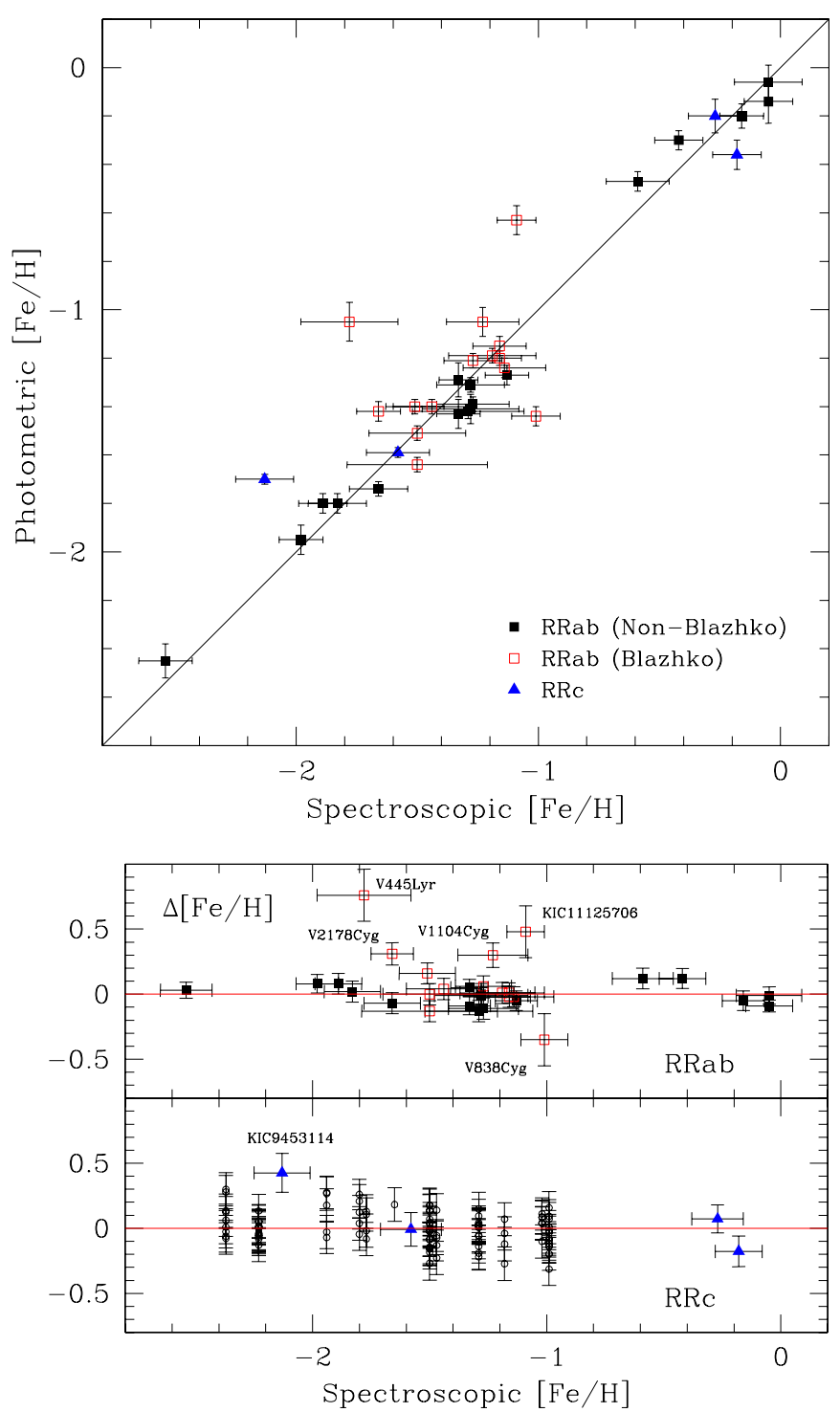

Figure 11. Comparison of the spectroscopic and photometric metallicities for the Kepler-field RR Lyrae stars. The diagonal line in the top panel is the $1: 1$ relation. The bottom panel contains the $[\mathrm{Fe} / \mathrm{H}]$ differences (photometric minus spectroscopic) for the RRab (upper) and RRc (lower) stars, where the vertical error bars are the standard errors of the differences. Included in the lowest graph are the residuals for the M07 globular cluster RRc calibration data set (small open circles).

(A color version of this figure is available in the online journal.)

the pulsation phases range from 0.31 to 0.43 ; four of the five stars have Blazhko periods 50 days, while V2178 Cyg has a considerably longer $P_{\mathrm{BL}}=234 \pm 10$ days; and the Blazhko phases range from 0.32 to 0.82 .

We conclude that since the large metallicity differences found for extreme Blazhko stars are reduced when "instantaneous" $[\mathrm{Fe} / \mathrm{H}]_{\text {phot }}$ values are averaged instead of calculated using the mean- $\phi_{31}$ method, and that the two methods give the same result for the non-Blazhko and less extreme Blazhko stars, that this method might be preferable for future $[\mathrm{Fe} / \mathrm{H}]_{\text {phot }}$ calculations.

In Figure 12 two sets of isometallicity curves have been added to the $P-\phi_{31}$ diagram (see Figure 4$)$. The solid curves (black) were calculated by solving our nonlinear relation (Equation (2)) for five $[\mathrm{Fe} / \mathrm{H}]$ values (ranging from -2.0 to -0.1 dex), and the dashed lines (blue) were calculated using the well-known JK96 formula (their Equation (3)) and solving for $[\mathrm{Fe} / \mathrm{H}]=$
$-2.0,-1.0$ and 0.0 dex. Since our photometry is on the $K p$ system it was necessary to transform the JK96 formula to the $K p$ system, which was done using Equation (2) of N11, i.e., $\phi_{31}^{s}(V)=\phi_{31}^{s}(K p)-0.151( \pm 0.026)$, resulting in

$$
[\mathrm{Fe} / \mathrm{H}]=-5.241-5.394 P+1.345 \phi_{31}^{s}(K p) .
$$

The original version of this relation was established using a calibration data set consisting of 81 galactic-field RR Lyr stars with reliable $V$ light curves and HDS abundances. It was considered optimal after various linear and nonlinear models were investigated, and gives photometric abundances on the HDS metallicity scale established by Jurcsik (1995). It is applicable for stars meeting the compatibility condition, $D_{m}<3$, a criterion that fails to hold for stars with peculiar light curves, such as certain Blazhko variables and RR Lyr stars in advanced evolutionary stages.

In general our nonlinear model and the JK96 model agree for $[\mathrm{Fe} / \mathrm{H}]<-1.0$ dex, with progressively larger discrepancies occurring as $[\mathrm{Fe} / \mathrm{H}]$ decreases. It is well-known that for the lowest $[\mathrm{Fe} / \mathrm{H}]$ stars the JK96 formula gives metallicities too metal-rich by $\sim 0.3 \mathrm{dex}$, the problem having been identified by JK96 themselves and discussed further by Nemec (2004) and Smolec (2005). Our model appears to correct this problem (compare the $[\mathrm{Fe} / \mathrm{H}]=-2.0$ dex curves in Figure 12), in part due to the wider inclusion in our sample of stars more metal-poor than $-2.0 \mathrm{dex}$, as well as our use of a nonlinear rather than linear model. It should also be noted that JK96 adopted a spectroscopic $[\mathrm{Fe} / \mathrm{H}]$ of -2.10 dex for X Ari, whereas the spectroscopic metallicities in Table 10 suggest a much lower value, $[\mathrm{Fe} / \mathrm{H}] \sim-2.65 \mathrm{dex}$.

The Kepler-field RR Lyr stars divide into two groups, those with $\phi_{31}^{s}(K p) \sim 5$ having low metallicities, $[\mathrm{Fe} / \mathrm{H}]<-1$ dex, and a high metallicity group with $\phi_{31}^{s}(K p) \sim 5.7$. Four of the five Kepler stars comprising the latter group were identified as metal-rich by N11 (V782 Cyg, V784 Cyg, KIC 6100702 and V2470 Cyg); to this group we can now add V839 Cyg (and the Keck standard stars V445 Oph and SW And).

\subsection{RRc Stars}

The $P-\phi_{31}-[\mathrm{Fe} / \mathrm{H}]$ relation for RRc stars was investigated previously by M07. Two nonlinear formulas were derived, one for metallicities on the ZW84 scale (Zinn \& West 1984) and the other for the CG97 scale (Carretta \& Gratton 1997). For calibration purposes 106 stars in 12 galactic globular clusters were used, with cluster mean metallicities ranging from -2.2 to -1.0 dex

With only four RRc stars in our sample it was not possible to derive independently a similar relation for the Kepler-field RRc stars. We therefore added the Kepler stars to the M07 calibration data set (kindly sent to us by Dr. Morgan) and derived a new relation. The inclusion of the Kepler stars extends the $[\mathrm{Fe} / \mathrm{H}]$ range to considerably higher metallicities.

Since most of the data were from the M07 sample we chose to work with $\phi_{31}^{c}(V)$ Fourier phase parameters. The $\phi_{31}^{s}(K p)$ values for the Kepler stars were transformed to $\phi_{31}^{c}(V)$ values using Equation (2) of N11, which, while probably not optimal for the hotter RRc stars (since it was derived for non-Blazhko $\mathrm{RRab}$ stars) is all that is presently available; the sine-to-cosine transformation was made using $\phi_{31}^{c}=\phi_{31}^{s}-\pi$.

The metallicities assumed for the Kepler stars were those given in Table 7 , and the $[\mathrm{Fe} / \mathrm{H}]$ values assumed for the globular cluster stars were the cluster means given in Table A.1 of C9. 


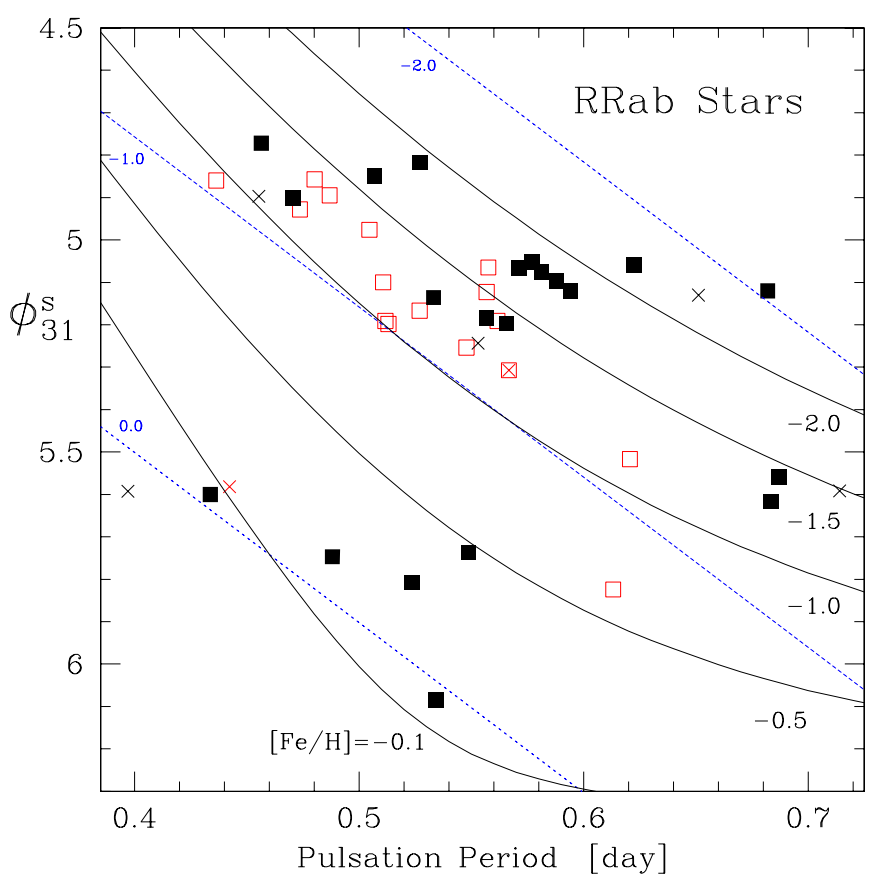

Figure 12. Period- $\phi_{31}$ diagram for the Kepler-field RRab stars. Also shown are five isometallicity curves (black solid curves) derived from our nonlinear $P-\phi_{31}^{S}-[\mathrm{Fe} / \mathrm{H}]$ relation (Equation (2)), and three isometallicity curves (blue dotted lines) derived from the JK96 linear relation. The Kepler-field nonBlazhko RRab stars (black filled squares) and Blazhko RRab stars (red open squares) are also plotted, along with six Keck spectroscopic standard stars (crosses). The $\phi_{31}^{s}$ values were derived from sine-series Fourier decomposition of the $K p$-passband observations.

(A color version of this figure is available in the online journal.)

The latter are very similar to the metal abundances adopted by W. Harris in the latest edition (2010) of his online catalog of globular cluster parameters (Harris 1996).

Using the revised data set and the $\phi_{31}^{c}(V)$ phase parameters we refit the M07 model,

$$
[\mathrm{Fe} / \mathrm{H}]=b_{0}+b_{1} P+b_{2} \phi_{31}^{c}+b_{3} \phi_{31}^{c} P+b_{4} P^{2}+b_{5}\left(\phi_{31}^{c}\right)^{2}
$$

where the metallicities are now on the $\mathrm{C} 9$ scale. After removing nine outliers (V17 in M9; V26 in NGC 6934; V27 and V19 in M2; V50 in M15; V3, V11, and V14 in NGC 4147; and KIC 9453114) the estimated model coefficients and their standard errors were found to be: $b_{0}=1.70 \pm 0.82, b_{1}=$ $-15.67 \pm 5.38, b_{2}=0.20 \pm 0.21, b_{3}=-2.41 \pm 0.62$, $b_{4}=18.00 \pm 8.70$, and $b_{5}=0.17 \pm 0.04$. The rms error of the fit was $0.13 \mathrm{dex}$, the adjusted $R^{2}=0.94$, and the calibration sample size $N=101$ stars. The fitted $[\mathrm{Fe} / \mathrm{H}]_{\text {phot }}$ (and their standard errors) for the Kepler-field RRc stars are given in Column 9 of Table 1.

The metallicity differences for the four Kepler-field RR Lyr stars (blue triangles) and for the globular cluster stars (small open circles) are plotted in the bottom panel of Figure 11. For the three Kepler stars that were included in the calibration the differences are small, all under 0.18 dex; for KIC 9453114 the derived $[\mathrm{Fe} / \mathrm{H}]_{\text {phot }}$ is 0.43 dex more metal rich than the $[\mathrm{Fe} / \mathrm{H}]_{\text {spec }},-2.13 \pm 0.12$ dex. For the cluster stars the groups correspond to the individual clusters, with the vertical variation reflecting the range of the $P$ and $\phi_{31}$ values within each cluster (for clarity the uncertainties in the $[\mathrm{Fe} / \mathrm{H}]_{\text {spec }}$ have been omitted).

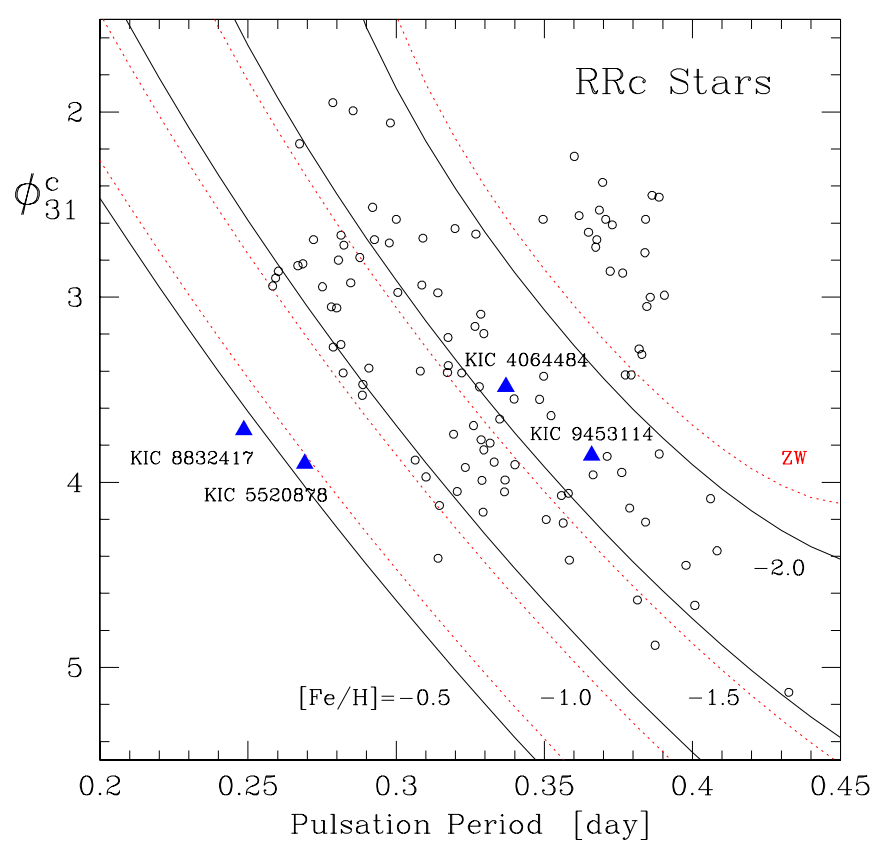

Figure 13. Period- $\phi_{31}$ diagram for RRc stars. The Kepler-field RRc stars (blue triangles) have been labeled with KIC number. Also plotted are the 106 Galactic globular cluster RRc stars that were used by M07 in the derivation of their $P-\phi_{31}^{c}-[\mathrm{Fe} / \mathrm{H}]$ relations (small open circles), and two sets of isometallicity curves (the black curves are for the $\mathrm{C} 9$ scale, and the red dotted curve for the ZW84 scale). The $\phi_{31}^{c}$ values were derived from cosine Fourier decomposition of $V$-passband observations.

(A color version of this figure is available in the online journal.)

In Figure 13 the $P-\phi_{31}^{c}$ diagram is plotted for the RRc stars in the Kepler-field and in the M07 globular clusters (the symbols are the same as in Figure 11). Also shown are two sets of isometallicity curves for $[\mathrm{Fe} / \mathrm{H}]$ ranging from -2.0 dex to -0.5 dex. The solid curves (black) were calculated using the new $P-\phi_{31}^{c}(V)-[\mathrm{Fe} / \mathrm{H}]$ relation (Equation (4)) and solving for the four different $[\mathrm{Fe} / \mathrm{H}]$ values; and the dotted curves (red) are the same curves transformed (using the transformation equation given in C9) to the ZW84 scale.

\section{SUMMARY}

Metal abundances, RVs and atmospheric properties have been derived for 41 RR Lyr stars located in the field of view of the Kepler space telescope. The spectroscopic $[\mathrm{Fe} / \mathrm{H}]$ values range from $-2.54 \pm 0.13$ (NR Lyr) to $-0.05 \pm 0.13$ dex (V784 Cyg). Four stars that were suspected by N11 from Q0-Q5 Kepler photometry of being metal rich (KIC 6100702, V2470 Cyg, V782 Cyg, and V784 Cyg) are here confirmed as such, and three more metal-rich RR Lyr stars are identified: V839 Cyg, KIC 5520878, and KIC 8832417 (the last two being RRc stars).

For all but five Blazhko RRab stars and one RRc star the $[\mathrm{Fe} / \mathrm{H}]_{\text {spec }}$ are in good agreement with newly calculated $[\mathrm{Fe} / \mathrm{H}]_{\text {phot }}$ values derived from detailed analyses of $\sim 970$ days of quasi-continuous high-precision Q0-Q11 LC and SC Kepler photometry. Revised pulsation periods and times of maximum light are given for all the stars (these were needed for calculating the pulsation phases at the times of the spectroscopic observations and the $[\mathrm{Fe} / \mathrm{H}]_{\text {phot }}$ values), and updated Blazhko periods and times of maximum amplitude are given for the amplitude modulated variables (needed for calculating Blazhko phases).

We conclude that empirical Fourier-based $P-\phi_{31}-[\mathrm{Fe} / \mathrm{H}]$ relations can be used to derive $[\mathrm{Fe} / \mathrm{H}]_{\text {phot }}$ for non-Blazhko and 
most Blazhko RRab stars (provided that the modulation is not too extreme and that sufficient Blazhko cycles are used when calculating the average $\phi_{31}$ ); similar conclusions previously were reached by Smolec (2005) for Blazhko and non-Blazhko stars in the LMC and Galactic bulge (OGLE survey), and by Jurcsik et al. (2012) for Galactic field stars observed by the Konkoly Blazhko Survey. For three of the four RRc stars the $[\mathrm{Fe} / \mathrm{H}]_{\text {phot }}$ calculated from empirical Fourier relations are in good agreement with the measured $[\mathrm{Fe} / \mathrm{H}]_{\text {spec }}$ values. We also suggest that the use of "instantaneous" $[\mathrm{Fe} / \mathrm{H}]_{\text {phot }}$ values might be preferable to the usual method of calculating $[\mathrm{Fe} / \mathrm{H}]_{\mathrm{phot}}$ using mean- $\phi_{31}$ values.

All the Blazhko stars are found to exhibit both amplitude and frequency modulations, and five of the 21 non-Blazhko RRab stars are more metal-rich than $[\mathrm{Fe} / \mathrm{H}] \sim-1.0$ dex while none of the 16 Blazhko stars is more metal-rich than this. Several stars are found to have special photometric characteristics.

1. V838 Cyg, classified originally as a non-Blazhko star (B10; N11), has been discovered here to be a Blazhko star with a complex power spectrum and the smallest amplitude modulation yet detected in such a star, $\Delta A_{1}=$ 0.0024 mag - it joins KIC 11125706 as the current record holders.

2. V2178 Cyg is an extreme Blazhko star with a unique up-down pattern seen in the time variation of its Fourier $\phi_{31}$ parameter (Figure 4).

3. V354 Lyr has a Blazhko period of just under $2 \mathrm{yr}$, the longest $P_{\mathrm{BL}}$ of any Kepler Blazhko star.

4. The candidate RRc star KIC 3868420 is shown to have a high metallicity, a high surface gravity, a high $v_{\text {mac }}$, and a low RV; while its atmospheric characteristics are not unlike those of the two metal-rich RRc stars in the Kepler field, we conclude based on the shape of its light curve and its rather short period that it more probably is a HADS star than an RRc star.

5. V349 Lyr (KIC 7176080) has been classified previously as a Blazhko star with an amplitude modulation period greater than 127 days, and as a non-Blazhko star; analysis of the currently available data (Q1-Q13) supports the latter classification.

Finally, atmospheric parameters are derived for the stars, from which we conclude that the RR Lyrae stars have higher microturbulent velocities (and are hotter) than the RHB and metal-poor red giants of the same surface gravity. The observations also directly confirm that the more metal-rich RR Lyrae stars have higher surface gravities than the more metal poor RR Lyrae stars.

The CFHT and Keck spectra contain much more chemical information than the $[\mathrm{Fe} / \mathrm{H}]$ values and atmospheric parameters presented here. Of particular interest are relative abundance ratios $[\mathrm{X} / \mathrm{Fe}]$, especially $[\alpha / \mathrm{Fe}]$ ratios which are related to the supernovae history of the Galaxy. The expectation is that the $\alpha$-elements $(\mathrm{O}, \mathrm{Ne}, \mathrm{Mg}, \mathrm{Si}, \mathrm{S}, \mathrm{Ca}, \mathrm{Ti})$ produced mainly by Type II supernovae, will be enhanced with respect to Fe (produced mainly by Type Ia supernovae) by $\sim 0.4$ for the stars more metal poor than $[\mathrm{Fe} / \mathrm{H}]=-0.8 \mathrm{dex}$ (see C95). Such measurements will be presented elsewhere.

Funding for the Kepler Discovery mission is provided by NASA's Science Mission Directorate. The authors thank the entire Kepler team without whom these results would not be possible. We thank Nadine Mansett and her team of service observers at the Canada-France-Hawaii $3.6 \mathrm{~m}$ telescope for successfully acquiring the CFHT spectra. J.N. would like to thank Jozsef Benkő, Johanna Jurcsik, Lászlo Molnár and Róbert Szabó for discussions on Blazhko stars and for their hospitality at KASC5 and in Budapest. He also greatly appreciates discussions with Katrien Kolenberg regarding her RR Lyrae work, with Robert Stellingwerf concerning his PDM2 program, with BiQing for concerning the results of her PhD thesis, with Andrzej Pigulski for discussions about the ASAS-North survey. John Feldmeier sent information on the two BOKS survey RR Lyr stars, Géza Kovács kindly provided J.N. with his Fourier decomposition software, and Siobahn Morgan sent her file of Fourier parameters for RRc stars in galactic globular clusters. Finally, special thanks go to Amanda Linnell Nemec, Young-Beom Jeon, and Robert Szabó for their helpful comments on the manuscript, and to George Preston for his useful referee report. J.N. acknowledges support from the Camosun College Faculty Association; J.C. and B.S. acknowledge NSF grant AST-0908139; and P.M. is supported by Polish NCN grant DEC-2012/05/B/ST9/03932. This project has been supported by the Hungarian OTKA Grants K76816, K83790 an MB08C 81013 and the "Lendület-2009" Young Researchers Program of the Hungarian Academy of Sciences. A.D. was supported by the Hungarian Eötvös fellowship and by the János Bolyai Research Scholarship of the Hungarian Academy of Sciences. Finally, all the authors wish to recognize and acknowledge the very significant cultural role and reverence that the summit of Mauna Kea has always had within the indigenous Hawaiian community; we are most fortunate to have had the opportunity to conduct observations from this mountain.

Facilities: CFHT, Keck:I, Kepler

\section{REFERENCES}

Alcock, C., Allsman, R., Alves, D. R., et al. 1998, ApJ, 492, 190 Alcock, C., Allsman, R., Alves, D. R., et al. 2000, ApJ, 542, 257 Alcock, C., Alves, D. R., Axelrod, T. S., et al. 2004, AJ, 127, 334 Alcock, C., Alves, D. R., Becker, A., et al. 2003, ApJ, 598, 597 Arp, H. C. 1955 , AJ, 60, 317

Asplund, M., Grevesse, N., Sauval, A. J., \& Scott, P. 2009, ARA\&A, 47, 481 Balona, L. A., \& Nemec, J. M. 2012, MNRAS, 426, 2413

Barklem, P. S., Christlieb, N., Beers, T. C., et al. 2005, A\&A, 439, 129 Benedict, G. F., McArthur, B. E., Feast, M. W., et al. 2011, AJ, 142, 187 Benkő, J. M., Kolenberg, K., Szabó, R., et al. 2010, MNRAS, 409, 1585 (B10) Blazhko, S. 1907, AN, 175, 325

Bono, G., Caputo, F., Cassisi, S., et al. 1997, ApJ, 483, 811

Bono, G., Caputo, F., \& DiCriscienzo, M. 2007, A\&A, 476, 779

Brown, T. M., Latham, D. W., Everett, M. E., \& Esquerdo, G. A. 2011, AJ, 142,112

Bruntt, H., Bedding, T., Quirion, P.-O., et al. 2010a, MNRAS, 405, 1907

Bruntt, H., Catala, C., Garrido, R., et al. 2002, A\&A, 389, 345

Bruntt, H., De Cat, P., \& Aerts, C. 2008, A\&A, 478, 487

Bruntt, H., Deleuil, M., Fridlund, M., et al. 2010b, A\&A, 519, A51

Buchler, J. R., \& Kolláth, Z. 2011, ApJ, 731, 24

Buchler, J. R., \& Moskalik, P. 1992, ApJ, 391, 736

Cacciari, C., Corwin, T. M., \& Carney, B. 2005, AJ, 129, 267

Caputo, F., Castellani, V., Marconi, M., \& Ripepi, V. 2000, MNRAS, 316, 819 Carney, B. W., \& Jones, R. 1983, PASP, 95, 246

Carretta, E., Bragaglia, A., Gratton, R., D’Orazi, V., \& Lucatello, S. 2009, A\&A, 508, 695 (C9)

Carretta, E., \& Gratton, R. G. 1997, A\&AS, 121, 95 (CG97)

Cassisi, S., Castellani, V., degl'Innocenti, S., Salaris, M., \& Weiss, A. 1999, A\&AS, 134, 103

Clement, C., Muzzin, A., Dufton, Q., et al. 2001, AJ, 122, 2587

Clementini, G., Carretta, E., Gratton, R., et al. 1995, AJ, 110, 2319 (C95)

Cohen, J. G. 2011, ApJL, 7430, L38

Cohen, J. G., \& Huang, W. 2009, ApJ, 701, 1053

Cohen, J. G., \& Melendez, J. 2005a, AJ, 129, 303

Cohen, J. G., \& Melendez, J. 2005b, AJ, 129, 1607

Collinge, M. J., Sumi, T., \& Fabrycky, E. 2006, ApJ, 651, 197 
Donati, J.-F., Semel, M., Carter, B. D., Rees, D. E., \& Collier Cameron, A. 1997, MNRAS, 291, 658

Drukier, G. A., Cohn, H. N., Lugger, P. M., et al. 2007, AJ, 133, 1041

Feast, M. W., Laney, C. D., Kinman, T. D., van Leeuwen, F., \& Whitelock, P. A. 2008, MNRAS, 386, 2115

Feldmeier, J., Howell, S. B., Sherry, W., et al. 2011, AJ, 142, 2

Fernley, J., Barnes, T. G., Skillen, I., et al. 1998, A\&A, 330, 515

For, B.-Q., Sneden, C., \& Preston, G. W. 2011, ApJS, 197, 29

Garofalo, A., Cusano, F., Clementini, G., et al. 2013, ApJ, 767, 62

Gilliland, R. L., Jenkins, J. M., Borucki, W. J., et al. 2010, ApJL, 713, L160

Gratton, R., Bragaglia, A., Clementini, G., et al. 2004, A\&A, 421, 937

Grevesse, N., Asplund, M., \& Sauval, A. J. 2007, SSRv, 130, 105

Guggenberger, E., Kolenberg, K., Nemec, J. M., et al. 2012, MNRAS, 424, 649 (G12)

Gustafsson, B., Bell, R. A., Eriksson, K., \& Nordlund, A. 1975, A\&A, 42, 407

Gustafsson, B., Edvardsson, B., Eriksson, K., et al. 2008, A\&A, 486, 951

Harris, W. E. 1996, AJ, 112, 1487

Haschke, R., Grebel, E. K., Frebel, A., et al. 2012, AJ, 144, 88

Heiter, U., \& Eriksson, K. 2006, A\&A, 452, 1039

Heiter, U., Kupka, F., van't Veer-Menneret, C., et al. 2002, A\&A, 392, 619

Hinkle, K., Wallace, L., Valenti, J., \& Harmer, D. (ed.) 2000, Visible and Near-IR Atlas of the Arcturus Spectrum 3727-9300 ̊ (San Francisco, CA: ASP)

Holweger, H. 2001, in AIP Conf. Proc. 598, Solar and Galactic Composition: A Joint $\mathrm{SOHO} / A C E$ Workshop (Melville, NY: AIP), 23

Jenkins, J. M., Caldwell, D. A., Chandrasekaran, H., et al. 2010, ApJL, 713, L87

Jurcsik, J. 1995, AcA, 45, 653

Jurcsik, J., Hurta, Zs., Sódor, Á., et al. 2009a, MNRAS, 397, 350

Jurcsik, J., \& Kovács, G. 1996, A\&A, 312, 111 (JK96)

Jurcsik, J., Sódor, Á., Hajdu, G., et al. 2012, MNRAS, 423, 993

Jurcsik, J., Sódor, Á., Szeidl, B., et al. 2009b, MNRAS, 393, 1553

Jurcsik, J., Sódor, Á., Szeidl, B., et al. 2009c, MNRAS, 400, 1006

Jurcsik, J., Sódor, Á., Váradi, M., et al. 2005, A\&A, 430, 1049

Jurcsik, J., Szeidl, B., Sódor, Á., et al. 2006, AJ, 132, 61

Koch, D. G., Borucki, W. J., Basri, G., et al. 2010, ApJL, 713, L79

Kolenberg, K. 2002, PhD thesis, Katholieke Universiteit Leuven

Kolenberg, K., Bryson, S., Szabó, R., et al. 2011, MNRAS, 411, 878 (K11)

Kolenberg, K., Szabó, R., Kurtz, D. W., et al. 2010, ApJL, 713, L198 (K10)

Kolláth, Z., Molnár, L., \& Szabó, R. 2011, MNRAS, 414, 1111

Kovács, G. 1998, in ASP Conf. Ser. 135, A Half Century of Stellar Pulsation Interpretation: A Tribute to Arthur N. Cox, ed. P. A. Bradley \& J. A. Guzik (San Francisco, CA: ASP), 52

Kovács, G. 2005, A\&A, 438, 227

Kovács, G. 2009, in AIP Conf. Proc. 1170, Stellar Pulsation: Challenges for Theory and Observation, ed. J. A. Guzik \& P. A. Bradley (Melville, NY: AIP), 261

Kovács, G., \& Jurcsik, J. 1996, ApJL, 466, L17

Kovács, G., \& Walker, A. R. 2001, A\&A, 371, 579

Kovács, G., \& Zsoldos, E. 1995, A\&A, 293, L57

Kunder, A., \& Chaboyer, B. 2008, AJ, 136, 2441

Kunder, A., \& Chaboyer, B. 2009, AJ, 138, 1284

Kupka, F., Piskunov, N. E., Ryabchikova, T. A., Stempels, H. C., \& Weiss, W. W. 1999, A\&AS, 138, 119

Kurucz, R. L., Furenlid, I., Brault, J., \& Testerman, L. 1984, Solar Flux Atlas from 296 to $1300 \mathrm{~nm}$ (Sunspot, New Mexico: National Solar Observatory)

Lambert, D. L., Heath, J. E., Lemke, M., \& Drake, J. 1996, ApJS, 103, 183 (L96)

Layden, A. C. 1994, AJ, 108, 1016 (L94)

Le Borgne, J.-F., Klotz, A., Poretti, E., et al. 2012, AJ, 144, 39

Lenz, P., \& Breger, M. 2005, CoAst, 146, 53

Liu, T., \& Janes, K. A. 1989, ApJS, 69, 593

Liu, T., \& Janes, K. A. 1990a, ApJ, 354, 273

Liu, T., \& Janes, K. A. 1990b, ApJ, 360, 561

Molnár, L., Kolláth, Z., Szabó, R., et al. 2012, ApJL, 757, L13

Morgan, S. M., Wahl, J. N., \& Wieckhorst, R. M. 2007, MNRAS, $374,1421(\mathrm{M} 07)$
Moskalik, P., \& Buchler, J. R. 1991, ApJ, 366, 300

Moskalik, P., \& Kołaczkowski, Z. 2009, MNRAS, 394, 1649

Moskalik, P., \& Poretti, E. 2003, A\&A, 398, 213

Moskalik, P., Smolec, R., Kolenberg, K., et al. 2012, arXiv:1208.4251v1

Nardetto, N., Fokin, A., Mourard, D., et al. 2004, A\&A, 428, 131

Nardetto, N., Gieren, W., Kervella, P., et al. 2009, A\&A, 502, 951

Nemec, J. M. 2004, AJ, 127, 2185

Nemec, J. M., Smolec, R., Benkő, J. M., et al. 2011, MNRAS, 417, 1022 (N11)

Nemec, J. M., Walker, A., \& Jeon, Y.-B. 2009, AJ, 138, 1310

Oke, J. B. 1966, ApJ, 145, 468

Oosterhoff, P. Th. 1939, Obs, 62, 104

Oosterhoff, P. Th. 1944, BAN, 10, 55

Pietrukowicz, P., Udalski, A., Soszyński, I., et al. 2012, ApJ, 750, 169

Piskunov, N. E. 1992, in Stellar Magnetism, ed. Yu. V. Glagolevskij \& I. I.

Romanyuk (St. Petersburg: Nauka), 92

Pojmanski, G. 2002, AcA, 52, 397

Preston, G. W. 1959, ApJ, 130, 507

Preston, G. W. 2009, RR Lyrae Atmospherics: Wrinkles Old and New, Henry Norris Russell Lecture, ftp://ftp.obs.carnegiescience.edu/pub/gwp/ HNRLecture

Preston, G. W. 2011, AJ, 141, 6

Rentzsch-Holm, I. 1996, A\&A, 312, 966

Rey, S.-C., Lee, Y.-W., Joo, J.-M., Walker, A., \& Baird, S. 2000, AJ, 119, 1824

Roederer, I. U., \& Sneden, C. 2011, AJ, 142, 22

Sandage, A. R. 1958, in Proc. Conf. at Vatican Observatory Stellar Populations, ed. D. J. O'Connell (Ricerche Astr. Specola Vaticana, Vol. 5; New York: Interscience), 41

Sandage, A. R. 1981, ApJ, 248, 161

Sandage, A. R. 1990, ApJ, 350, 603

Sandage, A. R. 2004, AJ, 128, 858

Sandage, A. R. 2010, ApJ, 722, 79

Schörck, T., Christlief, N., Cohen, J., et al. 2009, A\&A, 507, 817

Sesar, B. 2012, AJ, 144, 114

Sesar, B., Ivezic, Z., Grammer, S. H., et al. 2010, ApJ, 708, 717

Simon, N. R. 1985, ApJ, 299, 723

Simon, N. R. 1988, ApJ, 328, 747

Simon, N. R., \& Clement, C. 1993, ApJ, 410, 526

Simon, N. R., \& Lee, A. S. 1981, ApJ, 248, 291

Simon, N. R., \& Teays, T. J. 1982, ApJ, 261, 586

Smolec, R. 2005, AcA, 55, 59

Smolec, R., Soszynski, I., Moskalik, P., et al. 2012, MNRAS, 419, 2407

Sneden, C. 1973, PhD thesis, Univ. Texas

Sneden, C. 2002, Manual, MOOG: An LTE Stellar Line Analysis Program, http://www.as.utexas.edu/ chris/moog.html

Sneden, C., For, B.-Q., \& Preston, G. W. 2011, in RR Lyrae Stars, Metal-Poor Stars, and the Galaxy, ed. A. McWilliam (Carnegie Obs. Astrophys. Ser. 5; Pasadena, CA: Carnegie Observatories)

Soszynski, I., Dziembowski, W. A., Udalski, A., et al. 2011, AcA, 61, 1

Soszynski, I., Udalski, A., Szymański, M. K., et al. 2009, AcA, 59, 1

Soszynski, I., Udalski, A., Szymañski, M. K., et al. 2010, AcA, 60, 165

Sousa, S. G., Santos, N. C., Israelian, G., Mayor, M., \& Monteiro, M. J. P. F. G 2007, A\&A, 469, 783

Sousa, S. G., Santos, N. C., Mayor, M., et al. 2008, A\&A, 487, 373

Stellingwerf, R. 1978, ApJ, 224, 953

Suntzeff, N. B., Kraft, R. P., \& Kinman, T. D. 1994, ApJS, 93, 271

Szabó, R., Kolláth, Z., Molnár, L., et al. 2010, MNRAS, 409, 1244

Szeidl, B. 1988, in Multimode Stellar Pulsations, ed. G. Kovács, L. Szabados, \& B. Szeidl (Kultura, Budapest: Konkoly Observatory), 45

Szeidl, B., Jurcsik, J., Sódor, Á., Hajdu, G., \& Smitola, P. 2012, MNRAS, 424, 3094

Udalski, A., Olech, A., \& Szymanski, M. 1997, AcA, 47, 1

Valenti, J. A., \& Piskunov, N. 1996, A\&AS, 118, 595

Vogt, S. S., Allen, S. L., Bigelow, B. C., et al. 1994, Proc. SPIE, 2198, 362

Walker, A. R., \& Nemec, J. M. 1996, AJ, 112, 2026

Wallerstein, G., \& Huang, W. 2010, MmSAI, 81, 952

Wils, P., Lloyd, C., \& Bernhard, K. 2006, MNRAS, 368, 1757

Zinn, R., \& West, R. J. 1984, ApJS, 55, 45 (ZW84) 